\title{
Robust approach to thermal resummation: Standard Model meets a singlet
}

\author{
Philipp M. Schicho, ${ }^{a, b}$ Tuomas V.I. Tenkanen $^{b, c, d, e}$ and Juuso Österman ${ }^{a}$ \\ ${ }^{a}$ Department of Physics and Helsinki Institute of Physics, University of Helsinki, \\ P.O. Box 64, FI-00014 Helsinki, Finland \\ ${ }^{b} A E C$, Institute for Theoretical Physics, University of Bern, \\ Sidlerstrasse 5, CH-3012 Bern, Switzerland \\ ${ }^{c}$ Nordita, KTH Royal Institute of Technology and Stockholm University, \\ Roslagstullsbacken 23, SE-106 91 Stockholm, Sweden \\ ${ }^{d}$ Tsung-Dao Lee Institute \& School of Physics and Astronomy, Shanghai Jiao Tong University, \\ Shanghai 200240, China \\ e Shanghai Key Laboratory for Particle Physics and Cosmology, \\ Key Laboratory for Particle Astrophysics and Cosmology (MOE), Shanghai Jiao Tong University, \\ Shanghai 200240, China \\ E-mail: philipp.schicho@helsinki.fi, tuomas.tenkanen@su.se, \\ juuso.s.osterman@helsinki.fi
}

ABstract: Perturbation theory alone fails to describe thermodynamics of the electroweak phase transition. We review a technique combining perturbative and non-perturbative methods to overcome this challenge. Accordingly, the principal theme is a tutorial of hightemperature dimensional reduction. We present an explicit derivation with a real singlet scalar and compute the thermal effective potential at two-loop order. In particular, we detail the dimensional reduction for a real-singlet extended Standard Model. The resulting effective theory will impact future non-perturbative studies based on lattice simulations as well as purely perturbative investigations.

Keywords: Beyond Standard Model, Effective Field Theories

ArXiv EPRINT: 2102.11145 


\section{Contents}

1 Introduction 2

2 Thermal phase transitions 4

2.1 Down the pipeline 4

2.2 Dimensional reduction for a high-temperature 3d effective theory 6

$\begin{array}{lll}2.3 & \text { Approaches to thermodynamics of thermal phase transitions } & 7\end{array}$

$\begin{array}{lll}2.3 .1 & 4 \mathrm{~d} \text { approach } & 10\end{array}$

$\begin{array}{lll}\text { 2.3.2 Perturbative 3d approach } & 10\end{array}$

$\begin{array}{lll}2.3 .3 & \text { Non-perturbative 3d approach } & 11\end{array}$

3 Dimensional reduction with a real scalar: a tutorial $\quad 11$

$\begin{array}{ll}3.1 & \text { Model and parameter matching } \\ \end{array}$

$\begin{array}{ll}3.2 & \text { Computation of correlators } \\ & 15\end{array}$

3.2.1 Broken phase: correlators from the two-loop effective potential 15

3.2.2 Unbroken phase: correlators from the diagrammatic approach 18

3.2.3 Cancellation of mixed hard/soft terms 20

$\begin{array}{ll}3.3 & \text { Matching relations for 3d parameters }\end{array}$

3.4 Two-loop effective potential in 3d EFT 23

4 Dimensional reduction of the real-singlet extended Standard Model $\quad 24$

$\begin{array}{lll}4.1 & \text { Effective 3d theories } & 25\end{array}$

$\begin{array}{ll}4.2 & \text { Integrating out the hard scale }\end{array}$

$\begin{array}{lll}4.3 & \text { Integrating out the soft scale } & 33\end{array}$

5 Discussion $\quad 35$

$\begin{array}{ll}\text { A Detailed computation in the xSM } & 37\end{array}$

A.1 Counterterms and $\beta$-functions of the 4d theory 37

A.2 Correlators from the one-loop effective potential in general covariant gauge 39

A.3 Matching of marginal operators 40

A.4 Two-loop computation of correlators 42

$\begin{array}{ll}\text { B Collection of integrals } & 46\end{array}$ 


\section{Introduction}

A strong first-order cosmic phase transition (SFOPT) is a violent process that can trigger the generation of a primordial gravitational wave (GW) background (cf. [1, 2] and reviews [3-5]). Gravitational waves from astrophysical sources have been detected by Earth-based detectors LIGO and VIRGO for binary black hole [6-8] and neutron star mergers [9-11]. Their success and the mission to probe evidence of relic gravitational waves from the early Universe have sparked interest for space-based gravitational wave observatories such as LISA [12], BBO [13], Taiji [14], and DECIGO [15]. A detection of such a relic GW background could scope the underlying theories of particle physics complimentary to collider physics [16-19].

The electroweak phase transition (EWPT) is a smooth crossover in the minimal Higgs sector of the Standard Model (SM) [20-24]. Therein, the observed Higgs mass of $125 \mathrm{GeV}[25,26]$ exceeds the requirements for a SFOPT which precludes both the production of a cosmic GW background and electroweak baryogenesis [27]. The latter is a mechanism to produce the baryon asymmetry during the electroweak phase transition [28, 29]. New beyond the Standard Model (BSM) physics can alter the character of the electroweak symmetry breaking towards a SFOPT. To this end, new particles need to be sufficiently light in the vicinity of the electroweak (EW) scale and strongly enough coupled to the Higgs. This indicates that such BSM theories offer theoretical targets to guide future high-energy collider experiments [30].

One promising class of BSM candidates are theories with non-minimal Higgs sectors with distinctive collider phenomenology signatures; cf. refs. [17, 31-37]. These theories form a theoretical playground for the EWPT with ample related literature. The SM Higgs doublet can be accompanied for example by a singlet [38-58], second doublet [5968], triplet [69, 70], higher-order representations of SU(2) symmetry [71], combinations of these $[72,73]$ or higher dimensional operators [74-82]. Different models with non-minimal Higgs sector can accommodate SFOPT specifically but not exclusively at the EW scale around temperatures of $100 \mathrm{GeV}$. In addition, they could invoke sources for $\mathrm{CP}$ violation — the missing ingredient in the SM [83-89] required for electroweak baryogenesis - and potential dark matter candidates via new neutral scalars. Compellingly, a non-minimal Higgs sector can exhibit a rich pattern of phase transitions that progress in multiple consecutive steps [69, 90-93]. Phase transitions could have even occurred in a dark sector which couples to the SM only gravitationally. These transitions could potentially source a primordial GW background [94-101].

Thermal field theories are plagued by the infrared problem [102]. Their perturbative description of long distance modes is invalidated at high temperatures due to high occupancies of bosonic modes. Nevertheless, perturbation theory is still widely used when reorganising the perturbative expansion by resummation, such as in hard thermal loop perturbation theory [103] and daisy resummation [104].

A robust solution to the IR problem is achieved by an effective theory formulation of resummation. This allows to treat high-temperature heavy degrees of freedom perturbatively, while non-perturbative, light degrees of freedom are analysed with lattice gauge 
field theory techniques. Concretely, the phase transition thermodynamics is determined by Monte Carlo lattice simulations [105, 106] of dimensionally reduced high-temperature effective field theories (3d EFT). Originally established for non-Abelian gauge theories [107110], the formalism was generalised in the mid-1990s [111] and simultaneously successful in hot QCD [112-124] as reviewed in [125].

Considering the vastness of recent studies of the EWPT in BSM theories, the 3d EFT approach has been used scarcely. Seminal work in the 1990s for the Standard Model [106, 111] were continued in [21, 126-131], and also extended to SUSY models [132-142], the TwoHiggs Doublet Model (2HDM) [132, 143, 144], the Abelian Higgs Model [145-148], SU(5) GUT [149] and pure scalar field theory [150]. Recent studies reinvigorated the 3d approach for the SM accompanied by a real singlet (xSM) [151, 152], a real triplet ( $\Sigma \mathrm{SM})[153,154]$, the 2HDM [155-158], the SM with one simple higher dimensional operator [159] and a real scalar field theory [160].

Dimensional reduction implements the required resummations automatically upon perturbatively constructing the 3d EFT. Nonetheless, it is customary to study the EWPT in terms of the thermal effective potential $[161,162]$ computed directly with other resummation schemes [104, 163]. While improved two-loop computations exist [104, 164-166] (cf. also refs. $[167,168]$ ), it is typical for recent EWPT literature to implement a daisyresummed thermal effective potential only at one-loop level. However, the infrared problem persists and these fully perturbative studies of the EWPT are severely limited with their setbacks often underestimated. Even their qualitative description can - and often will fail. In contrast to lattice studies, transitions are often realised as (weak) first order since a crossover character is incompatible with perturbation theory.

Describing the EWPT thermodynamics all the way by a non-perturbative simulation poses a formidable task. This roots in analytical challenges related to the construction of required 3d EFTs and foremost excessive computational cost of simulations. Still, many perturbative studies of the EWPT could be significantly improved by employing perturbation theory within the dimensionally reduced 3d EFT. While this approach is entirely perturbative and hence incapable of solving the IR problem, it allows for systematic resummation and straightforward computations at two-loop level $[169,170]$. Thereby it supersedes the one-loop daisy-resummed thermal effective potential; see refs. [154, 158, 159] for recent direct comparisons. Indeed, this approach to perturbation theory was advocated already in ref. [137].

The dimensional reduction can be largely automated and the careful matching to multiple individual BSM theories streamlined. The task has been tackled recently $[159,171]$ and in this work at hand. As a consequence one can exploit the universality of the resulting dimensionally reduced EFTs to efficiently examine the parameter space of different BSM theories. This article combines these recent developments to extend previous work [151] for the $\mathrm{xSM}$ - a flagship model that is attractive for particle cosmology due to its minimal nature. Based on the construction of the $3 \mathrm{~d}$ EFT of the xSM, its applications $[172,173]$ chart a course of a state-of-the-art analysis of the EWPT thermodynamics. Thereby, perturbative scans that utilise a 3d EFT approach can guide non-perturbative simulations that finally solve the IR problem. 
This article is organised as follows. Section 2 reviews the computation of thermodynamics in generic scalar driven phase transitions, in particular focusing on the use of dimensionally reduced effective theories. Section 3 is a pedagogic tutorial to the construction of such a 3d EFT and computes the thermal effective potential at two-loop order, in the simplest case of a real singlet scalar. In section 4 , for the first time, we generalise the dimensional reduction to the xSM. Finally, section 5 discusses our results and outlook, while several technical details relevant to our computation are collected in the appendices.

\section{Thermal phase transitions}

The focus of this paper is to take steps towards a state-of-art determination of the cosmic phase transition thermodynamics for individual BSM theories with non-minimal Higgs sector. Direct ab-initio lattice calculations are, however, not feasible for a completely realistic $4 \mathrm{~d}$ description of the thermodynamics of electroweak phase transitions [174] due to problems related to chiral fermions. ${ }^{1}$ One alternative approach are non-perturbative simulations of dimensionally reduced effective theories. Following this idea, we survey the required technology on a generic level in the following section.

\subsection{Down the pipeline}

Several steps have to be considered to accurately predict gravitational waves from cosmological phase transitions. To this end, we illustrate a "pipeline" ranging from the collider phenomenology of BSM particle physics models to a primordial, stochastic gravitational wave background. Following a comprehensive ref. [176], we display different steps of this pipeline in figure 1 (ibid. ref. [176]).

From a theoretical standpoint, it is natural to start by defining the Lagrangian of the corresponding BSM theory. In our case of interest, the BSM field content enters as a nonminimal Higgs sector which contains one or more scalar fields. In general, the scalar fields can occur in any representation of the $\mathrm{SU}(2)$ symmetry and possess other symmetries and couplings to new gauge field or fermion content in a dark sector. However, several alternatives are conceivable (see refs. $[176,177]$ ), such as models for holographic phase transition [178-180] or Composite Higgs scenarios [181-183]. The pipeline constitutes the following steps:

Step (A): Relating collider signatures and BSM theory Lagrangian parameters. The Lagrangian (running) parameters are related to physical observables such as pole masses and mixing angles in zero-temperature perturbation theory. Then, actual collider signatures include production cross-sections and a relative shift in the Higgs couplings from their SM predicted values, ${ }^{2}$ and can constrain the available parameter space for

\footnotetext{
${ }^{1}$ However, $4 \mathrm{~d}$ simulations of purely bosonic theories are feasible to study; see ref. [175] and references therein.

${ }^{2}$ For a concrete example, in the case of xSM the proposed experimental signatures include the $h_{2} h_{2^{-}}$ production cross-section [33, 36, 49] and a modification to the $h_{1} Z Z$-coupling $[42,48,184]\left(h_{1}\right.$ and $h_{2}$ are 'mostly Higgs-like' and 'mostly singlet-like' scalar eigenstates).
} 


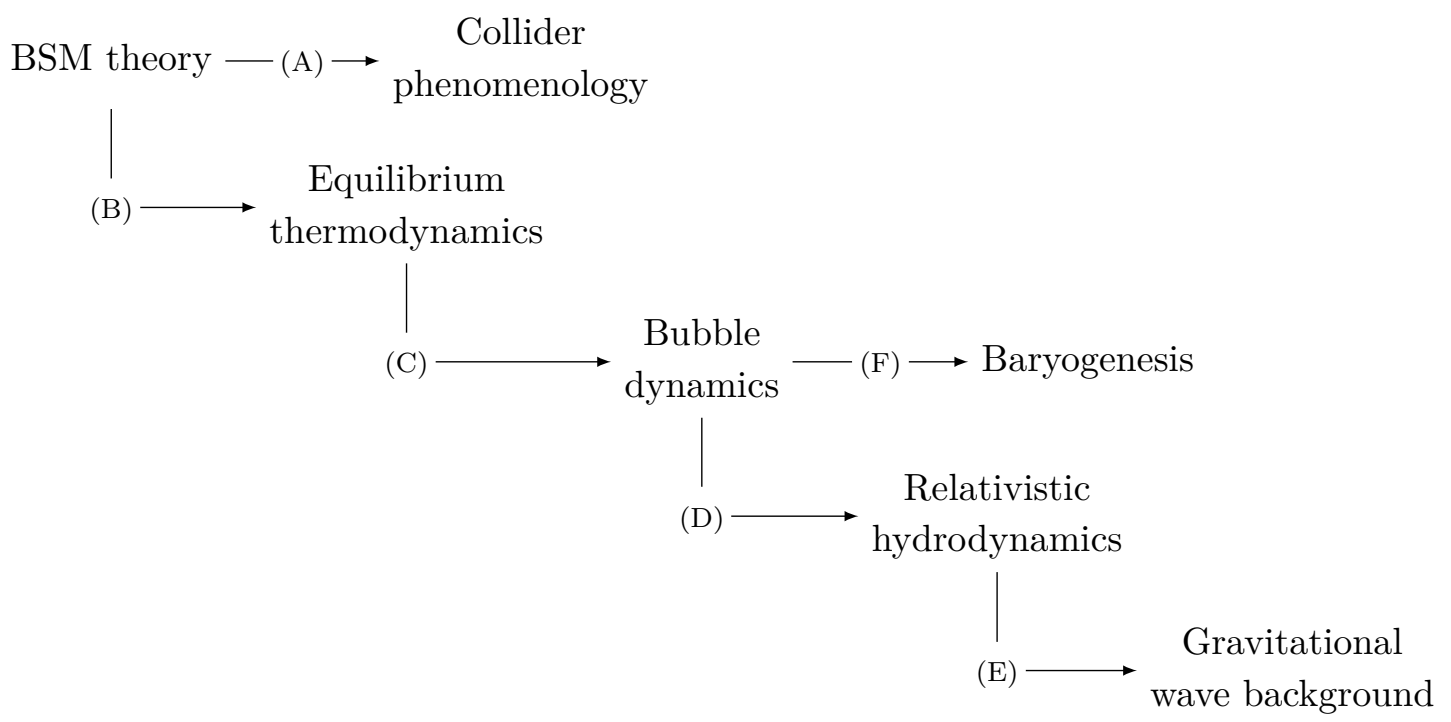

Figure 1. A pipeline between collider phenomenology of BSM theory and stochastic gravitational wave background signature. Later we focus on step (B), as even relatively small uncertainties in this step can propagate all the way down the pipeline and cause significant errors in the end.

phase transitions. The relation of the EWPT and collider physics is further discussed in ref. [30].

Step (B): Equilibrium thermodynamic properties as a function of BSM theory parameters. The former include the character of transition (crossover, first-order etc.), the critical temperature $\left(T_{\mathrm{c}}\right)$ and latent heat $\left(L / T_{\mathrm{c}}^{4}\right)$. They are encoded in the free energy of the system which is associated with the thermal effective potential in perturbation theory. Due to IR sensitivities at high- $T$, this step requires non-trivial resummations compared to perturbation theory at zero temperature, and eventually non-perturbative techniques. Step (B) is the main focus of the remaining sections of this article.

Step (C): If the phase transition is of first order, it proceeds by nucleation and expansion of bubbles of the broken phase in the presence of a surrounding plasma [185-188]. The bubble nucleation rate can be computed in a semi-classical approximation from the effective action which includes quantum and thermal corrections. The relevant quantities [189-193] are the Hubble parameter $\left(H_{*}\right)$ or temperature $\left(T_{*}\right)$ when the phase transitions completes, its inverse duration $(\beta)$, strength $(\alpha)$ at $T_{*}$ and the bubble wall velocity $\left(v_{w}\right)$. The exact definitions and derivation of these quantities are detailed in e.g. refs. [159, 176, 194], and in particular [195-200] for the bubble equations of motion and $v_{w}$. Also non-perturbative methods for nucleation have been developed [201, 202] as an alternative to perturbation theory.

Step (D): Numerical, large scale lattice simulations of relativistic hydrodynamics; cf. refs. [203208]. The parameters that describe the phase transition dynamics, $\left(T_{*}, \alpha, \beta / H_{*}, v_{w}\right)$, are input to simulations of colliding bubbles, cosmic fluid and sound waves after the 
phase transition completes. These determine the GW power spectrum. In practice, the approximate, analytical power spectrum has been solved from such simulations in terms of a generic set of input parameters. For an application of this, ref. [176] has devised the online tool PTPlot.

Step (E): A detectable GW background signature depends on the architecture of the detector in addition to the predicted stochastic GW power spectrum. The determination of the signal-to-noise ratio for a predicted signal at LISA is specified in ref. [176].

Step (F): A necessary condition for the EW baryogenesis [27] are first order phase transitions occurring via bubble nucleation. For reviews cf. [28, 29, 209]. The generation of a baryon asymmetry could be realised when new BSM sources of C and CP violation are invoked and baryon number violating sphaleron transitions are sufficiently suppressed in the broken phase. The latter can be associated with sufficiently strong transitions.

Next, we detail step (B) starting by a brief summary of the technique of hightemperature dimensional reduction.

\subsection{Dimensional reduction for a high-temperature 3d effective theory}

High-temperature dimensional reduction encodes the IR physics of the high-temperature plasma in an effective three-dimensional theory to describe long wavelength phenomena. In the context of electroweak theories, classic references are [111-113] but we also refer [210-212].

The equilibrium thermodynamics of a thermal field theory is described by an evolution in imaginary time $(\tau)$. Therein, bosonic (fermionic) fields satisfy (anti-)periodic boundary conditions with period $\tau=1 / T$ and can be decomposed into bosonic and fermionic Matsubara [213] modes

$$
\phi(\tau, \mathbf{k})=T \sum_{n=-\infty}^{\infty} \phi_{n}(\mathbf{k}) e^{i \omega_{n} \tau}, \quad \omega_{n}=\left\{\begin{array}{ll}
\omega_{n}^{\mathrm{B}}=2 n \pi T & \text { (bosons) } \\
\omega_{n}^{\mathrm{F}}=(2 n+1) \pi T & \text { (fermions) }
\end{array}, \quad n \in \mathbb{Z},\right.
$$

where $\mathbf{k}$ is a three-dimensional (3d) momentum. In other words the resulting theory is a $3 \mathrm{~d}$ one with an infinite tower of modes each carrying a mass $\omega_{n}^{2}$ corresponding to the Matsubara frequency of mode $n$.

This system can be studied in an effective theory formulation. In that EFT the central degree of freedom is the static bosonic $3 \mathrm{~d}$ zero mode $\left(\omega_{n=0}^{\mathrm{B}}\right)$ of the original four-dimensional (4d) field. The remaining non-zero modes of scale $\sim \pi T$ can been integrated out. This is the dimensional reduction step which is based on the high-temperature scale hierarchy

$$
\pi T \gg g T \gg g^{2} T / \pi
$$

In the scale hierarchy we introduced a power counting parameter $g$ defining the hard $(\pi T)$, soft $(g T)$, and ultrasoft $\left(g^{2} T / \pi\right)$ scales. While the scaling of the hard scale is a direct consequence of the Matsubara decomposition, the soft and ultrasoft scale are pertinent to collective plasma effects. Based on this hierarchy one can invoke a high-temperature 
expansion $m_{\psi} / T \ll 1$ for generic scalar fields $\psi$, whereas gauge bosons and fermions are massless in the unbroken phase.

In hindsight of the ensuing studies of a real scalar field, we establish the formal scaling $\lambda \sim g^{2}$ for the scalar quartic coupling $\lambda$ which is based on their appearance at one-loop. In gauge field theories this power counting parameter is often set to be the gauge coupling. For a scalar field we assume the original mass squared parameter $\left(\mu^{2}\right)$ to behave as $\mu^{2} \sim$ $\lambda T^{2} \sim(g T)^{2}$ which implies that the mass of the $3 \mathrm{~d}$ soft mode $\left(\mu_{3}^{2}\right)$ is thermally corrected by $\mu_{3}^{2} \simeq \mu^{2}+(g T)^{2}$ at leading order.

Phase transition physics can often be studied at the ultrasoft scale by a simplified $3 \mathrm{~d}$ EFT, where the soft scale has been integrated out. In fact, the transition point resides near a vanishing $\mu_{3}^{2}$ where thermal loop corrections cancel the tree-level part. At this point the $3 \mathrm{~d}$ mass scale is formally of the next natural order which is the ultrasoft one $\mu_{3}^{2} \sim\left(g^{2} T\right)^{2}$ where soft modes are screened. The corresponding soft degrees of freedom are the temporal (adjoint) scalar fields which are remnants of the zero components of gauge fields and induced by the broken Lorentz symmetry from the heat bath. They remain soft in the vicinity of the transition point. For this second step of dimensional reduction, see ref. [111].

The EFT is constructed by determining the operator coefficients of the effective Lagrangian. In practice, these parameters follow from matching correlation functions of both the fundamental $4 \mathrm{~d}$ theory and effective $3 \mathrm{~d}$ theory. The generic rules of this procedure were established in refs. [111-113] and applied recently [159, 160].

This construction of the 3d EFT by dimensional reduction is completely infrared-safe. In the matching of correlators, the IR and $3 \mathrm{~d}$ contributions cancel each other and only the hard scale (non-zero Matsubara modes) contributes. The corresponding sum-integrals over non-zero modes are IR-regulated by non-vanishing Matsubara frequencies at high temperature. Hence, the dimensional reduction defers the IR problem of high-temperature bosonic perturbation theory to the $3 \mathrm{~d}$ EFT.

At next-to-leading order (NLO) dimensional reduction, couplings are matched at oneloop and masses at two-loop level. This ensures a $\mathcal{O}\left(g^{4}\right)$ accuracy in the established power counting. To fully match this accuracy, the running parameters have to be related to physical observables at one-loop order in zero temperature perturbation theory. In $3 \mathrm{~d}$ perturbation theory the effective potential is computed at two-loop order. Notably, the frequently used $4 \mathrm{~d}$ daisy-resummed thermal effective potential at one-loop includes some — but crucially not all - $\mathcal{O}\left(g^{4}\right)$ contributions [104].

Instead of detailing the generic procedure in later sections, we choose an alternative approach. In an explicit hands-on demonstration, we dimensionally reduce a scalar field theory in section 3 , and generalise it to the XSM in section 4 and appendix A.

\subsection{Approaches to thermodynamics of thermal phase transitions}

Let us assess the main approaches to access the thermodynamics of the thermal phase transitions in electroweak theories. See also similar summaries in section 2 of ref. [137], section 2.2 of ref. [142] and section 1 of ref. [160]. The following approaches are illustrated in figure 2 : 


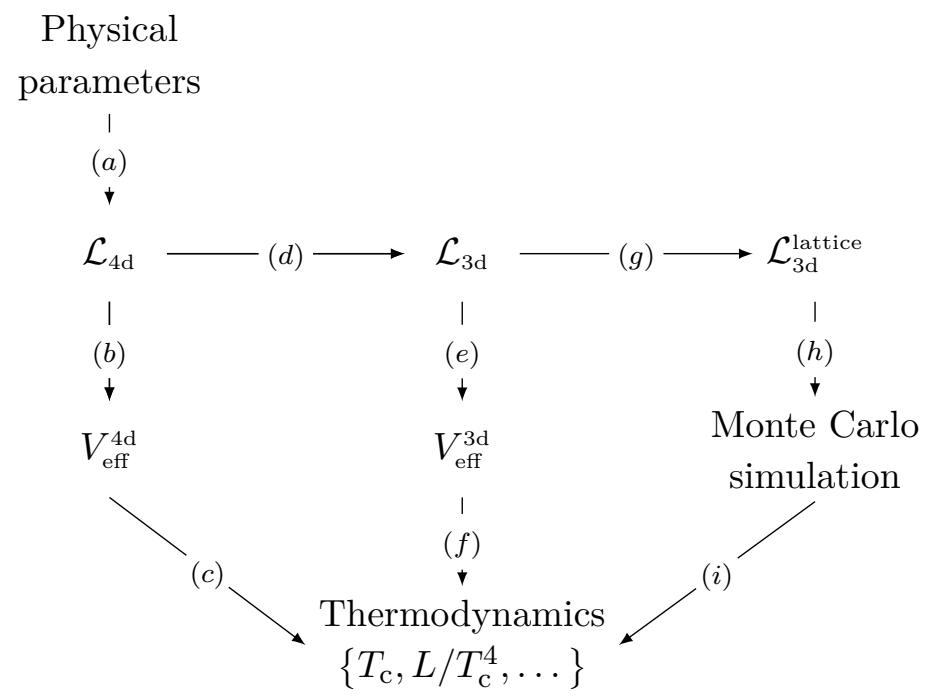

Figure 2. Three different approaches towards the thermodynamics of the electroweak phase transition. We focus on the purely perturbative $3 \mathrm{~d}$ approach with steps $(d)$ and $(e)$ for a real scalar theory and the Standard model supplemented by a real scalar singlet.

- “4d approach" $(a) \rightarrow(b) \rightarrow(c):$

Perturbative effective potential with daisy resummation.

- "Perturbative 3d approach" $(a) \rightarrow(d) \rightarrow(e) \rightarrow(f)$ :

Perturbative effective potential in 3d EFT.

- "Non-perturbative 3d approach" $(a) \rightarrow(d) \rightarrow(g) \rightarrow(h) \rightarrow(i)$ :

Non-perturbative lattice simulation of $3 \mathrm{~d}$ EFT. Robust approach combining perturbative dimensional reduction and non-perturbative (Monte Carlo) methods.

The individual steps encompass:

(a) Relating physical parameters (such as pole masses) and Lagrangian (running) parameters at zero temperature. Often the " $4 \mathrm{~d}$ approach" uses only tree-level relations (e.g. refs. [65, 214]), but in order to match the accuracy of dimensional reduction at NLO $\mathcal{O}\left(g^{4}\right)$, one-loop vacuum renormalisation is required [111, 158, 167].

(b) Perturbative computation of the thermal effective potential [161]. Frequently performed at one-loop, with leading order daisy resummation [104, 163]. Two-loop computations are discussed for e.g. in refs. [164, 165, 167, 168]. This computation suffers from the IR problem the most, and additionally can contain a dramatic artificial RG scale dependence if two-loop thermal masses are unaccounted [173].

(c) Computation of thermodynamics. At the critical temperature the minima of the effective potential are degenerate and thermodynamic quantities are obtained by differentiation with respect to temperature, viz. latent heat. Model-independent tools to locate degenerate minima have been implemented numerically in software including 
CosmoTransitions [215], BSMPT [214], and PhaseTracer [216]. It is worth noting that location of minima of the effective potential are not gauge invariant. Thus, this computation frequently introduces unphysical estimates for thermodynamics as discussed in refs. [159, 168, 217] and also ref. [218] (in 3d EFT context).

(d) Dimensional reduction to a 3d EFT. See refs. [111-113] and also recent refs. [159, 160]. It is perturbative and IR-safe, since only the hard scale is integrated out, and systematically implements all required resummations. Furthermore, dimensional reduction at NLO is analytically independent of the $4 \mathrm{~d}$ renormalisation scale up to that order, see refs. [111, 173]. This decreases the theoretical uncertainty in perturbation theory. A concrete computation is displayed in sections 3, 4 and appendix A.

(e) Computation of $3 \mathrm{~d}$ effective potential, see refs. [169, 170, 218]. The computation in the $3 \mathrm{~d}$ EFT simplifies significantly compared to $4 \mathrm{~d}$ because sum-integrals are replaced by vacuum integrals in $d=3-2 \epsilon$ spatial dimensions. Hence, it straightforwardly extends to two-loop order, cf. section 3.4. For recent applications, see refs. [154, 159]. Even the three-loop effective potential has been computed for a pure scalar theory [219] and applied recently [160].

(f) Computation of thermodynamics from 3d effective potential. Again a pathological gauge-dependent analysis can be based on degenerate minima at the transition point. However, also a gauge invariant treatment is possible, in terms of gauge invariant condensates $[105,159]$ or the pressure in $\hbar$-expansion. However, IR divergences arise at two-loop order for a radiatively generated transition [106], compromising the analysis [218]. On the other hand, these IR singularities are avoided in presence of a barrier at tree-level, and a manifestly gauge invariant treatment for the thermodynamics can be obtained in perturbation theory; see ref. [159].

(g) Lattice-continuum relations; see refs. [105, 106, 160, 220, 221]. The Lagrangian parameters of the lattice discretisation need to be related to those of the continuum theory. Thus, the results of Monte Carlo simulations can be associated with the $3 \mathrm{~d}$ continuum theory and via dimensional reduction to temperature and physical parameters. This can be done by computing and equating effective potentials in both discretisations, to two-loop order. In super-renormalisable theories without higher dimensional operators, all divergences arise at finite loop order and hence relations between continuum and lattice are exact. However, this aggravates in the presence of higher dimensional operators as the $3 \mathrm{~d}$ theory retains renormalisability but loses super-renormalisability. It remains a future challenge to overcome this technical issue. Note that in lattice gauge theories there is no need to fix the gauge, and the treatment is automatically gauge invariant by construction [222].

(h) Monte Carlo lattice simulations of spatial 3d EFT on finite volume and lattice spacing. ${ }^{3}$ Arbitrary field configurations are evolved - usually by a colourful cocktail of

\footnotetext{
${ }^{3}$ See refs. [21, 24, 105, 106, 114, 115, 124 $\left.126,138,139,142,144,146,154,158,160\right]$.
} 
update algorithms - to form a Markov chain converging to a Boltzmann probability distribution. Thereof, physical quantities can be measured such as scalar and gauge condensates, and correlation lengths. Many autocorrelation times are measured to ensure that statistical errors remain small. At the transition point, the system is equally likely to occur in any of the phases. Multi-canonical methods in first order transitions ensure that the system can efficiently sample all phases while not getting stuck in one.

(i) Extrapolate simulations of finite volume and fixed lattice spacing to the continuum. This corresponds to infinite volume and vanishing lattice spacing, thermodynamic and continuum limits, respectively. In practice, several lattice spacings are needed, each with several different volumes. This rapidly becomes computationally expensive and even a single parameter space point requires a large number of individual simulations. Furthermore, oftentimes manual effort is required to fit a proper continuum extrapolation to the data instead of an elephant.

This article focuses specifically on steps $(d)$ and $(e)$ which are detailed for a real scalar theory in section 3 and a real singlet scalar coupled to the SM in section 4 and appendix A. The full non-perturbative path of the real scalar theory is presented in ref. [160], where the corresponding results are compared with three-loop $3 \mathrm{~d}$ perturbation theory.

Finally, let us summarise the different approaches and describe some of their merits.

\subsubsection{4d approach}

The $4 \mathrm{~d}$ approach is the accustomed "bread and butter" approach with the advantage of its conceptual simplicity. At one-loop order, a closed form expression for the effective potential is available in terms of mass squared eigenvalues and one-loop thermal mass corrections, which straightforwardly automates to different models. In addition, numerical tools for thermodynamics (e.g. minimisation of potential) have been developed [214-216, 223] and can scan large regions parameter space of BSM models.

However, the $4 \mathrm{~d}$ approach suffers from the IR problem of perturbation theory [102] and is often plagued with large inaccuracies and theoretical uncertainties $[21,105,106,158$, 159]. In particular, weak transitions are poorly described by perturbation theory and are sometimes even qualitatively mistaken. Especially, crossover transitions are not predicted at all and it is not expected to determine the critical temperature accurately since it is highly IR-sensitive. Conversely, large couplings are often required for strong transition and can compromise the perturbative expansion, even at zero temperature [158]. Consistent $(\hbar-$ )expansions leading to gauge invariant results are oftentimes unavailable, since they require the knowledge of higher order contributions. Furthermore, a truncation of the computation already at one-loop order omits important thermal mass contributions at two-loop order. In turn, this causes a large leftover renormalisation group (RG) scale dependence, see ref. [173].

\subsubsection{Perturbative 3d approach}

Also this method still suffers from the IR problem of perturbation theory. We emphasise that for the perturbative effective potential itself, there is no real quantitative difference between $4 \mathrm{~d}$ and $3 \mathrm{~d}$ approaches, provided that in both cases the computation is performed 
to the same order in both coupling expansion and high- $T$ expansion. However, dimensional reduction systematically accesses higher order resummations and it is customary to include a consistent $\mathcal{O}\left(g^{4}\right)$ accuracy by a two-loop level computation, which yields a reduced RG scale dependence. A gauge dependence of the analysis can still be a theoretical blemish, but a gauge invariant treatment is possible by employing a $\hbar$-expansion and computing gauge invariant condensates $[105,159]$. Although radiatively induced transitions suffer from IR divergences at $\mathcal{O}\left(\hbar^{2}\right)[218]$.

As a downside, the perturbative $3 \mathrm{~d}$ approach is harder to automate and streamline compared to $4 \mathrm{~d}$ approach due to additional steps. Although the computation of the $3 \mathrm{~d}$ effective potential of a 3d EFT can be related to known topologies arising at two-loop order, the automation of dimensional reduction and the construction of the 3d EFT are still not common standard. For developments, see [159, 171, 224, 225]. In the future, automated dimensional reduction for multiple BSM theories could permit a perturbative $3 \mathrm{~d}$ approach to be implemented to software that currently relies on the $4 \mathrm{~d}$ approach.

\subsubsection{Non-perturbative 3d approach}

This method solves the IR problem, by treating perturbative (hard and soft) modes perturbatively while non-perturbative ultrasoft modes are analysed by lattice simulations. Furthermore, lattice simulations provide manifestly gauge invariant results. While this approach is very technical and computationally slow and demanding, it is still straightforward compared to direct $4 \mathrm{~d}$ simulations (see ref. [175] and references therein). Recent attempts $[154,158,160]$ simulate phase transitions in a limited number of BSM setups and benchmark points. The hope is to expose general trends regarding accuracy and reliability of simpler tools in perturbation theory. However, model-independent or conclusive results are unavailable so far and similar investigations are actively continued in the future. Finally, we highlight that the 3d EFT approach is also an applicable and attractive framework for non-equilibrium physics of phase transition, such as bubble nucleation and sphaleron rate; see refs. [201, 202, 226].

\section{Dimensional reduction with a real scalar: a tutorial}

The following tutorial constructs the dimensionally reduced 3d EFT of a single real scalar field. ${ }^{4}$ The machinery presented builds upon classic literature [111-113, 169] and generalises straightforwardly to more complicated BSM theories with non-minimal Higgs sector. To guide upcoming generalisations of complicated models, we detail step-by-step derivations that can be used for future crosschecks. Furthermore, based on advances of automation in thermal field theories [159, 171, 224, 225], we implemented in-house software in FORM [227] and applied qgraph [228] for diagram generation. Integration-by-parts reductions (IBP)

\footnotetext{
${ }^{4} \mathrm{An}$ independent computation [160] treats masses and tadpole as interactions in strict perturbation theory and in the unbroken phase. The dictionary $\left\{\mu_{1} \leftrightarrow \sigma, \mu_{\sigma}^{2} \leftrightarrow m^{2}, \mu_{3} \leftrightarrow \frac{g}{2}, \lambda_{\sigma} \leftrightarrow \frac{\lambda}{6}\right\}$ gives a direct comparison, wherein $g$ denotes the cubic coupling of the real scalar not to be confused with a formal power counting parameter or the $\mathrm{SU}(2)$ gauge coupling.
} 
follow a standard Laporta algorithm [229] adapted for thermal integrals [224]. This algorithmic perturbative treatment fully automates the computation of correlation functions within the unbroken phase and their matching. This software can tame the ever increasing complexity of computations in future models with multiple interacting new BSM fields.

The real singlet scalar model demonstrates all details of dimensional reduction. Without coupling it to the SM with the Higgs doublet, gauge fields, and fermions, this model poses an ideal starting point. The following computations employ explicit resummation to cancel delicate soft/hard mixing contributions at two-loop order. Practically, these IR contributions are trivially dropped [160] in strict perturbation theory. As an instructive crosscheck, we perform the computation both in the broken phase using the effective potential, the generator of correlators, as well as the unbroken phase computing correlators directly.

Section 4 focusses on full xSM - where a real singlet scalar is coupled to the SM Higgs - and presents the definition of the EFT including results. Details of this full computation are relegated to appendix $\mathrm{A}$ and results of appearing (sum-)integrals are collected in appendix B. For the derivation of such integrals, we refer refs. [224, 230, 231]. Our notation follows ref. [151] in which dimensional reduction for the xSM was initially discussed. This reference deferred the case of a light ("soft") singlet which remains dynamical in the 3d EFT to our computation.

\subsection{Model and parameter matching}

Consider the theory of a single real scalar field $\sigma$, given by bare $4 \mathrm{~d}$ Lagrangian (with Euclidean metric) in the imaginary time formalism

$$
\mathcal{L}=\frac{1}{2}\left(\partial_{\mu} \sigma_{(b)}\right)^{2}+\frac{1}{2} \mu_{\sigma(b)}^{2} \sigma_{(b)}^{2}+\mu_{1(b)} \sigma_{(b)}+\frac{1}{3} \mu_{3(b)} \sigma_{(b)}^{3}+\frac{1}{4} \lambda_{\sigma(b)} \sigma_{(b)}^{4} .
$$

Definitions of bare quantities in terms of their renormalised versions and counterterms in renormalised perturbation theory are found in section 2.1 of ref. [151]. Note that we choose a general renormalisable theory with linear and cubic terms without $Z_{2}$-symmetry $\sigma \rightarrow-\sigma$. Conveniently, in the simple case of a real scalar (without gauge fields) the $3 \mathrm{~d}$ EFT and $4 \mathrm{~d}$ parent theory bear the same form. With the exception that after dimensional reduction the couplings and field live in a spatial $3 \mathrm{~d}$ theory. We organise our perturbative expansion by establishing the following formal power counting

$$
\mu_{1} \sim g T^{3}, \quad \mu_{\sigma}^{2} \sim g^{2} T^{2}, \quad \mu_{3} \sim g T, \quad \lambda_{\sigma} \sim g^{2},
$$

where $g$ is a formal power counting parameter that corresponds to the weak coupling at zero temperature. Once this theory couples to the SM in section 4, the formal power counting parameter $g$ is identified as the $\mathrm{SU}(2)$ gauge coupling. Within the power counting (3.2), we aim for a dimensional reduction at NLO with $\mathcal{O}\left(g^{4}\right)$ accuracy. At loop-level, this requires one-loop accuracy for cubic and quartic couplings, and two-loop order for tadpole and mass parameter.

We emphasise that the above formal choice for the scaling of the cubic coupling leads to a peculiarity illustrated along the tree-level quartic interactions induced by the cubic 


$$
\begin{aligned}
& \left(\sigma^{2}\right)_{3 \mathrm{~d}}=\frac{1}{T}\left(\sigma^{2}\right)_{4 \mathrm{~d}}\left(1+\hat{\Pi}_{\sigma^{2}}^{\prime}\right) \\
& =\frac{1}{T}\left(\sigma^{2}\right)_{4 \mathrm{~d}}\left(1+\frac{\mathrm{d}}{\mathrm{d} K^{2}}=(1)=\right.
\end{aligned}
$$

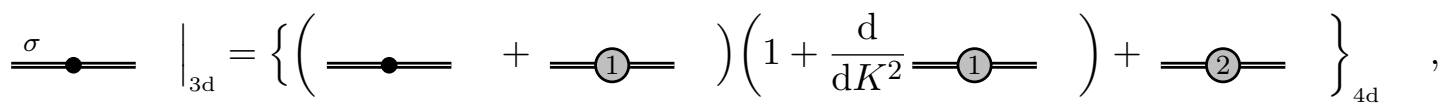

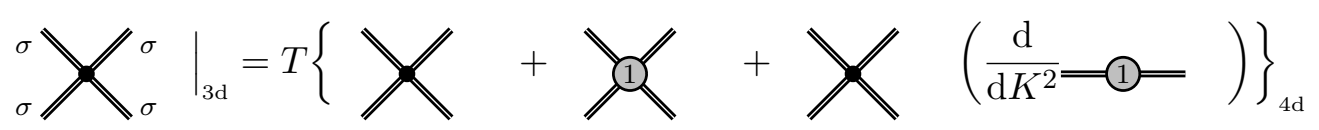

Figure 3. Illustration of a $\mathcal{O}\left(g^{4}\right)$ NLO matching of correlators between $3 \mathrm{~d}$ and $4 \mathrm{~d}$ theories with $Z_{2}$-symmetry. A full non- $Z_{2}$-symmetric case is analogous. Blobs present the sum of hard contributions to one- and two-loop diagrams in perturbation theory, and the differentiation (prime) acts upon the external soft momentum $K=(0, \mathbf{k})$. The diagrams were drawn with Axodraw [233].

coupling. Its contribution

$$
\ \simeq \frac{\mu_{3}^{2}}{\mu_{\sigma}^{2}} \sim \mathcal{O}(1) \gg \lambda_{\sigma}
$$

parametrically dominates over the corresponding quartic coupling, and could even compromise perturbativity at zero temperature. In practice for dimensional reduction, this causes no complications as the above interaction is 1-particle reducible. Hence, it is absent in Green's functions that are matched during dimensional reduction for the ultrasoft (light) field in 3d EFT. By enforcing a different scaling, namely $\mu_{3} \sim g^{2} T$, the contribution (3.3) formally scales as the corresponding quartic coupling. However, this suppresses almost all contributions of $\mu_{3}$ in the matching relations at $\mathcal{O}\left(g^{4}\right)$.

Hence, our strategy is the following: for generality we indeed install a scaling of $\mu_{3} \sim$ $g T$ and include all contributions of cubic couplings in our matching relations. These contributions can always be trivially dropped if an extra suppression is assumed. This choice allows us to more widely illustrate different aspects of the dimensional reduction procedure, such as effects from field normalisation. Indeed, a non- $Z_{2}$-symmetric theory can demonstrate the high-temperature screening of the fields by the hard scale via ring topology diagrams such as in eq. (3.7). These are absent in a $Z_{2}$-symmetric case. The motivation to include these contributions is the presence of similar diagrams in theories with gauge fields and fermions, even if the scalar sector is $Z_{2}$-symmetric.

Figure 3 illustrates the NLO matching of the parameters. For references with explicit matching examples, see refs. [111-113, 151, 159, 160]. Loop corrections from the $3 \mathrm{~d}$ side and soft contributions match exactly. Hence both drop out trivially in the matching, leaving only hard contributions. At two-loop order mixed soft/hard terms are cancelled by counterterm-like resummation interactions at one-loop [111, 157]. We demonstrate this in section 3.2.3. 
For example, the matching of correlation functions for the quartic self-interaction in both theories yields

$$
\underbrace{T\left(-6 \lambda_{\sigma, 3}-\left\langle\sigma^{4}\right\rangle_{\text {1loop }}^{\text {3d }}\right)}_{\text {3d }}=\underbrace{-6 \lambda_{\sigma}-\left\langle\sigma^{4}\right\rangle_{1 \text { loop }}^{\text {soft }}-\left\langle\sigma^{4}\right\rangle_{\text {1loop }}^{\text {hard }}}_{4 \mathrm{~d}} .
$$

Note that here the correlators equal minus the sum of the tree-level vertex and Feynman diagrams at higher orders. For a consistent matching, the resummation of parameters (see details in eq. (3.16)) for zero modes allows to identify $3 \mathrm{~d}$ loop corrections with soft terms in the $4 \mathrm{~d}$ computation, and these two (IR) terms cancel. Furthermore, resummation ensures a cancellation of all mixed soft/hard mode contributions in one- and two-point correlation functions at two-loop order. For details, see eqs. (3.27) and (3.42)-(3.46). The effective vertices in both theories read

$$
T \lambda_{\sigma, 3} \sigma_{3 \mathrm{~d}}^{4}=\left(\lambda_{\sigma}+\frac{1}{6}\left\langle\sigma^{4}\right\rangle_{1 \text { loop }}^{\text {hard }}\right) \sigma_{4 \mathrm{~d}}^{4}
$$

and relating $3 \mathrm{~d}$ and $4 \mathrm{~d}$ fields by the first line of figure 3 leads to a $\mathcal{O}\left(g^{4}\right)$ result

$$
T \lambda_{\sigma, 3}=T\left(\lambda_{\sigma}+\frac{1}{6}\left\langle\sigma^{4}\right\rangle_{1 \text { loop }}^{\text {hard }}-2 \lambda_{\sigma} \Pi_{2}^{\prime}\right) .
$$

Matching relations of other effective parameters are obtained analogously.

The $4 \mathrm{~d}$ and $3 \mathrm{~d}$ fields at one-loop level are related by a computation of the ring topology diagram at non-zero external static momentum $K=(0, \mathbf{k})$ with soft $|\mathbf{k}|=k \sim g T$. Denoting sum-integrals according to appendix B, a series expansion to quadratic order yields

$$
\begin{aligned}
\Pi_{2}^{\prime} & \equiv \frac{\mathrm{d}}{\mathrm{d} k^{2}} \Pi_{2}(k)=\frac{\mathrm{d}}{\mathrm{d} k^{2}}= \\
& =\frac{\mathrm{d}}{\mathrm{d} k^{2}}\left(-2 \mu_{3}^{2} \xi_{P}^{\prime} \frac{1}{P^{2}(P+K)^{2}}\right) \\
& =\frac{\mathrm{d}}{\mathrm{d} k^{2}}\left(-2 \mu_{3}^{2} \oint_{P}^{\prime} \frac{1}{P^{2}}\left(\frac{1}{P^{2}}-2 \frac{\mathbf{k} \cdot \mathbf{p}}{P^{4}}+4 \frac{(\mathbf{k} \cdot \mathbf{p})^{2}}{P^{6}}-k^{2} \frac{1}{P^{4}}+\mathcal{O}\left(k^{3}\right)\right)\right) \\
& =2 \mu_{3}^{2} \oint_{P}^{\prime}\left(\frac{d-4}{d} \frac{1}{P^{6}}+\frac{4}{d} \frac{P_{0}^{2}}{P^{8}}\right) \\
& =\frac{2}{3} \mu_{3}^{2} \oiint_{P}^{\prime} \frac{1}{P^{6}}=2 \mu_{3}^{2} \frac{1}{(4 \pi)^{4}} \frac{2}{3} \frac{\zeta_{3}}{T^{2}},
\end{aligned}
$$

where we interchangeably denote $\Pi_{n}=\left\langle\sigma^{n}\right\rangle$ as correlation functions and utilise the intact $d$-dimensional rotational symmetry $(\mathbf{k} \cdot \mathbf{p})^{2} \rightarrow \frac{1}{d} k^{2} p^{2}$, trivial manipulation $p^{2}=P^{2}-P_{0}^{2}$ and integration-by-parts relations $\mathscr{L}_{P} \frac{\left(P_{0}^{2}\right)^{\beta+1}}{\left[P^{2}\right]^{\alpha+1}}=\left(1-\frac{d}{2 \alpha}\right) \mathscr{\mathscr { S }}_{P} \frac{\left(P_{0}^{2}\right)^{\beta}}{\left[P^{2}\right]^{\alpha}}$.

The effective potential generates all correlation functions at zero external momenta. In cases where an explicit momentum dependence exceeds the accuracy of the computation, accessing correlators simplifies greatly by starting from the effective potential. Shifting the scalar field $\sigma \rightarrow \sigma+s$ with a real background field $s$, the effective potential reads

$$
V_{\text {eff }}=\sum_{n=1}^{\infty} \frac{\left\langle\sigma^{n}\right\rangle}{n !} s^{n} \equiv V_{1} s+\frac{1}{2} V_{2} s^{2}+\frac{1}{3} V_{3} s^{3}+\frac{1}{4} V_{4} s^{4}+\ldots
$$


where the ellipsis truncates potential higher dimensional correlators that lead to $\mathcal{O}\left(g^{5}\right)$ marginal operators in the EFT.

The complete matching relations that define the dimensionally reduced 3d EFT, encode the thermodynamics of the original $4 \mathrm{~d}$ theory of eq. (3.1) at $\mathcal{O}\left(g^{4}\right)$. They read

$$
\begin{aligned}
& \mu_{1,3}=T^{-\frac{1}{2}}\left(V_{1}^{2 \text { loop }}-\frac{1}{2} V_{1}^{1 \text { loop }} \Pi_{2}^{\prime}\right), \\
& \mu_{\sigma, 3}^{2}=V_{2}^{\text {2loop }}-V_{2}^{1 \text { loop }} \Pi_{2}^{\prime}, \\
& \mu_{3,3}=T^{\frac{1}{2}}\left(V_{3}^{1 \text { loop }}-\frac{3}{2} \mu_{3} \Pi_{2}^{\prime}\right), \\
& \lambda_{\sigma, 3}=T\left(V_{4}^{1 \text { loop }}-2 \lambda_{\sigma} \Pi_{2}^{\prime}\right),
\end{aligned}
$$

where we indicated the required loop order for a $\mathcal{O}\left(g^{4}\right)$ accuracy. The coefficients $V$ merely contain hard contributions and correspond to correlators via eq. (3.8).

\subsection{Computation of correlators}

The computation of $n$-point correlation functions is expounded in two different ways. In the following, we discuss their merits. In the broken phase, the computation uses the mass eigenstate basis and we employ the effective potential which is the generator of the correlation functions. Therein, the scalar field is shifted by a classical background field. In the unbroken phase, in the gauge eigenstate basis, we compute all correlators directly diagram-by-diagram.

These two approaches give rise to an equivalent result. Technically, for a real scalar theory the broken and unbroken phase computations vary marginally. However, subtleties of these two approaches become more prominent for gauge field theories with (multiple) scalars in different representations of the underlying gauge symmetry group.

\subsubsection{Broken phase: correlators from the two-loop effective potential}

Within the broken phase computation, the Feynman rules for vertices read

$$
\begin{aligned}
& V_{\sigma^{3}}=-3 !\left(\frac{\mu_{3}}{3}+s \lambda_{\sigma}\right), \\
& V_{\sigma^{4}}=-4 !\left(\frac{\lambda_{\sigma}}{4}\right) .
\end{aligned}
$$

Denoting the four-momentum by $P=\left(P_{0}, \mathbf{p}\right)$, where $P_{0}=2 \pi n T$ for each bosonic Matsubara mode, the free scalar propagator is

$$
\langle\sigma(P) \sigma(Q)\rangle=\frac{\delta(P+Q)}{P^{2}+m^{2}},
$$

and employs the notation $\delta(K) \equiv T^{-1} \delta_{K_{0}, 0}(2 \pi)^{d} \delta^{(d)}(\mathbf{k})$ where $\delta_{P_{0}} \equiv \delta_{P_{0}, 0}$ denotes the Kronecker delta for vanishing zero mode. The broken phase mass parameter $m^{2}=\mu_{\sigma}^{2}+$ 
$2 s \mu_{3}+3 \lambda_{\sigma} s^{2}$ appearing in the propagator corresponds to the squared mass eigenvalue of field $s$. The resummation of the zero mode $\sigma_{0}$ writes $^{5}$

$$
\begin{aligned}
\mathcal{L}= & \left(\mathcal{L}_{\text {free }}+\frac{1}{2} \Pi_{2}^{1 \text { loop }} \sigma_{0}^{2}+\frac{1}{3} \Pi_{3}^{1 \text { loop }} \sigma_{0}^{3}+\frac{1}{4} \Pi_{4}^{1 \text { loop }} \sigma_{0}^{4}\right) \\
& +\left(\mathcal{L}_{\text {int }}-\frac{1}{2} \Pi_{2}^{1 \text { loop }} \sigma_{0}^{2}-\frac{1}{3} \Pi_{3}^{1 \text { loop }} \sigma_{0}^{3}-\frac{1}{4} \Pi_{4}^{1 \text { loop }} \sigma_{0}^{4}\right)
\end{aligned}
$$

where $\Pi_{n}$ contain hard mode corrections. Terms with plus signs resum the zero mode mass and terms with minus sign act as interactions. In particular, we have a quadratic resummation interaction

$$
V_{\sigma_{0}^{2}}=\Pi_{s} \equiv \Pi_{2}+2 s \Pi_{3}+3 s^{2} \Pi_{4},
$$

for the zero modes. We also have a UV counterterm interaction for all modes

$$
V_{\sigma^{2}}=-\left(\delta m^{2}+P^{2} \delta Z_{\sigma}\right)
$$

where $\delta m^{2}=\delta \mu_{\sigma}^{2}+2 s \delta \mu_{3}+3 \delta \lambda_{\sigma} s^{2}$ and $\delta Z_{\sigma}=0$ at one-loop level. After resummation the propagator reads

$$
\langle\sigma(P) \sigma(Q)\rangle=\frac{\delta(P+Q)}{P^{2}+m^{2}+\delta_{P_{0}} \Pi_{s}} .
$$

Perturbation theory is organised order-by-order. Thus, thermal corrections $\Pi_{n}$ at one-loop are needed explicitly for resummation at two-loop level. Therefore, we already quote the result

$$
\begin{aligned}
& \Pi_{2}=T^{2} \frac{\lambda_{\sigma}}{4}-2 \mu_{3}^{2} \frac{L_{b}}{(4 \pi)^{2}}, \\
& \Pi_{3}=-9 \mu_{3} \lambda_{\sigma} \frac{L_{b}}{(4 \pi)^{2}}, \\
& \Pi_{4}=-9 \lambda_{\sigma}^{2} \frac{L_{b}}{(4 \pi)^{2}} .
\end{aligned}
$$

The effective potential including two-loop level reads

$$
V_{\text {eff }}^{4 \mathrm{~d}}=V_{\text {tree }}+V_{\mathrm{CT}}+V_{1 \text { loop }}+V_{\text {2loop }}
$$

and even though counterterm and resummation diagrams are one-loop topologies they contribute at equal order as two-loop topologies. Figure 4 illustrates the corresponding two-loop level diagrams. The separate terms in the potential yield

\footnotetext{
${ }^{5}$ This (order-by-order) resummation identifies IR contributions by relating soft $4 \mathrm{~d}$ loop contributions with 3 d ones. The soft/hard mixing terms cancel explicitly at two-loop order. For gauge fields this procedure becomes technically complicated (cf. ref. [157]) and ref. [111] indicates that such an explicit resummation is somewhat cosmetical. While IR contributions in the matching must be identified, their specific expressions are obsolete. Therefore, soft/hard mixing terms never appear in the matching of strict perturbation theory [160], following refs. [112, 113].
} 


\section{$\infty \ominus \odot \odot$}

Figure 4. Two-loop level diagrams for the effective potential in $4 \mathrm{~d}$ perturbation theory. The last two diagrams comprise renormalisation and resummation counterterms with quadratic interactions, respectively.

$$
\begin{aligned}
V_{\text {tree }}= & \frac{1}{2} \mu_{\sigma} s^{2}+\mu_{1} s+\frac{1}{3} \mu_{3} s^{3}+\frac{1}{4} \lambda_{\sigma} s^{4} \\
V_{\mathrm{CT}}= & \frac{1}{2} \delta \mu_{\sigma}^{2} s^{2}+\delta \mu_{1} s+\frac{1}{3} \delta \mu_{3} s^{3}+\frac{1}{4} \delta \lambda_{\sigma} s^{4} \\
V_{1 \text { loop }}= & J_{\text {soft }}\left(m_{3 \mathrm{~d}}\right)+J_{\text {hard }}(m) \\
V_{\text {2loop }}= & -\left(\frac{1}{8} V_{\sigma^{4}}(-1) \mathcal{D}_{S S}(m, m)+\frac{1}{12} V_{\sigma^{3}}^{2} \mathcal{D}_{S S S}(m, m, m)\right. \\
& \left.\quad+\frac{1}{2}(-1) \mathcal{D}_{S}(m)+\frac{1}{2} \Pi_{s} I_{1}^{3}\left(m_{3 \mathrm{~d}}\right)\right)
\end{aligned}
$$

where $m_{3 \mathrm{~d}}^{2}$ corresponds to the mass eigenvalue in the $3 \mathrm{~d}$ theory. Therein, all master integrals are defined in appendix B in the high- $T$ expansion and in dimensional regularisation utilising the $\overline{\mathrm{MS}}$-scheme. On the UV side, all $T^{2}$-independent $1 / \epsilon$ and $1 / \epsilon^{2}$ poles cancel in dimensional regularisation. On the IR-sensitive side, non-analytic, mixed soft/hard terms $\propto \sqrt{m_{3 \mathrm{~d}}^{2}}$ cancel due to resummation.

Expanding the effective potential in $\epsilon$ and the background field $s$ (cf. eq. (3.8)) results in

$$
\begin{aligned}
V_{1}= & \frac{1}{(4 \pi)^{2}} \frac{T^{2}}{\epsilon} \frac{1}{2} \lambda_{\sigma} \mu_{3}+\mu_{1}(\Lambda)+\left[\frac{1}{12} T^{2} \mu_{3}(\Lambda)-\frac{L_{b}}{(4 \pi)^{2}} \mu_{3} \mu_{\sigma}(\Lambda)\right]_{1 \text { loop }} \\
& +\left[\frac{L_{b}}{(4 \pi)^{2}} \frac{3}{4} T^{2} \lambda_{\sigma} \mu_{3}+\frac{1}{(4 \pi)^{4}} \mu_{3}^{3}\left(3+2 L_{b}+L_{b}^{2}\right)-\frac{1}{(4 \pi)^{2}} 2 \lambda_{\sigma} \mu_{3}\left(c+\ln \left(\frac{3 T}{\Lambda}\right)\right)\right]_{2 \text { loop }} \\
V_{2}= & \frac{1}{(4 \pi)^{2}} \frac{T^{2}}{\epsilon} \frac{3}{2} \lambda_{\sigma}^{2}+\mu_{\sigma}^{2}(\Lambda) \\
& +\left[\frac{1}{4} T^{2} \lambda_{\sigma}(\Lambda)-\frac{L_{b}}{(4 \pi)^{2}}\left(2 \mu_{3}^{2}(\Lambda)+3 \lambda_{\sigma} \mu_{\sigma}(\Lambda)\right)+\frac{\zeta_{3}}{(4 \pi)^{4} T^{2}} 8 \mu_{3}^{2} \mu_{\sigma}^{2}(\Lambda)\right]_{1 \mathrm{loop}} \\
& +\left[-T^{2} \frac{L_{b}}{(4 \pi)^{2}} \frac{9}{4} \lambda_{\sigma}^{2}+\frac{1}{(4 \pi)^{4}} \lambda_{\sigma} \mu_{3}^{2}\left(45+3 L_{b}\left(10+L_{b}\right)+2 \zeta_{3}\right)\right. \\
& \left.-\frac{\zeta_{3}}{(4 \pi)^{6} T^{2}} 8\left(3+2 L_{b}\right) \mu_{3}^{4}-\frac{1}{(4 \pi)^{2}} 6 \lambda_{\sigma}^{2}\left(c+\ln \left(\frac{3 T}{\Lambda}\right)\right)\right]_{2 \mathrm{loop}} \\
V_{3}= & \mu_{3}-\frac{L_{b}}{(4 \pi)^{2}} 9 \lambda_{\sigma} \mu_{3}+\frac{\zeta_{3}}{(4 \pi)^{4} T^{2}} 8 \mu_{3}^{3} \\
V_{4}= & \lambda_{\sigma}-\frac{L_{b}}{(4 \pi)^{2}} 9 \lambda_{\sigma}^{2}+\frac{\zeta_{3}}{(4 \pi)^{4} T^{2}} 48 \lambda_{\sigma} \mu_{3}^{2}-\frac{\zeta_{5}}{(4 \pi)^{6} T^{4}} 32 \mu_{3}^{4}
\end{aligned}
$$


employing abbreviations for thermal logarithms

$$
\begin{aligned}
c & =\frac{1}{2}\left(\ln \left(\frac{8 \pi}{9}\right)+\frac{\zeta_{2}^{\prime}}{\zeta_{2}}-2 \gamma_{\mathrm{E}}\right), \\
L_{b} & \equiv 2 \ln \left(\frac{\Lambda}{T}\right)-2\left(\ln (4 \pi)-\gamma_{\mathrm{E}}\right), \quad L_{f} \equiv L_{b}+4 \ln 2,
\end{aligned}
$$

in which $\Lambda$ is the $4 \mathrm{~d}$ renormalisation scale and $\gamma_{\mathrm{E}}$ the Euler-Mascheroni constant. The uncancelled $T^{2}$-dependent divergences correspond to the two-loop $3 \mathrm{~d}$ counterterms in eqs. (3.59) and (3.60).

One hallmark of the broken phase computation is its diagrammatic simplicity: the combinatorics of permuting external legs is intrinsic in the effective potential. As a drawback one has to evaluate massive sum-integrals at two-loop level to generate the dependence on the background field. Even though reaching $\mathcal{O}\left(g^{4}\right)$ the mass parameter $\mu_{\sigma}^{2}$ itself will not appear within two-loop pieces of the matching relations. This detail facilitates the unbroken phase computation in the next section. As another drawback, in models with multiple scalars, multiple background fields appear and it can be tedious to obtain an analytic series expansion for the effective potential in these background fields. This poses a complication, since an expansion in background fields, analogous to eq. (3.8), is needed to extract the correlators.

\subsubsection{Unbroken phase: correlators from the diagrammatic approach}

An alternative approach computes the correlation functions directly diagram-by-diagram (cf. footnote 4). The downside of this approach is its large number of diagrams with several permutations of external legs. Conversely, its extension to more complicated models is conceptually straightforward and even multiple gauge fields and scalars coupling to them can be handled algorithmically. This poses an advantage compared to the aforementioned complications in the broken phase where series expansions in (multiple) background fields were needed. In turn, at two-loop level one can set all propagators massless for the NLO dimensional reduction at $\mathcal{O}\left(g^{4}\right)$ in analogy to strict perturbation theory (cf. ref. [160]).

Within the unbroken phase computation, the Feynman rules for vertices read

$$
\begin{aligned}
& V_{\sigma^{3}}=-3 !\left(\frac{\mu_{3}+\delta \mu_{3}}{3}\right), \\
& V_{\sigma^{4}}=-4 !\left(\frac{\lambda_{\sigma}+\delta \lambda_{\sigma}}{4}\right), \\
& V_{\sigma^{2}}=-\left(\delta \mu_{\sigma}^{2}+P^{2} \delta Z_{\sigma}\right) .
\end{aligned}
$$

Note that the tadpole $\mu_{1}$ never contributes to 1PI diagrams required for the matching. The scalar propagator reads

$$
\langle\sigma(P) \sigma(Q)\rangle=\frac{\delta(P+Q)}{P^{2}+\mu_{\sigma}^{2}} .
$$

As mentioned, aiming for $\mathcal{O}\left(g^{4}\right)$ accuracy allows to treat propagators inside two-loop diagrams as massless. This provides the correct hard mode parts and non-analytic IR sensitive contributions vanish trivially in dimensional regularisation due to an absent mass scale. 
The 3-point and 4-point correlator consist of the following diagrams including their results in terms of master integrals (cf. appendix B)

$$
=-2 \delta \mu_{3},
$$

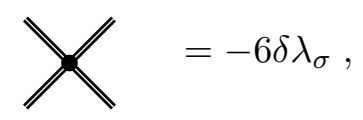

$$
\bigcirc=\frac{1}{2} \times 3 \times 36 \lambda_{\sigma}^{2} I_{2}^{4 b} \text {, }
$$$$
\simeq=1 \times 6 \times(-24) \mu_{3}^{2} \lambda_{\sigma} I_{3}^{4 b}
$$

$$
\bigcirc=1 \times 3 \times 16 \mu_{3}^{4} I_{4}^{4 b} \text {, }
$$

where we indicated symmetry factors and combinatorial factors related to permutations of external legs. The tadpole (1-point) correlator up to two-loop level yields

$$
\begin{aligned}
& \Longrightarrow \quad=-\delta \mu_{1} \text {, } \\
& =-2 \mu_{3}^{3} S_{4} \text {, } \\
& ==-\mu_{3}\left(I_{1}^{4 b}-\mu_{\sigma}^{2} I_{2}^{4 b}\right), \\
& \Longrightarrow=-\delta \mu_{3} I_{1}^{4 b}, \\
& =\bigcirc=3 \mu_{3} \lambda_{\sigma} I_{1}^{4 b} I_{2}^{4 b} \\
& =\mu_{3}\left(\delta Z_{\sigma} I_{1}^{4 b}+\delta \mu_{\sigma}^{2} I_{2}^{4 b}\right) \\
& \Longrightarrow=2 \mu_{3} \lambda_{\sigma} S_{3},
\end{aligned}
$$

Finally, the diagrammatic expressions of the self-energy (2-point correlator) up to two-loop level read

$$
=-\delta \mu_{\sigma}^{2},
$$

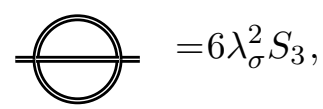$$
=\bigcirc=-6 \mu_{3}^{2} \lambda_{\sigma} I_{2}^{4 b} I_{2}^{4 b}
$$

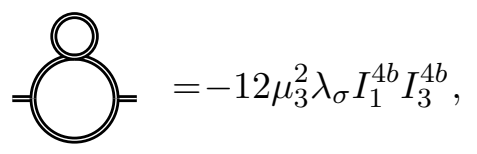

$$
\Longrightarrow=-24 \mu_{3}^{2} \lambda_{\sigma} S_{4}
$$




$$
\begin{gathered}
c=-6 \mu_{3}^{2} \lambda_{\sigma} S_{4}, \\
==8 \mu_{3}^{4} S_{6}, \\
=-3 \delta \lambda_{\sigma} I_{1}^{4 b},
\end{gathered}
$$$$
\Longrightarrow=4 \mu_{3} \delta \mu_{3} I_{1}^{4 b}
$$$$
\Longrightarrow=3 \lambda_{\sigma}\left(\delta Z_{\sigma} I_{1}^{4 b}+\delta \mu_{\sigma}^{2} I_{2}^{4 b}\right)
$$$$
==-4 \mu_{3}^{2}\left(\delta Z_{\sigma} I_{2}^{4 b}+\delta \mu_{\sigma}^{2} I_{3}^{4 b}\right)
$$

After summing individual diagrams for each correlator, we apply integrals of appendix B and recover the correlators of eqs. (3.28)-(3.31). Recall that the correlator itself is minus the sum of diagrams and within our convention $V_{n}=\left\langle\sigma^{n}\right\rangle /(n-1)$ ! given in eq. (3.8).

\subsubsection{Cancellation of mixed hard/soft terms}

As mentioned earlier, explicit resummation is obsolete since all propagators at two-loop diagrams are treated massless. To this end, we demonstrate how resummation unfolds as a cancellation of IR sensitive mixed hard/soft terms while keeping sum-integrals massive. For simplicity, we discuss the $Z_{2}$-symmetric case and employ the resummation

$$
\begin{aligned}
\mathcal{L}= & \left(\mathcal{L}_{\text {free }}+\frac{1}{2} \Pi_{2}^{1 \text { loop }} \sigma_{0}^{2}+\frac{1}{4} \Pi_{4}^{1 \text { loop }} \sigma_{0}^{4}\right) \\
& +\left(\mathcal{L}_{\text {int }}-\frac{1}{2} \Pi_{2}^{1 \text { loop }} \sigma_{0}^{2}-\frac{1}{4} \Pi_{4}^{1 \text { loop }} \sigma_{0}^{4}\right) .
\end{aligned}
$$

From the quadratic part we can read off the resummed propagator

$$
\langle\sigma(P) \sigma(Q)\rangle=\frac{\delta(P+Q)}{P^{2}+\mu_{\sigma}^{2}+\delta_{P_{0}} \Pi_{2}^{1 \text { loop }}}
$$

and the resummed vertex for the pure zero modes becomes $\lambda_{\sigma} \rightarrow \lambda_{\sigma}+\Pi_{4}$. Also resummation interaction terms are introduced for the zero mode

$$
\begin{aligned}
& V_{\sigma_{0}^{2}}=\Pi_{\sigma}, \\
& V_{\sigma_{0}^{4}}=6 \Pi_{\sigma} .
\end{aligned}
$$

At two-loop order, the two diagrams that contribute are (d.4) and (d.5). By expanding the latter, massive sunset integral in (d.4), at high- $T$

$$
\begin{aligned}
£_{P, Q} \frac{1}{\left[P^{2}+m^{2}\right]\left[Q^{2}+m^{2}\right]\left[(P+Q)^{2}+m^{2}\right]} & =T^{2} \int_{p, q} \frac{1}{\left[p^{2}+m^{2}\right]\left[q^{2}+m^{2}\right]\left[(p+q)^{2}+m^{2}\right]} \\
& +3 T \int_{p} \frac{1}{\left[p^{2}+\mu_{\sigma, 3}^{2}\right]} \oint_{Q}^{\prime} \frac{1}{Q^{4}}+\mathcal{O}\left(\mu_{\sigma}^{2}\right) \\
& \rightarrow 6 \lambda_{\sigma}^{2}(\underbrace{3 T I_{1}^{3}\left(\mu_{\sigma, 3}\right) I_{2}^{4 b}}_{(A)}),
\end{aligned}
$$




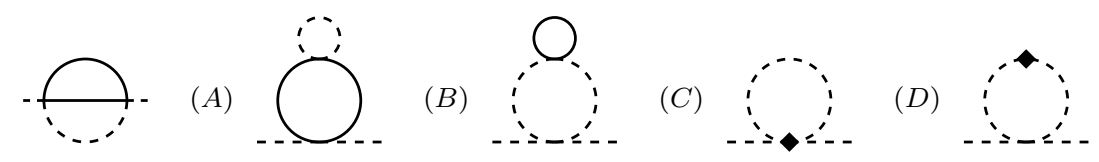

Figure 5. Resummation in the unbroken phase computation with $Z_{2}$ symmetry. Solid (dashed) lines denote scalar propagators with hard (soft) momenta. These IR-sensitive non-analytic mixed soft/hard contributions are compensated by resummation interaction diagrams (diamond). Alternatively, massless propagators in two-loop diagrams simplify the computation and retain the same result for the hard mode contribution relevant to matching.

one observes that masses are expanded in the pure hard terms but are kept in the mixed soft/hard terms. In fact, the resummed $3 \mathrm{~d}$ mass equals the one-loop dimensionally reduced mass parameter. The last line above reintroduced numerical factors and the scalar selfcoupling. Similarly, the bubble integral (d.4) yields

$$
\begin{aligned}
\sum_{P} \frac{1}{\left[P^{2}+\mu_{\sigma}^{2}\right]} \sum_{Q} & \frac{1}{\left[Q^{2}+\mu_{\sigma}^{2}\right]^{2}} \\
& =\left(T \int_{p} \frac{1}{\left[p^{2}+\mu_{\sigma, 3}^{2}\right]}+\sum_{P}^{\prime} \frac{1}{P^{2}}\right)\left(T \int_{q} \frac{1}{\left[q^{2}+\mu_{\sigma, 3}^{2}\right]^{2}}+\int_{Q}^{\prime} \frac{1}{Q^{4}}\right)+\mathcal{O}\left(\mu_{\sigma}^{2}\right) \\
& \simeq 9 \lambda_{\sigma}^{2}\left(T \int_{p} \frac{1}{\left[p^{2}+\mu_{\sigma, 3}^{2}\right]} \int_{Q}^{\prime} \frac{1}{Q^{4}}+T \int_{q} \frac{1}{\left[q^{2}+\mu_{\sigma, 3}^{2}\right]^{2}} \int_{P}^{\prime} \frac{1}{P^{2}}\right) \\
& \rightarrow 9 \lambda_{\sigma}(\underbrace{\lambda_{\sigma} T I_{1}^{3}\left(\mu_{\sigma, 3}\right) I_{2}^{4 b}}_{(B)}+\underbrace{\lambda_{\sigma, 3} I_{2}^{3}\left(\mu_{\sigma, 3}\right) I_{1}^{4 b}}_{(C)})
\end{aligned}
$$

where the pure $3 \mathrm{~d}$ vertex is resummed and corresponds to the $3 \mathrm{~d}$ effective one. The mixed mode contributions $(A),(B)$ and $(C)$ are illustrated in figure 5 , together with counterterm interaction diagrams that read

$$
\underbrace{\frac{1}{2} \times 6 \Pi_{\sigma, 4} T I_{1}^{3}\left(\mu_{\sigma, 3}\right)}_{(D)}-\underbrace{\frac{1}{2} \times 6 \lambda_{\sigma, 3} \Pi_{\sigma, 2} T I_{2}^{3}\left(\mu_{\sigma, 3}\right)}_{(E)} .
$$

Consequently, all non-analytic mixed soft/hard contributions vanish in resummation since $(A)+\cdots+(E)=0$. In particular, the IR-sensitive contributions $(C)$ and $(E)$ are $\mathcal{O}\left(g^{3}\right)$ (instead of $\mathcal{O}\left(g^{4}\right)$ ) and IR-divergent in the limit of vanishing $\mu_{\sigma, 3}^{2} \rightarrow 0$. Since dimensional reduction is IR-safe these problematic contributions were expected to vanish.

In practice, the unbroken computation can be conducted by dealing a zero-mass to propagators in two-loop diagrams even though resummation is conceptually indispensable. As a result, IR-divergent contributions vanish in dimensional regularisation which, in turn, obscures the need for explicit resummation.

\subsection{Matching relations for 3d parameters}

The ensuing matching relations define the dimensionally reduced 3d EFT based on the parameters of the fundamental $4 \mathrm{~d}$ theory and temperature. These follow the explicit com- 
putations of the previous sections:

$$
\begin{aligned}
& \mu_{1,3}\left(\Lambda_{3 \mathrm{~d}}\right)=T^{-\frac{1}{2}}\left(\mu_{1}(\Lambda)+\left[\frac{1}{12} T^{2} \mu_{3}(\Lambda)-\frac{L_{b}}{(4 \pi)^{2}} \mu_{3} \mu_{\sigma}^{2}(\Lambda)\right]_{1 \text { loop }}\right. \\
& -\left[\left(\frac{\zeta_{3}}{(4 \pi)^{4}} \frac{2}{3} \frac{\mu_{3}^{2}}{T^{2}}\right)\left(\mu_{1}+\frac{1}{12} T^{2} \mu_{3}\right)\right]_{\mathrm{f.n} .} \\
& \left.+\left[\frac{L_{b}}{(4 \pi)^{2}} \frac{3}{4} T^{2} \lambda_{\sigma} \mu_{3}+\frac{1}{(4 \pi)^{4}} \mu_{3}^{3}\left(3+2 L_{b}+L_{b}^{2}\right)\right]_{2 \mathrm{loop}}\right) \\
& -\left[\frac{1}{(4 \pi)^{2}} 2 \lambda_{\sigma, 3} \mu_{3,3}\left(c+\ln \left(\frac{3 T}{\Lambda_{3 \mathrm{~d}}}\right)\right)\right]_{3 \mathrm{~d} \text {-running }}, \\
& \mu_{\sigma, 3}^{2}\left(\Lambda_{3 \mathrm{~d}}\right)=\mu_{\sigma}^{2}(\Lambda) \\
& +\left[\frac{1}{4} T^{2} \lambda_{\sigma}(\Lambda)-\frac{L_{b}}{(4 \pi)^{2}}\left(2 \mu_{3}^{2}(\Lambda)+3 \lambda_{\sigma} \mu_{\sigma}^{2}(\Lambda)\right)+\frac{\zeta_{3}}{(4 \pi)^{4} T^{2}} 8 \mu_{3}^{2} \mu_{\sigma}^{2}(\Lambda)\right]_{1 \mathrm{loop}} \\
& -\left[\frac{\zeta_{3}}{(4 \pi)^{4} T^{2}} \frac{4}{3} \mu_{3}^{2}\left(\mu_{\sigma}^{2}(\Lambda)+\frac{1}{4} T^{2} \lambda_{\sigma}-\frac{L_{b}}{(4 \pi)^{2}} 2 \mu_{3}^{2}\right)\right]_{\text {f.n. }} \\
& +\left[-T^{2} \frac{L_{b}}{(4 \pi)^{2}} \frac{9}{4} \lambda_{\sigma}^{2}+\frac{1}{(4 \pi)^{4}} \lambda_{\sigma} \mu_{3}^{2}\left(45+3 L_{b}\left(10+L_{b}\right)+2 \zeta_{3}\right)\right. \\
& \left.-\frac{\zeta_{3}}{(4 \pi)^{6} T^{2}} 8\left(3+2 L_{b}\right) \mu_{3}^{4}\right]_{2 \text { loop }}-\left[\frac{1}{(4 \pi)^{2}} 6 \lambda_{\sigma, 3}^{2}\left(c+\ln \left(\frac{3 T}{\Lambda_{3 \mathrm{~d}}}\right)\right)\right]_{3 \mathrm{~d} \text {-running }}, \\
& \mu_{3,3}=T^{\frac{1}{2}}\left(\mu_{3}(\Lambda)-\frac{L_{b}}{(4 \pi)^{2}} 9 \lambda_{\sigma} \mu_{3}+\frac{\zeta_{3}}{(4 \pi)^{4} T^{2}} 2 \mu_{3}^{3}(4-\underbrace{1}_{\text {f.n. }})\right), \\
& \lambda_{\sigma, 3}=T\left(\lambda_{\sigma}(\Lambda)-\frac{L_{b}}{(4 \pi)^{2}} 9 \lambda_{\sigma}^{2}+\frac{\zeta_{3}}{(4 \pi)^{4} T^{2}} \lambda_{\sigma} \mu_{3}^{2}(48-\underbrace{\frac{8}{3}}_{\text {f.n. }})-\frac{\zeta_{5}}{(4 \pi)^{6} T^{4}} 32 \mu_{3}^{4}\right) \text {, }
\end{aligned}
$$

indicating contributions originating from field normalisation (f.n.), one-loop, and two-loop level. Note that the high- $T$ expansion gives rise to NLO terms at one-loop which are $\mu_{\sigma^{-}}^{2}$ proportional. Importantly, we explicitly denoted the $4 \mathrm{~d}$ scale dependence $(\Lambda)$ in terms of which the running of LO terms produce contributions at NLO $\left(\mathcal{O}\left(g^{4}\right)\right)$. In addition, we indicated that the $3 \mathrm{~d}$ tadpole $\mu_{1,3}$ and mass parameter $\mu_{\sigma, 3}^{2}$ run with the $3 \mathrm{~d}$ renormalisation scale $\Lambda_{3 \mathrm{~d}}$. An exact dependence on $\Lambda_{3 \mathrm{~d}}$ is presented even though it includes higher contributions than $\mathcal{O}\left(g^{4}\right)$. This exact dependence can be solved due to the super-renormalisability of the 3d EFT; see section 3.4.

By applying $\beta$-functions of appendix A.1 and ref. [151], we immediately observe that all $3 \mathrm{~d}$ parameters are independent of the $4 \mathrm{~d}$ renormalisation scale $\Lambda$ at $\mathcal{O}\left(g^{4}\right)$ :

$$
\Lambda \frac{\mathrm{d}}{\mathrm{d} \Lambda} \mu_{1,3}=0, \quad \Lambda \frac{\mathrm{d}}{\mathrm{d} \Lambda} \mu_{\sigma, 3}^{2}=0, \quad \Lambda \frac{\mathrm{d}}{\mathrm{d} \Lambda} \mu_{3,3}=0, \quad \Lambda \frac{\mathrm{d}}{\mathrm{d} \Lambda} \lambda_{\sigma, 3}=0 .
$$

For example, the temperature-dependent scale dependence of the $3 \mathrm{~d}$ mass parameter $\mu_{\sigma, 3}^{2}$ in eq. (3.48) arises via its one-loop running contribution $\frac{1}{4} T^{2} \lambda_{\sigma}(\Lambda)$ and cancels upon its two-loop logarithmic term $\propto T^{2} L_{b}$. As a general feature for other scale-dependent terms, this renormalisation scale dependence is discussed in ref. [173]. It is worth to point out that - as depicted in eqs. (3.47)-(3.50) - the tadpole and mass parameter are running 
in terms of the $3 \mathrm{~d}$ renormalisation scale $\Lambda_{3 \mathrm{~d}}$ whereas couplings do not. This dependence of 3d RG scale cancels in computations within the EFT, as we illustrate in the next section.

\subsection{Two-loop effective potential in 3d EFT}

To conclude this section, we illustrate the computation of the two-loop thermal effective potential, within dimensionally reduced $3 \mathrm{~d}$ perturbation theory. This corresponds to step (e) of section 2.3. At two-loop level, the $3 \mathrm{~d}$ effective potential composes of

$$
V_{\text {eff }}^{3 \mathrm{~d}}=V_{\text {tree }}^{3 \mathrm{~d}}+V_{\mathrm{CT}}^{3 \mathrm{~d}}+V_{\text {lloop }}^{3 \mathrm{~d}}+V_{2 \mathrm{loop}}^{3 \mathrm{~d}} .
$$

The $3 \mathrm{~d}$ mass is given by the mass eigenvalue: $m_{3 \mathrm{~d}}^{2}=\mu_{\sigma, 3}^{2}+2 \mu_{3,3} s_{3}+3 \lambda_{\sigma, 3} s_{3}^{2}$, where $s_{3}$ is a background field of the $3 \mathrm{~d}$ theory. The corresponding vertices read

$$
\begin{aligned}
& V_{\sigma^{3}, 3 \mathrm{~d}}=-3 !\left(\frac{\mu_{3,3}}{3}+s_{3} \lambda_{\sigma, 3}\right), \\
& V_{\sigma^{4}, 3 \mathrm{~d}}=-4 !\left(\frac{\lambda_{\sigma, 3}}{4}\right) .
\end{aligned}
$$

The individual pieces of the potential read

$$
\begin{aligned}
V_{\text {tree }}^{3 \mathrm{~d}} & =\frac{1}{2} \mu_{\sigma, 3}^{2}\left(\Lambda_{3 \mathrm{~d}}\right) s_{3}^{2}+\mu_{1,3}\left(\Lambda_{3 \mathrm{~d}}\right) s_{3}^{2}+\frac{1}{3} \mu_{3,3} s_{3}^{3}+\frac{1}{4} \lambda_{\sigma, 3} s_{3}^{4}, \\
V_{\mathrm{CT}}^{3 \mathrm{~d}} & =\delta V_{0}^{3 \mathrm{~d}}+\frac{1}{2} \delta \mu_{\sigma, 3}^{2} s_{3}^{2}+\delta \mu_{1,3} s_{3}, \\
V_{1 \text { loop }}^{\text {3d }} & =J_{\text {soft }}\left(m_{3 \mathrm{~d}}\right), \\
V_{2 \text { loop }}^{3 \mathrm{~d}} & =-\left(\frac{1}{8} V_{\sigma^{4}, 3 \mathrm{~d}}(-1) \mathcal{D}_{S S}^{3 \mathrm{~d}}\left(m_{3 \mathrm{~d}}, m_{3 \mathrm{~d}}\right)+\frac{1}{12}\left(V_{\sigma^{3}, 3 \mathrm{~d}}\right)^{2} \mathcal{D}_{S S S}^{3 \mathrm{~d}}\left(m_{3 \mathrm{~d}}, m_{3 \mathrm{~d}}, m_{3 \mathrm{~d}}\right)\right),
\end{aligned}
$$

with the respective master (loop) integrals collected in appendix B. In the $3 \mathrm{~d}$ EFT divergences stemming from the field dependence appear only at two-loop level wherefore one-loop diagrams with counterterms contribute at three-loop level. The only parameters in need of renormalisation are the mass, tadpole, and field-independent vacuum counterterm. Their counterterms are

$$
\begin{aligned}
\delta \mu_{1,3} & =\frac{1}{(4 \pi)^{2}} \frac{1}{4 \epsilon} 2 \lambda_{\sigma, 3} \mu_{3,3}, \\
\delta \mu_{\sigma, 3}^{2} & =\frac{1}{(4 \pi)^{2}} \frac{1}{4 \epsilon} 6 \lambda_{\sigma, 3}^{2}, \\
\delta V_{0}^{3 \mathrm{~d}} & =\frac{1}{(4 \pi)^{2}} \frac{1}{4 \epsilon} \frac{1}{3} \mu_{3,3}^{2} .
\end{aligned}
$$

The upper two of these counterterms are exact due to super-renormalisability of the 3d EFT; new divergences are absent for tadpole and scalar mass at higher loop orders. The field-independent vacuum counterterm gets contributions up to four-loop order [160]. Hence, we can solve an exact renormalisation scale $\left(\Lambda_{3 \mathrm{~d}}\right)$ dependence of the $3 \mathrm{~d}$ parameters by requiring that the bare parameters are scale-invariant:

$$
\begin{aligned}
& \Lambda_{3 \mathrm{~d}} \frac{\mathrm{d}}{\mathrm{d} \Lambda_{3 \mathrm{~d}}} \mu_{1,3(b)}=\Lambda_{3 \mathrm{~d}} \frac{\mathrm{d}}{\mathrm{d} \Lambda_{3 \mathrm{~d}}}\left(\Lambda_{3 \mathrm{~d}}^{4 \epsilon}\left(\mu_{1,3}\left(\Lambda_{3 \mathrm{~d}}\right)+\delta \mu_{1,3}\right)\right)=0, \\
& \Lambda_{3 \mathrm{~d}} \frac{\mathrm{d}}{\mathrm{d} \Lambda_{3 \mathrm{~d}}} \mu_{\sigma, 3(b)}^{2}=\Lambda_{3 \mathrm{~d}} \frac{\mathrm{d}}{\mathrm{d} \Lambda_{3 \mathrm{~d}}}\left(\Lambda_{3 \mathrm{~d}}^{4 \epsilon}\left(\mu_{\sigma, 3}^{2}\left(\Lambda_{3 \mathrm{~d}}\right)+\delta \mu_{\sigma, 3}^{2}\right)\right)=0 .
\end{aligned}
$$


Their solution yields

$$
\begin{aligned}
& \mu_{1,3}\left(\Lambda_{3 \mathrm{~d}}\right)=-\frac{1}{(4 \pi)^{2}} 2 \lambda_{\sigma, 3} \mu_{3,3} \ln \left(\frac{\Lambda_{0}}{\Lambda_{3 \mathrm{~d}}}\right)+\mathcal{C}_{1}, \\
& \mu_{\sigma, 3}^{2}\left(\Lambda_{3 \mathrm{~d}}\right)=-\frac{1}{(4 \pi)^{2}} 6 \lambda_{\sigma, 3}^{2} \ln \left(\frac{\Lambda_{0}}{\Lambda_{3 \mathrm{~d}}}\right)+\mathcal{C}_{2},
\end{aligned}
$$

where temperature-dependent initial conditions are parametrised by $\Lambda_{0} \equiv 3 T e^{c}$ and coefficients $\mathcal{C}_{1,2}$ are fixed to reproduce the $\mathcal{O}\left(g^{4}\right)$ hard mode contributions of the matching relations at $\Lambda_{3 \mathrm{~d}}=\Lambda_{4 \mathrm{~d}}$. The above renormalised parameters $\mu_{3,3}$ in eq. (3.49) and $\lambda_{\sigma, 3}$ in eq. (3.50) are RG-invariant. In total, the effective potential at two-loop has a compact result

$$
\begin{aligned}
V_{\mathrm{eff}}^{3 \mathrm{~d}}= & \frac{1}{2} \mu_{\sigma, 3}^{2}\left(\Lambda_{3 \mathrm{~d}}\right) s_{3}^{2}+\mu_{1,3}\left(\Lambda_{3 \mathrm{~d}}\right) s_{3}+\frac{1}{3} \mu_{3,3} s_{3}^{3}+\frac{1}{4} \lambda_{\sigma, 3} s_{3}^{4}-\frac{\left(m_{3 \mathrm{~d}}^{2}\right)^{\frac{3}{2}}}{12 \pi} \\
& +\frac{1}{(4 \pi)^{2}}\left(\frac{3}{4} \lambda_{\sigma, 3} m_{3 \mathrm{~d}}^{2}-\frac{1}{6}\left(3 \lambda_{\sigma, 3} s_{3 \mathrm{~d}}+\mu_{3,3}\right)^{2}\left[1+2 \ln \left(\frac{\Lambda_{3 \mathrm{~d}}}{3 m_{3 \mathrm{~d}}}\right)\right]\right) .
\end{aligned}
$$

We observe that the tree-level running of the $3 \mathrm{~d}$ parameters compensates the scale dependence in the two-loop logarithmic terms. This $R G$-improved effective potential (and even the three-loop effective potential) is compared to non-perturbative lattice simulations in ref. [160]. The latter agrees surprisingly well even with large expansion parameters in perturbation theory.

This concludes our instructions to the dimensional reduction of the real scalar field theory.

\section{Dimensional reduction of the real-singlet extended Standard Model}

This section details the dimensionally reduced 3d EFT for the SM coupled to a real scalar singlet at NLO. This is the novel result of this article. Applications of this 3d EFT to study of electroweak phase transition in the xSM are presented in refs. [172, 173].

When extending the Standard Model by a real scalar singlet, the position of that scalar in the high-temperature hierarchy is a priori undetermined. If the scalar singlet assumes a hard (or "superheavy") scale it is integrated out entirely during the dimensional reduction [151]. ${ }^{6}$ The resulting version of the SM 3d EFT encodes effects of the singlet merely in its matching relations.

The following analysis relaxes this assumption and performs the dimensional reduction with a soft (or "heavy") singlet. The singlet remains a dynamical field in the $3 \mathrm{~d}$ EFT and can eventually become ultrasoft (or "light"). Such a configuration allows for dynamical transitions with two consecutive steps which are a viable candidate for EWPT with SFOPT in this model (cf. singlet refs. in section 1). During such a dynamical two-step transition, first the singlet acquires a non-zero vacuum expectation value (vev) at high temperatures which is followed by a SFOPT in Higgs-direction once temperature is lowered further.

\footnotetext{
${ }^{6}$ However, ref. [151] lacks full $\mathcal{O}\left(g^{4}\right)$ accuracy since the Higgs 3d mass parameter at two-loop order is not computed.
} 
The scalar sector of the $4 \mathrm{~d}$ Lagrangian reads

$$
\begin{aligned}
\mathcal{L}_{\text {scalar }}^{4 \mathrm{~d}}= & \left(D_{\mu} \phi\right)^{\dagger}\left(D_{\mu} \phi\right)+\mu_{h}^{2} \phi^{\dagger} \phi+\lambda_{h}\left(\phi^{\dagger} \phi\right)^{2} \\
& +\frac{1}{2}\left(\partial_{\mu} \sigma\right)^{2}+\frac{1}{2} \mu_{\sigma}^{2} \sigma^{2}+\mu_{1} \sigma+\frac{1}{3} \mu_{3} \sigma^{3}+\frac{1}{4} \lambda_{\sigma} \sigma^{4} \\
& +\frac{1}{2} \mu_{m} \sigma \phi^{\dagger} \phi+\frac{1}{2} \lambda_{m} \sigma^{2} \phi^{\dagger} \phi
\end{aligned}
$$

which notationally aligns with ref. [151] (see section 2 ibid.) except the opposite sign convention for the $4 \mathrm{~d}$ Higgs mass parameter $\mu_{h}^{2}$.

We assume the following formal power counting, or scaling in powers of the $\mathrm{SU}(2)$ gauge coupling $g$ :

$$
g^{\prime}, g_{\mathrm{s}}, g_{Y} \sim g, \quad \lambda_{h}, \lambda_{\sigma}, \lambda_{m} \sim g^{2}, \quad \mu_{h}^{2}, \mu_{\sigma}^{2} \sim(g T)^{2}
$$

Since both mass parameters are soft, this leads to an EFT with two dynamical light scalars $\phi_{3}$ and $\sigma_{3}$. The scaling of dimensionful couplings is more delicate (cf. section 2.1.2 in ref. [151]), where for the tadpole and cubic couplings we assume

$$
\mu_{1} \sim g T^{3}, \quad \mu_{m}, \mu_{3} \sim g T .
$$

In analogy to eq. (3.3) this formal choice leads to similar peculiarities. Contributions

$$
\begin{aligned}
& =\quad \simeq \frac{\mu_{m}^{2}}{\mu_{\sigma}^{2}} \sim \mathcal{O}(1) \gg \lambda_{h}, \\
& =\frac{\mu_{m} \mu_{3}}{\mu_{\sigma}^{2}} \sim \mathcal{O}(1) \gg \lambda_{m},
\end{aligned}
$$

parametrically dominate over the corresponding quartic couplings. We reiterate the strategy below eq. (3.3): for generality we install $\mu_{m}, \mu_{3} \sim g T$ and include all contributions of cubic couplings in our matching relations. Note, that contributions proportional to $\mu_{3}^{2}$ and $\mu_{3}^{4}$ are further numerically suppressed by extra powers of $1 /(4 \pi)$. In the chosen formal scaling, the one-loop contributions of the tadpole correlator $\sim \mu_{m, 3} \times T^{2}$ are formally of the same order as the tree-level tadpole. Therefore, the $\beta$-functions of tadpole and mass parameters (cf. appendix A.1), are partly needed at two-loop level for a $\mathcal{O}\left(g^{4}\right)$ accuracy.

\subsection{Effective 3d theories}

The corresponding effective 3d Lagrangian is

$$
\begin{aligned}
\mathcal{L}_{\text {scalar }}^{\text {3d }}= & \left(D_{r} \phi\right)^{\dagger}\left(D_{r} \phi\right)+\mu_{h, 3}^{2} \phi^{\dagger} \phi+\lambda_{h, 3}\left(\phi^{\dagger} \phi\right)^{2} \\
& +\frac{1}{2}\left(\partial_{r} \sigma\right)^{2}+\frac{1}{2} \mu_{\sigma, 3}^{2} \sigma^{2}+\mu_{1,3} \sigma+\frac{1}{3} \mu_{3,3} \sigma^{3}+\frac{1}{4} \lambda_{\sigma, 3} \sigma^{4} \\
& +\frac{1}{2} \mu_{m, 3} \sigma \phi^{\dagger} \phi+\frac{1}{2} \lambda_{m, 3} \sigma^{2} \phi^{\dagger} \phi
\end{aligned}
$$


with $r \in\{1, \ldots, d\}$ and the 3 -subscript denoting parameters in the dimensionally reduced 3d EFT. ${ }^{7}$ In addition, we include the following (non-kinetic) pure scalar marginal operators

$$
\begin{aligned}
\mathcal{L}_{\text {marginal }}^{3 \mathrm{~d}}= & c_{0,5} \sigma^{5}+c_{2,3}\left(\phi^{\dagger} \phi\right) \sigma^{3}+c_{4,1}\left(\phi^{\dagger} \phi\right)^{2} \sigma \\
& +c_{6,0}\left(\phi^{\dagger} \phi\right)^{3}+c_{0,6} \sigma^{6}+c_{4,2}\left(\phi^{\dagger} \phi\right)^{2} \sigma^{2}+c_{2,4}\left(\phi^{\dagger} \phi\right) \sigma^{4} .
\end{aligned}
$$

The nomenclature of the effective field theory [232, 234, 235] classifies these operators as $S^{6}$. We omit classes with higher dimensional kinetic operators such as $D^{2} S^{4}$ and $D^{4} S^{2}$ where $D$ formally presents a derivative operator and classes with gauge fields such as $F^{3}$ where $F$ presents a field strength tensor. This choice is purely practical: the matching of class $S^{6}$ is straightforward since corresponding correlators can be computed at zero external momenta both from the effective potential in the broken phase and even diagrammatically directly in unbroken phase. On the other hand, the derivative structure of kinetic operators requires a computation with explicit external momenta dependence. We defer this challenge to a future comprehensive analysis of the numerical relevance of different higher dimensional operators. However, in the presence of large portal couplings it is natural to expect the class $S^{6}$ to numerically dominate over other classes that are always suppressed by $g^{2}$.

The breaking of Lorentz symmetry by the heat bath induces temporal scalars. These are remnants of the temporal gauge field components [212] and obtain Debye screening masses at the soft scale. In analogy with "electrostatic" QCD [113], the Lagrangian composes of

$$
\begin{aligned}
\mathcal{L}_{\text {temporal }}^{3 \mathrm{~d}}= & \frac{1}{2}\left(D_{r} A_{0}^{a}\right)^{2}+\frac{1}{2} m_{\mathrm{D}}^{2} A_{0}^{a} A_{0}^{a}+\frac{1}{2}\left(\partial_{r} B_{0}\right)^{2}+\frac{1}{2} m_{\mathrm{D}}^{\prime 2} B_{0}^{2}+\frac{1}{2}\left(D_{r} C_{0}^{\alpha}\right)^{2}+\frac{1}{2} m_{\mathrm{D}}^{\prime \prime 2} C_{0}^{\alpha} C_{0}^{\alpha} \\
& +\frac{1}{4} \kappa_{3}\left(A_{0}^{a} A_{0}^{a}\right)^{2}+\frac{1}{4} \kappa_{3}^{\prime} B_{0}^{4}+\frac{1}{4} \kappa_{3}^{\prime \prime} A_{0}^{a} A_{0}^{a} B_{0}^{2} \\
& +h_{3} \phi^{\dagger} \phi A_{0}^{a} A_{0}^{a}+h_{3}^{\prime} \phi^{\dagger} \phi B_{0}^{2}+h_{3}^{\prime \prime} B_{0} \phi^{\dagger} A_{0}^{a} \tau^{a} \phi+\delta_{3} \phi^{\dagger} \phi C_{0}^{\alpha} C_{0}^{\alpha} \\
& +x_{3} \sigma A_{0}^{a} A_{0}^{a}+x_{3}^{\prime} \sigma B_{0}^{2}+y_{3} \sigma^{2} A_{0}^{a} A_{0}^{a}+y_{3}^{\prime} \sigma^{2} B_{0}^{2}
\end{aligned}
$$

wherein $\tau^{a}$ denote the Pauli matrices and the covariant derivatives act on the adjoint scalars as $D_{r} A_{0}^{a}=\partial_{r} A_{0}^{a}+g_{3} \epsilon_{b c}^{a} A_{r}^{b} A_{0}^{c}$ and $D_{r} C_{0}^{\alpha}=\partial_{r} C_{0}^{\alpha}+g_{\mathrm{s}, 3} f_{\beta \rho}^{\alpha} C_{r}^{\beta} C_{0}^{\rho}$.

Several interaction terms among adjoint scalars were omitted in the temporal Lagrangian since they are of secondary interest in our computation [151]. Among these omissions are operators with an odd number of temporal fields such as $\sigma \phi^{\dagger} A_{0}^{a} \tau^{a} \phi$. These only appear in the presence of a finite chemical potential due to the breaking of parity [127]. Exceeding the accuracy of our analysis, we exclude higher dimensional operators involving temporal scalars since they are numerically suppressed compared to large scalar portal couplings [111]. Besides, the effect of the temporal sector is numerically subdominant in strong phase transitions driven by ultrasoft (light) scalar fields. This suppression is often empirically observed in BSM theories since physically the temporal scalars are screened at length scales much shorter than those relevant to the phase transition dynamics.

At the ultrasoft scale, the dynamics of the soft (heavy) temporal scalars $A_{0}^{a}, B_{0}$ and $C_{0}^{\alpha}$ has been integrated out by the second step of dimensional reduction [111]. Remember that

\footnotetext{
${ }^{7}$ For simplicity, we keep the notation for 3-dimensional fields identical as in the fundamental $4 \mathrm{~d}$ theory.
} 
their masses are at the soft scale and are not dynamical in the vicinity of the transition. The resulting Lagrangian resembles eqs. (4.6) and (4.7) but all parameters are denoted with a bar.

\subsection{Integrating out the hard scale}

The first step of the dimensional reduction occurs from hard to soft scale by integrating out all hard, non-zero Matsubara modes. Here, the dimensional reduction is performed at NLO $\left(\mathcal{O}\left(g^{4}\right)\right)$, which means at one-loop in the couplings, two-loop in the tadpole and masses, and one-loop in the field renormalisations. This section merely quotes final results and matching relations, casting details of the computation of correlators to appendix A. We employ a general covariant gauge with gauge parameters $\xi_{1}$ for $\mathrm{U}(1)_{\mathrm{Y}}$, and $\xi_{2}$ for $\mathrm{SU}(2)$ opposed to Landau gauge in ref. [151].

Normalisation of fields. The relations between $4 \mathrm{~d}$ and $3 \mathrm{~d}$ scalar fields are (for generic field $\psi$ )

$$
\left(\psi^{2}\right)_{3 \mathrm{~d}}=\frac{\left(\psi^{2}\right)_{4 \mathrm{~d}}}{T}\left[1+\hat{\Pi}_{\psi^{2}}^{\prime}\right],
$$

where primed correlators are differentiated with respect to the external momentum squared. The hat denotes the correlator in renormalised perturbation theory with implicit counterterms $\delta Z_{\psi}$ in appendix A.1. The corresponding renormalised 2-point correlation functions yield

$$
\begin{aligned}
\hat{\Pi}_{A_{0}^{a} A_{0}^{b}}^{\prime} & =\frac{g^{2}}{(4 \pi)^{2}}\left(3+\frac{\left(N_{\mathrm{c}}+1\right)}{3} n_{\mathrm{f}}\left(L_{f}-1\right)+\left(\xi_{2}-\frac{25}{6}\right) L_{b}-2 \xi_{2}\right), \\
\hat{\Pi}_{A_{r}^{a} A_{s}^{b}}^{\prime} & =\frac{g^{2}}{(4 \pi)^{2}}\left(-\frac{2}{3}+\frac{\left(N_{\mathrm{c}}+1\right)}{3} n_{\mathrm{f}} L_{f}+\left(\xi_{2}-\frac{25}{6}\right) L_{b}\right), \\
\hat{\Pi}_{B_{0} B_{0}}^{\prime} & =\frac{g^{\prime 2}}{(4 \pi)^{2}} \frac{1}{6}\left(Y_{\phi}^{2}\left(L_{b}+2\right)+Y_{2 \mathrm{f}} n_{\mathrm{f}}\left(L_{f}-1\right)\right), \\
\hat{\Pi}_{B_{r} B_{s}}^{\prime} & =\frac{g^{\prime 2}}{(4 \pi)^{2}} \frac{1}{6}\left(Y_{\phi}^{2} L_{b}+Y_{2 \mathrm{f}} n_{\mathrm{f}} L_{f}\right), \\
\hat{\Pi}_{\phi^{\dagger} \phi}^{\prime} & =\frac{1}{(4 \pi)^{2}}\left(-\frac{L_{b}}{4}\left(3\left(3-\xi_{2}\right) g^{2}+\left(3-\xi_{1}\right) g^{\prime 2}\right)+N_{\mathrm{c}} L_{f} g_{Y}^{2}\right)+\frac{\zeta_{3}}{(4 \pi)^{4}} \frac{1}{6} \frac{\mu_{m}^{2}}{T^{2}}, \\
\hat{\Pi}_{\sigma \sigma}^{\prime} & =\frac{\zeta_{3}}{(4 \pi)^{4} T^{2}} \frac{1}{3}\left(4 \mu_{3}^{2}+\mu_{m}^{2}\right),
\end{aligned}
$$

where $n_{\mathrm{f}}=3$ is the number of quark and lepton families and $N_{\mathrm{c}}=3$ the number of colours. The $\mathrm{U}(1)_{\mathrm{Y}}$ hypercharges are

$$
Y_{\ell}=-1, \quad Y_{e}=-2, \quad Y_{q}=\frac{1}{3}, \quad Y_{u}=\frac{4}{3}, \quad Y_{d}=-\frac{2}{3}, \quad Y_{\phi}=1
$$

for which we abbreviate recurring sums as

$$
\begin{aligned}
& \sum_{f} Y_{\mathrm{f}}^{4} \equiv Y_{4 \mathrm{f}}=\left[\left(Y_{e}^{4}+2 Y_{\ell}^{4}\right)+N_{\mathrm{c}}\left(Y_{u}^{4}+Y_{d}^{4}+2 Y_{q}^{4}\right)\right]=\frac{2}{81}\left(729+137 N_{\mathrm{c}}\right)=\frac{760}{27}, \\
& \sum_{f} Y_{\mathrm{f}}^{2} \equiv Y_{2 \mathrm{f}}=\left[\left(Y_{e}^{2}+2 Y_{\ell}^{2}\right)+N_{\mathrm{c}}\left(Y_{u}^{2}+Y_{d}^{2}+2 Y_{q}^{2}\right)\right]=\frac{2}{9}\left(27+11 N_{\mathrm{c}}\right)=\frac{40}{3} .
\end{aligned}
$$


The matching between the $4 \mathrm{~d}$ and effective $3 \mathrm{~d}$ theory relates their couplings at one-loop order

$$
\begin{aligned}
\lambda_{h, 3} & =T\left(\lambda_{h}+\frac{1}{2} \hat{\Gamma}_{\left(\phi^{\dagger} \phi\right)^{2}}-2 \lambda_{h} \hat{\Pi}_{\phi^{\dagger} \phi}^{\prime}\right) \\
\mu_{3,3} & =T^{\frac{1}{2}}\left(\mu_{3}+\frac{1}{2} \hat{\Gamma}_{\sigma^{3}}-\frac{3}{2} \mu_{3} \hat{\Pi}_{\sigma \sigma}^{\prime}\right) \\
\lambda_{\sigma, 3} & =T\left(\lambda_{\sigma}+\frac{1}{6} \hat{\Gamma}_{\sigma^{4}}-2 \lambda_{\sigma} \hat{\Pi}_{\phi^{\dagger} \phi}^{\prime}\right) \\
\mu_{m, 3} & =T^{\frac{1}{2}}\left(\mu_{m}+2 \hat{\Gamma}_{\phi^{\dagger} \phi \sigma}-\mu_{m}\left(\hat{\Pi}_{\phi^{\dagger} \phi}^{\prime}+\frac{1}{2} \hat{\Pi}_{\sigma \sigma}^{\prime}\right)\right), \\
\lambda_{m, 3} & =T\left(\lambda_{m}+\hat{\Gamma}_{\phi^{\dagger} \phi \sigma^{2}}-\lambda_{m}\left(\hat{\Pi}_{\phi^{\dagger} \phi}^{\prime}+\hat{\Pi}_{\sigma \sigma}^{\prime}\right)\right) \\
x_{3} & =\frac{1}{2} T \hat{\Gamma}_{A_{0}^{a} A_{0}^{b} \sigma}, \\
x_{3}^{\prime} & =\frac{1}{2} T \hat{\Gamma}_{B_{0} B_{0} \sigma}, \\
y_{3} & =\frac{1}{4} T \hat{\Gamma}_{A_{0}^{a} A_{0}^{b} \sigma^{2}}, \\
y_{3}^{\prime} & =\frac{1}{4} T \hat{\Gamma}_{B_{0} B_{0} \sigma^{2}},
\end{aligned}
$$

where $\hat{\Gamma}$ represents a $n$-point correlation function with subscript corresponding to external fields. The mass parameters and tadpole at two-loop match according to

$$
\begin{aligned}
& \mu_{h, 3}^{2}=\mu_{h}^{2}+\hat{\Pi}_{\phi^{\dagger} \phi}^{1 \text { loop }}-\left(\mu_{h}^{2}+\hat{\Pi}_{\phi^{\dagger} \phi}^{1 \text { loop }}\right) \hat{\Pi}_{\phi^{\dagger} \phi}^{\prime}+\hat{\Pi}_{\phi^{\dagger} \phi}^{2 \text { loop }}, \\
& \mu_{\sigma, 3}^{2}=\mu_{\sigma}^{2}+\hat{\Pi}_{\sigma \sigma}^{1 \text { loop }}-\left(\mu_{\sigma}^{2}+\hat{\Pi}_{\sigma \sigma}^{1 \text { loop }}\right) \hat{\Pi}_{\sigma \sigma}^{\prime}+\hat{\Pi}_{\sigma \sigma}^{\text {2loop }}, \\
& \mu_{1,3}=T^{\frac{1}{2}}\left(\mu_{1}+\hat{\Gamma}_{\sigma}^{1 \text { loop }}-\frac{1}{2}\left(\mu_{1}+\hat{\Gamma}_{\sigma}^{1 \text { loop }}\right) \hat{\Pi}_{\sigma \sigma}^{\prime}+\hat{\Gamma}_{\sigma}^{2 \text { loop }}\right) .
\end{aligned}
$$

The matching of marginal operators (4.7) is analogous and we do not explicate their formulas. ${ }^{8}$ We showcase the computation of the required correlators in appendix A.4. As an example, for the quartic Higgs coupling, we obtain

$$
\begin{aligned}
\lambda_{h, 3}=T & {[\underbrace{\lambda_{h}(\Lambda)}_{\mathcal{O}\left(g^{4}\right) \text { running }}+\frac{1}{(4 \pi)^{2}}\left(\frac{2-3 L_{b}}{16}\left(3 g^{4}+2 g^{2} g^{\prime 2}+g^{\prime 4}\right)+N_{\mathrm{c}} g_{Y}^{2} L_{f}(g_{Y}^{2}-\underbrace{2 \lambda_{h}}_{\text {f.n. }})\right.} \\
& +L_{b}(\underbrace{\frac{3}{2}\left(3 g^{2}+g^{\prime 2}\right) \lambda_{h}}_{\text {f.n. }}-12 \lambda_{h}^{2}-\frac{1}{4} \lambda_{m})-\frac{1}{2}(\underbrace{\left.1-1) L_{b} \lambda_{h}\left(3 g^{3} \xi_{2}+g^{\prime 2} \xi_{1}\right)\right)}_{\text {f.n. }} \\
& \left.+\frac{\zeta_{3}}{(4 \pi)^{4} T^{2}}\left((3-\underbrace{\frac{1}{3}}_{\text {f.n. }}) \lambda_{h}+\frac{1}{2} \lambda_{m}\right) \mu_{m}^{2}-\frac{\zeta_{5}}{(4 \pi)^{6} T^{4}} \frac{\mu_{m}^{4}}{4}\right],
\end{aligned}
$$

where we indicated the origin of individual contributions, displaying cancellations between correlators and field normalisation (f.n.) for the $R G$-scale and gauge dependence. To witness

\footnotetext{
${ }^{8}$ In fact, matching relations of marginal operators are simpler since for them field normalisations are of even higher order and thus absent.
} 
a cancellation of the RG-scale $\Lambda$ at $\mathcal{O}\left(g^{4}\right)$ order, we include the one-loop $\beta$-function (A.8) for the tree-level piece, which cancels the logarithmic scale dependence in $L_{b, f}$. In these cancellations, the contribution from field normalisation is essential. Note that the coefficient of $\ln (\Lambda / T)$ (in $L_{b, f}$ ) matches the $\beta$-function for the tree-level parameter.

The matching of other parameters yields analogously

$$
\begin{aligned}
& \mu_{3,3}=T^{\frac{1}{2}} {\left[\mu_{3}(\Lambda)-\frac{3}{(4 \pi)^{2}} L_{b}\left(3 \lambda_{\sigma} \mu_{3}+\frac{1}{2} \lambda_{m} \mu_{m}\right)+\frac{\zeta_{3}}{(4 \pi)^{4} T^{2}}\left(6 \mu_{3}^{3}-\frac{1}{2} \mu_{3} \mu_{m}^{2}+\frac{1}{2} \mu_{m}^{3}\right)\right], } \\
& \lambda_{\sigma, 3}=T {\left[\lambda_{\sigma}(\Lambda)-\frac{1}{(4 \pi)^{2}} L_{b}\left(\lambda_{m}^{2}+9 \lambda_{\sigma}^{2}\right)+\frac{\zeta_{3}}{(4 \pi)^{4} T^{2}}\left(\frac{136}{3} \lambda_{\sigma} \mu_{3}^{2}+2 \lambda_{m} \mu_{m}^{2}-\frac{2}{3} \lambda_{\sigma} \mu_{m}^{2}\right)\right.} \\
&\left.\quad-\frac{\zeta_{5}}{(4 \pi)^{6} T^{4}}\left(32 \mu_{3}^{4}+\frac{1}{2} \mu_{m}^{4}\right)\right], \\
& \mu_{m, 3}=T^{\frac{1}{2}}\left[\mu_{m}(\Lambda)+\frac{1}{(4 \pi)^{2}}\left(L_{b}\left(\left(\frac{3}{4}\left(3 g^{2}+g^{\prime 2}\right)-6 \lambda_{h}-2 \lambda_{m}\right) \mu_{m}-2 \lambda_{m} \mu_{3}\right)-N_{\mathrm{c}} L_{f} g_{Y}^{2} \mu_{m}\right)\right. \\
&\left.\quad+\frac{\zeta_{3}}{(4 \pi)^{4} T^{2}} \mu_{m}\left(-\frac{2}{3} \mu_{3}^{2}+2 \mu_{3} \mu_{m}+\frac{1}{6} \mu_{m}^{2}\right)\right] .
\end{aligned}
$$

We observe an important - and bluntly surprising - exception for the $Z_{2}$-symmetric Higgs-singlet portal coupling

$$
\begin{aligned}
\lambda_{m, 3}=T[ & \lambda_{m}(\Lambda)+\frac{\lambda_{m}}{(4 \pi)^{2}}\left(L_{b}\left(\frac{3}{4}\left(3 g^{2}+g^{\prime 2}\right)-6 \lambda_{h}-2 \lambda_{m}-3 \lambda_{\sigma}\right)-N_{\mathrm{c}} L_{f} g_{Y}^{2}\right) \\
& +\frac{\zeta_{3}}{(4 \pi)^{4} T^{2}}\left(\lambda_{m}\left(\frac{20}{3} \mu_{3}^{2}+8 \mu_{3} \mu_{m}\right)+\left(6 \lambda_{h}+2 \lambda_{m}+3 \lambda_{\sigma}\right) \mu_{m}^{2}\right) \\
& -\frac{\zeta_{5}}{(4 \pi)^{6} T^{4}} \mu_{m}^{2}\left(8 \mu_{3}^{2}+2 \mu_{3} \mu_{m}+\frac{1}{2} \mu_{m}^{2}\right)-\frac{\zeta_{3}}{(4 \pi)^{4} T^{2}} \mu_{m}^{2} \frac{1}{4} \underbrace{\left(3 g^{2} \xi_{2}+g^{\prime 2} \xi_{1}\right)}_{\xi-\text { dependent }}] .
\end{aligned}
$$

The remaining gauge dependence in $\lambda_{m, 3}$ originates from the correlator $\hat{\Gamma}_{\phi^{\dagger} \phi \sigma^{2}}$ (cf. appendix A.3) and is uncancelled by the field renormalisation contribution which is proportional to the portal coupling $\lambda_{m}$ instead. Since the matched parameters are merely $3 \mathrm{~d}$ effective Lagrangian parameters, they are not directly associated with physical observables of the $3 \mathrm{~d}$ theory and may well depend on the gauge fixing of the $4 \mathrm{~d}$ theory. A similar discussion in ref. [225] addresses the role of higher dimensional operators in hot QCD. We defer the topic of gauge dependence in matching of hot electroweak theories to future research. Meanwhile, an immediate solution (used in ref. [172]) is provided by a stricter power counting $\mu_{m} \sim g^{2} T$ for the cubic portal coupling. Hence, by sticking to $\mathcal{O}\left(g^{4}\right)$, all gauge-dependent contributions are cast to higher orders. 
The singlet interacts with temporal scalars through the couplings

$$
\begin{aligned}
& x_{3}=T \frac{1}{(4 \pi)^{2}} g^{2} \mu_{m}, \\
& x_{3}^{\prime}=T \frac{1}{(4 \pi)^{2}} g^{\prime 2} \mu_{m}, \\
& y_{3}=T\left(\frac{1}{(4 \pi)^{2}} \frac{1}{2} g^{2} \lambda_{m}-\frac{\zeta_{3}}{(4 \pi)^{4} T^{2}} \frac{1}{2} g^{2} \mu_{m}^{2}\right), \\
& y_{3}^{\prime}=T\left(\frac{1}{(4 \pi)^{2}} \frac{1}{2} g^{\prime 2} \lambda_{m}-\frac{\zeta_{3}}{(4 \pi)^{4} T^{2}} \frac{1}{2} g^{\prime 2} \mu_{m}^{2}\right) .
\end{aligned}
$$

The results for the two-loop mass parameters and tadpole coupling read

$$
\begin{aligned}
& \mu_{h, 3}^{2}=\left(\mu_{h, 3}^{2}\right)_{\mathrm{SM}}+\frac{T^{2}}{24} \lambda_{m}(\Lambda) \\
& -\frac{L_{b}}{(4 \pi)^{2}}\left(\frac{1}{4} \mu_{m}^{2}(\Lambda)+\frac{1}{2} \lambda_{m} \mu_{\sigma}^{2}(\Lambda)\right)+\frac{\zeta_{3}}{(4 \pi)^{4}} \frac{\mu_{m}^{2}}{T^{2}} \frac{1}{2}\left(\mu_{h}^{2}(\Lambda)+\mu_{\sigma}^{2}(\Lambda)\right) \\
& -\frac{\zeta_{3}}{6(4 \pi)^{4}} \frac{\mu_{m}^{2}}{T^{2}}\left(\mu_{h}^{2}(\Lambda)+\frac{T^{2}}{12}\left(\frac{3}{4}\left(3 g^{2}+g^{\prime 2}\right)+N_{\mathrm{c}} g_{Y}^{2}+6 \lambda_{h}+\frac{1}{2} \lambda_{m}\right)-\frac{1}{(4 \pi)^{2}} \frac{1}{4} L_{b} \mu_{m}^{2}(\Lambda)\right) \\
& +\frac{1}{(4 \pi)^{2}}\left(\frac{3}{4}\left(3 g^{2}+g^{\prime 2}\right) L_{b}-N_{\mathrm{c}} g_{Y}^{2} L_{f}\right)\left(\frac{T^{2}}{24} \lambda_{m}-\frac{1}{(4 \pi)^{2}} \frac{1}{4} L_{b} \mu_{m}^{2}(\Lambda)\right) \\
& +\frac{1}{(4 \pi)^{4}}\left[\frac{\left(3+2 L_{b}+L_{b}^{2}\right)}{2}\left(\lambda_{m}\left(\mu_{3}^{2}+\mu_{3} \mu_{m}\right)+\frac{9}{2} \mu_{m}^{2} \lambda_{h}\right)\right. \\
& \left.-L_{b}^{2} \mu_{m}^{2}\left(\frac{3}{32}\left(3 g^{2}+g^{\prime 2}\right)-\frac{5}{8} \lambda_{m}\right)+N_{\mathrm{c}} \mu_{m}^{2} g_{Y}^{2} \frac{L_{f}}{4}\left(L_{b}-\frac{1}{2} L_{f}\right)\right] \\
& -\frac{T^{2}}{(4 \pi)^{2}} L_{b} \lambda_{m}\left(\frac{1}{4} \lambda_{h}+\frac{5}{24} \lambda_{m}+\frac{1}{8} \lambda_{\sigma}\right)-\frac{1}{(4 \pi)^{2}} \frac{1}{2} \lambda_{m, 3}^{2}\left(c+\ln \left(\frac{3 T}{\Lambda_{3 \mathrm{~d}}}\right)\right) \\
& -\frac{1}{(4 \pi)^{4}} \frac{\left(3+2 L_{b}\right)}{8} \mu_{m}^{2}\left(\frac{1}{4}\left(3 g^{2}+g^{\prime 2}\right)-N_{\mathrm{c}} g_{Y}^{2}-3 \lambda_{m}\right) \\
& +\frac{\zeta_{3}}{(4 \pi)^{4}} \mu_{m}^{2}\left(\frac{1}{32}\left(3 g^{2}+g^{\prime 2}\right)+\frac{1}{4} \lambda_{h}+\frac{5}{48} \lambda_{m}+\frac{1}{8} \lambda_{\sigma}+\frac{N_{\mathrm{c}}}{24} g_{Y}^{2}\right) \\
& -\frac{\zeta_{3}}{(4 \pi)^{6} T^{2}} \frac{\left(3+2 L_{b}\right)}{16} \mu_{m}^{2}\left(8 \mu_{3}^{2}+3 \mu_{m}^{2}\right) \\
& \mu_{\sigma, 3}^{2}=\mu_{\sigma}^{2}(\Lambda)+T^{2}\left(\frac{1}{6} \lambda_{m}(\Lambda)+\frac{1}{4} \lambda_{\sigma}(\Lambda)\right)+\frac{1}{(4 \pi)^{4}} \frac{\zeta_{3}}{T^{2}}\left(2 \mu_{m}^{2} \mu_{h}^{2}(\Lambda)+8 \mu_{3}^{2} \mu_{\sigma}^{2}(\Lambda)\right) \\
& -\frac{L_{b}}{(4 \pi)^{2}}\left(2 \mu_{3}^{2}(\Lambda)+\frac{1}{2} \mu_{m}^{2}(\Lambda)+2 \lambda_{m} \mu_{h}^{2}(\Lambda)+3 \lambda_{\sigma} \mu_{\sigma}^{2}(\Lambda)\right) \\
& -\frac{\zeta_{3}}{(4 \pi)^{4} T^{2}} \frac{2}{3}\left(2 \mu_{3}^{2}+\frac{1}{2} \mu_{m}^{2}\right)\left(\mu_{\sigma}^{2}(\Lambda)+T^{2}\left(\frac{1}{6} \lambda_{m}+\frac{1}{4} \lambda_{\sigma}\right)-\frac{L_{b}}{(4 \pi)^{2}}\left(2 \mu_{3}^{2}+\frac{1}{2} \mu_{m}^{2}\right)\right) \\
& +\frac{1}{(4 \pi)^{4}}\left[\frac{\left(3+2 L_{b}\right)}{2}\left(30 \lambda_{\sigma} \mu_{3}^{2}+4 \lambda_{m} \mu_{3} \mu_{m}-\mu_{m}^{2}\left(\left(3 g^{2}+g^{\prime 2}\right)-N_{\mathrm{c}} g_{Y}^{2}\right)\right)\right. \\
& +\frac{\left(3+2 L_{b}+L_{b}^{2}\right)}{4} \mu_{m}^{2}\left(5 \lambda_{m}+3 \lambda_{\sigma}\right)+N_{\mathrm{c}} g_{Y}^{2} \mu_{m}^{2} L_{f}\left(L_{b}-\frac{1}{2} L_{f}\right) \\
& \left.+L_{b}^{2}\left(21 \lambda_{\sigma} \mu_{3}^{2}+4 \lambda_{m} \mu_{3} \mu_{m}+3 \lambda_{h} \mu_{m}^{2}-\frac{3}{8}\left(3 g^{2}+g^{\prime 2}\right) \mu_{m}^{2}\right)\right]
\end{aligned}
$$




$$
\begin{aligned}
& +\frac{1}{(4 \pi)^{2}}\left(\left(3 g_{3}^{2}+g_{3}^{\prime 2}\right) \lambda_{m, 3}-2 \lambda_{m, 3}^{2}-6 \lambda_{\sigma, 3}^{2}\right)\left(c+\ln \left(\frac{3 T}{\Lambda_{3 \mathrm{~d}}}\right)\right) \\
& +\frac{T^{2}}{(4 \pi)^{2}}\left[\frac{\left(2+3 L_{b}\right)}{24}\left(3 g^{2}+g^{\prime 2}\right) \lambda_{m}-L_{b}\left(\left(\lambda_{h}+\frac{7}{12} \lambda_{m}+\frac{1}{2} \lambda_{\sigma}\right) \lambda_{m}+\frac{9}{4} \lambda_{\sigma}^{2}\right)\right. \\
& \left.\quad-\frac{N_{\mathrm{c}}}{12}\left(3 L_{b}-L_{f}\right) g_{Y}^{2} \lambda_{m}\right] \\
& +\frac{\zeta_{3}}{(4 \pi)^{4}}\left(\left(\frac{1}{8}\left(3 g^{2}+g^{\prime 2}\right)+\frac{N_{\mathrm{c}}}{6} g_{Y}^{2}+\lambda_{h}+\frac{1}{12} \lambda_{m}\right) \mu_{m}^{2}+\left(\frac{4}{3} \lambda_{m}+2 \lambda_{\sigma}\right) \mu_{3}^{2}\right) \\
& -\frac{\zeta_{3}}{(4 \pi)^{6} T^{2}} \frac{\left(3+2 L_{b}\right)}{4}\left(32 \mu_{3}^{4}+8 \mu_{3}^{2} \mu_{m}^{2}+\mu_{m}^{4}\right),
\end{aligned}
$$

and

$$
\begin{aligned}
\mu_{1,3}=T^{-\frac{1}{2}}\left\{\mu_{1}(\Lambda)+\frac{T^{2}}{12}\left(\mu_{3}(\Lambda)+\mu_{m}(\Lambda)\right)-\frac{L_{b}}{(4 \pi)^{2}}\left(\mu_{m} \mu_{h}^{2}(\Lambda)+\mu_{3} \mu_{\sigma}^{2}(\Lambda)\right)\right. \\
\quad-\frac{\zeta_{3}}{(4 \pi)^{4} T^{2}} \frac{1}{6}\left(4 \mu_{3}^{2}+\mu_{m}^{2}\right)\left(\mu_{1}+\frac{1}{12} T^{2}\left(\mu_{3}+\mu_{m}\right)\right) \\
+\frac{1}{(4 \pi)^{4}} \frac{1}{8}\left(3+2 L_{b}+L_{b}^{2}\right)\left(8 \mu_{3}^{3}+2 \mu_{3} \mu_{m}^{2}+\mu_{m}^{3}\right) \\
+\frac{T^{2}}{(4 \pi)^{2}}\left[\frac{\left(2+3 L_{b}\right)}{48}\left(3 g^{2}+g^{\prime 2}\right) \mu_{m}-\frac{L_{b}}{2}\left(\left(\lambda_{h}+\frac{7}{12} \lambda_{m}\right) \mu_{m}+\left(\frac{1}{3} \lambda_{m}+\frac{3}{2} \lambda_{\sigma}\right) \mu_{3}\right)\right. \\
\left.\left.\quad-\frac{N_{\mathrm{c}}}{24} \mu_{m} g_{Y}^{2}\left(3 L_{b}-L_{f}\right)\right]\right\} \\
-\frac{1}{(4 \pi)^{2}}\left(2 \lambda_{\sigma, 3} \mu_{3,3}-\frac{1}{2} \mu_{m, 3}\left(3 g_{3}^{2}+g_{3}^{\prime 2}-2 \lambda_{m, 3}\right)\right)\left(c+\ln \left(\frac{3 T}{\Lambda_{3 \mathrm{~d}}}\right)\right) .
\end{aligned}
$$

We explicated the dependence of the $4 \mathrm{~d}$ RG-scale $\Lambda$ in terms where the running can be verified to cancel the logarithmic scale dependence, rendering the $3 \mathrm{~d}$ parameters $\Lambda$ independent. The running is determined by the $\beta$-functions listed in appendix A.1. In the $\mathrm{xSM}$ the cancellation of the RG-scale is slightly more subtle than in the SM, since the running of the masses starts at order $\mathcal{O}\left(\mu_{m}^{2}, \mu_{3}^{2}\right)$. Therefore, the running of the one-loop mass corrections is crucial. In the above formulas, the $3 \mathrm{~d}$ running is in fact not exact, as we did not include two-loop terms with singlet-temporal scalar couplings $x_{3}, x_{3}^{\prime}, y_{3}$ and $y_{3}^{\prime}$. However, this approximation is justified since temporal couplings only arise at $\mathcal{O}\left(g^{4}\right)$ because the singlet couples only indirectly to gauge fields. Hence, the omitted terms proportional to $x_{3}^{2}, \ldots$ are numerically negligible. The SM result for the three-dimensional 
mass parameter reads

$$
\begin{aligned}
&\left(\mu_{h, 3}^{2}\right)_{\mathrm{SM}}=\mu_{h}^{2}(\Lambda)+ \frac{T^{2}}{12}\left(\frac{3}{4}\left(3 g^{2}(\Lambda)+g^{\prime 2}(\Lambda)\right)+N_{\mathrm{c}} g_{Y}^{2}(\Lambda)+6 \lambda_{h}(\Lambda)\right) \\
&+ \frac{\mu_{h}^{2}(\Lambda)}{(4 \pi)^{2}}\left(\left(\frac{3}{4}\left(3 g^{2}+g^{\prime 2}\right)-6 \lambda_{h}\right) L_{b}-N_{\mathrm{c}} g_{Y}^{2} L_{f}\right) \\
&+\frac{T^{2}}{(4 \pi)^{2}}\left[\frac{167}{96} g^{4}+\frac{1}{288} g^{\prime 4}-\frac{3}{16} g^{2} g^{\prime 2}+\frac{\left(1+3 L_{b}\right)}{4} \lambda_{h}\left(3 g^{2}+g^{\prime 2}\right)\right. \\
&+L_{b}\left(\frac{17}{16} g^{4}-\frac{5}{48} g^{\prime 4}-\frac{3}{16} g^{2} g^{\prime 2}-6 \lambda_{h}^{2}\right) \\
&+\frac{1}{T^{2}}\left(c+\ln \left(\frac{3 T}{\Lambda_{3 \mathrm{~d}}}\right)\right)\left(\frac{39}{16} g_{3}^{4}+12 g_{3}^{2} h_{3}-6 h_{3}^{2}+9 g_{3}^{2} \lambda_{2,3}-12 \lambda_{h, 3}^{2}\right. \\
&-\frac{1}{96}\left(9 L_{b}-3 L_{f}-2\right)\left(\left(N_{\mathrm{c}}+1\right) g^{4}+\frac{1}{6} g_{3}^{2} g_{3}^{\prime 2}-2 h_{3}^{\prime 2} g^{\prime 4}\right) n_{\mathrm{f}} \\
&+\frac{N_{\mathrm{c}}}{32}\left(7 L_{b}-L_{f}-2\right) g^{2} g_{Y}^{2}-\frac{N_{\mathrm{c}}}{4}\left(3 L_{b}+L_{f}\right) \lambda_{h} g_{Y}^{2} \\
&+\frac{N_{\mathrm{c}}}{96}\left(\left(9\left(L_{b}-L_{f}\right)+4\right) Y_{\phi}^{2}-2\left(L_{b}-4 L_{f}+3\right)\left(Y_{q}^{2}+Y_{u}^{2}\right)\right) g^{\prime 2} g_{Y}^{2} \\
&\left.-\frac{N_{\mathrm{c}} C_{\mathrm{F}}}{6}\left(L_{b}-4 L_{f}+3\right) g_{\mathrm{s}}^{2} g_{Y}^{2}+\frac{N_{\mathrm{c}}}{24}\left(3 L_{b}-2\left(N_{\mathrm{c}}-3\right) L_{f}\right) g_{Y}^{4}\right], \quad(4.43)
\end{aligned}
$$

where $C_{\mathrm{F}}=\frac{N_{\mathrm{c}}^{2}-1}{2 N_{\mathrm{c}}}=\frac{4}{3}$ is the fundamental quadratic Casimir of $\mathrm{SU}(3)$. In the xSM the running of $\mu_{h}^{2}(\Lambda)$ starts at $\mathcal{O}\left(\mu_{m}^{2}\right)$ which is apparent from the $\beta$-function (A.15). Therefore, also the one-loop mass correction in the second line of $\mu_{h, 3}^{2}$ is affected by it which is required for the cancellation of the RG-scale. This result can be found in refs. [111, 157] where the latter neglects the two-loop contributions involving $g^{\prime}$. The matching relations of the marginal operator coefficients are listed in appendix A.3. Additionally also the couplings between temporal scalars and the Higgs doublet receive singlet-induced corrections:

$$
\begin{aligned}
h_{3}=\frac{g^{2}(\Lambda) T}{4}\left[1+\frac{1}{(4 \pi)^{2}}\left(\left(\frac{43}{6} L_{b}+\frac{17}{2}-\frac{\left(N_{\mathrm{c}}+1\right) n_{\mathrm{f}}}{3}\left(L_{f}-1\right)\right) g^{2}+\frac{g^{\prime 2}}{2}-2 N_{\mathrm{c}} g_{Y}^{2}+12 \lambda_{h}\right)\right. \\
\left.\quad-\frac{\zeta_{3}}{(4 \pi)^{4} T^{2}} \frac{2}{3} \mu_{m}^{2}\right] \\
h_{3}^{\prime}=\frac{g^{\prime 2}(\Lambda) T}{4}\left[1+\frac{1}{(4 \pi)^{2}}\left(\frac{3 g^{2}}{2}-\frac{1}{6}\left(\left(L_{b}-1\right) Y_{\phi}^{2}+\left(L_{f}-1\right) Y_{2 \mathrm{f}} n_{\mathrm{f}}\right) g^{\prime 2}\right.\right. \\
\left.\left.\quad-2\left(Y_{q}^{2}+Y_{u}^{2}\right) N_{\mathrm{c}} g_{Y}^{2}+12 \lambda_{h}\right)-\frac{\zeta_{3}}{(4 \pi)^{4} T^{2}} \frac{2}{3} \mu_{m}^{2}\right] \\
h_{3}^{\prime \prime}=\frac{g(\Lambda) g^{\prime}(\Lambda) T}{2}\left[1+\frac{1}{(4 \pi)^{2}}\left(\left(\frac{43}{12} L_{b}-1\right) g^{2}-\frac{Y_{\phi}^{2}}{3}\left(\frac{1}{4} L_{b}-1\right) g^{\prime 2}+4 \lambda_{h}+\frac{2}{3} N_{\mathrm{c}} g_{Y}^{2}\right.\right. \\
\left.\left.\quad-\left(L_{f}-1\right)\left(\frac{N_{\mathrm{c}}+1}{6} g^{2}+\frac{Y_{2 \mathrm{f}}}{12} g^{\prime 2}\right) n_{\mathrm{f}}\right)-\frac{\zeta_{3}}{(4 \pi)^{4} T^{2}} \frac{2}{3} \mu_{m}^{2}\right] .
\end{aligned}
$$


Due to the absence of singlet contributions, other 3d parameters in the dimensionally reduced Lagrangian (4.8) agree with the Standard model. For completeness, we collect these results below, with $n_{\mathrm{f}}=3$ the number of fermion generations

$$
\begin{aligned}
g_{3}^{2} & =g^{2}(\Lambda) T\left[1+\frac{g^{2}}{(4 \pi)^{2}}\left(\frac{43}{6} L_{b}+\frac{2}{3}-\frac{\left(N_{\mathrm{c}}+1\right) n_{\mathrm{f}}}{3} L_{f}\right)\right] \\
g_{3}^{\prime 2} & =g^{\prime 2}(\Lambda) T\left[1-\frac{g^{\prime 2}}{(4 \pi)^{2}} \frac{1}{6}\left(L_{b} Y_{\phi}^{2}+L_{f} Y_{2 \mathrm{f}} n_{\mathrm{f}}\right)\right] \\
m_{\mathrm{D}}^{2} & =g^{2} T^{2} \frac{1}{3}\left(\frac{5}{2}+\frac{\left(N_{\mathrm{c}}+1\right)}{4} n_{\mathrm{f}}\right) \\
m_{\mathrm{D}}^{\prime 2} & =g^{\prime 2} T^{2} \frac{1}{24}\left(4 Y_{\phi}^{2}+Y_{2 \mathrm{f}} n_{\mathrm{f}}\right) \\
m_{\mathrm{D}}^{\prime \prime 2} & =g_{\mathrm{s}}^{2} T^{2}\left(\frac{N_{\mathrm{c}}}{3}+\frac{n_{\mathrm{f}}}{3}\right) \\
\kappa_{3} & =T \frac{g^{4}}{(4 \pi)^{2}} \frac{1}{3}\left(17-\left(N_{\mathrm{c}}+1\right) n_{\mathrm{f}}\right) \\
\kappa_{3}^{\prime} & =T \frac{g^{\prime 4}}{(4 \pi)^{2}} \frac{1}{6}\left(2 Y_{\phi}^{4}-Y_{4 \mathrm{f}} n_{\mathrm{f}}\right) \\
\kappa_{3}^{\prime \prime} & =T \frac{g^{2} g^{\prime 2}}{(4 \pi)^{2}} 2\left(Y_{\phi}^{2}-\left(Y_{\ell}^{2}+N_{\mathrm{c}} Y_{q}^{2}\right) n_{\mathrm{f}}\right) .
\end{aligned}
$$

We point out, that eq. (3.87) in ref. [151] misprints the gluon Debye mass $m_{\mathrm{D}}^{\prime \prime 2}$, which we corrected above in eq. (4.51).

Two-loop electroweak Debye masses $\boldsymbol{m}_{\mathrm{D}}$ and $\boldsymbol{m}_{\mathrm{D}}^{\prime}$. Above, we quoted the one-loop electroweak Debye masses $m_{\mathrm{D}}$ and $m_{\mathrm{D}}^{\prime}$, which are standard, as their two-loop corrections are of higher order at final ultrasoft scale EFT. To reach a consistent $\mathcal{O}\left(g^{4}\right)$ order at the soft scale, these Debye masses should be computed at two-loop order, and at this order they receive contributions from the singlet. We point out, that the QCD Debye mass is independent of singlet contributions at two-loop order. We omit its two-loop result here, since these are further suppressed at the ultrasoft scale than two-loop contributions to the EW Debye masses because the Higgs couples only indirectly to the gluon sector.

The singlet-induced two-loop corrections to the electroweak Debye masses read

$$
\begin{aligned}
& m_{\mathrm{D}}^{\prime 2}=\frac{T^{2}}{(4 \pi)^{2}} \frac{1}{12} g^{\prime 2} \lambda_{m}-\frac{1}{(4 \pi)^{4}} g^{\prime 2} \mu_{m}^{2}\left(1+\frac{1}{2} L_{b}\right)+(\mathrm{SM} \text { terms }), \\
& m_{\mathrm{D}}^{2}=\frac{T^{2}}{(4 \pi)^{2}} \frac{1}{12} g^{2} \lambda_{m}-\frac{1}{(4 \pi)^{4}} g^{2} \mu_{m}^{2}\left(1+\frac{1}{2} L_{b}\right)+(\mathrm{SM} \text { terms }) .
\end{aligned}
$$

Their standard Model contributions have initially been computed in refs. [129, 130] and are reproduced in eqs. (A.112) and (A.113) in appendix A.4.

\subsection{Integrating out the soft scale}

The second step of dimensional reduction integrates out heavy temporal scalars at the soft scale. The resulting simplified 3d EFT at the ultrasoft scale assumes light, dynamical 
doublet and singlet scalars. Since the singlet couples to gauge fields only indirectly, its couplings to temporal scalars $A_{0}^{a}$ and $B_{0}$ are suppressed already at leading order. Hence, we include only one-loop effects of temporal scalars in ultrasoft singlet parameters instead of including two-loop corrections for tadpole and singlet mass parameter. All correlators are then encoded in the one-loop contribution of the effective potential (see appendix B for the one-loop master integral)

$$
V_{\mathrm{eff}, \text { soft }}^{11 \text { oop }} \simeq 3 J_{\mathrm{soft}}\left(m_{A}\right)+J_{\mathrm{soft}}\left(m_{B}\right)
$$

Denoting the background fields $v_{3}$ for the doublet and $s_{3}$ for the singlet, the (3d) background field-dependent mass eigenvalues read

$$
\begin{aligned}
& m_{A}^{2}=m_{\mathrm{D}}^{2}+h_{3} v_{3}^{2}+2 s_{3}\left(x_{3}+y_{3} s_{3}\right), \\
& m_{B}^{2}=m_{\mathrm{D}}^{\prime 2}+h_{3}^{\prime} v_{3}^{2}+2 s_{3}\left(x_{3}^{\prime}+y_{3}^{\prime} s_{3}\right),
\end{aligned}
$$

and give rise to the (one-loop) matching relations

$$
\begin{aligned}
& \bar{\mu}_{1,3}=\mu_{1,3}-\frac{1}{4 \pi}\left(3 m_{\mathrm{D}} x_{3}+m_{\mathrm{D}}^{\prime} x_{3}^{\prime}\right) \\
& \bar{\mu}_{\sigma, 3}^{2}=\mu_{\sigma, 3}^{2}-\frac{1}{2 \pi}\left(3 m_{\mathrm{D}} y_{3}+m_{\mathrm{D}}^{\prime} y_{3}^{\prime}\right)-\frac{1}{4 \pi}\left(3 \frac{x_{3}^{2}}{m_{\mathrm{D}}}+\frac{x_{3}^{\prime 2}}{m_{\mathrm{D}}^{\prime}}\right) \\
& \bar{\mu}_{3,3}=\mu_{3,3}-\frac{3}{4 \pi}\left(3 \frac{x_{3} y_{3}}{m_{\mathrm{D}}}+\frac{x_{3}^{\prime} y_{3}^{\prime}}{m_{\mathrm{D}}^{\prime}}\right)+\frac{1}{8 \pi}\left(3 \frac{x_{3}^{3}}{m_{\mathrm{D}}^{3}}+\frac{x_{3}^{\prime 3}}{m_{\mathrm{D}}^{\prime 3}}\right) \\
& \bar{\lambda}_{\sigma, 3}=\lambda_{\sigma, 3}-\frac{1}{8 \pi}\left(3 \frac{x_{3}^{4}}{m_{\mathrm{D}}^{5}}+\frac{x_{3}^{\prime 4}}{m_{\mathrm{D}}^{\prime 5}}\right)+\frac{1}{2 \pi}\left(3 \frac{x_{3}^{2} y_{3}}{m_{\mathrm{D}}^{3}}+\frac{x_{3}^{\prime 2} y_{3}^{\prime}}{m_{\mathrm{D}}^{\prime 3}}\right)-\frac{1}{2 \pi}\left(3 \frac{y_{3}^{2}}{m_{\mathrm{D}}}+\frac{y_{3}^{\prime 2}}{m_{\mathrm{D}}^{\prime}}\right), \\
& \bar{\mu}_{m, 3}=\mu_{m, 3}-\frac{1}{2 \pi}\left(3 \frac{h_{3} x_{3}}{m_{\mathrm{D}}}+\frac{h_{3}^{\prime} x_{3}^{\prime}}{m_{\mathrm{D}}^{\prime}}\right) \\
& \bar{\lambda}_{m, 3}=\lambda_{m, 3}+\frac{1}{4 \pi}\left(3 \frac{h_{3} x_{3}^{2}}{m_{\mathrm{D}}^{3}}+\frac{h_{3}^{\prime} x_{3}^{\prime 2}}{m_{\mathrm{D}}^{\prime 3}}\right)-\frac{1}{2 \pi}\left(3 \frac{h_{3} y_{3}}{m_{\mathrm{D}}}+\frac{h_{3}^{\prime} y_{3}^{\prime}}{m_{\mathrm{D}}^{\prime}}\right) .
\end{aligned}
$$

Therein soft corrections stem only from correlators since field normalisations contribute at a higher order due to non-existing tree-level contributions. In particular, all soft contributions at leading order are gauge-independent, as there are no gauge field propagators involved 
at one-loop order. The remaining parameters are referred from [111, 159, 172]

$$
\begin{aligned}
\bar{g}_{3}^{2}= & g_{3}^{2}\left(1-\frac{g_{3}^{2}}{6(4 \pi) m_{\mathrm{D}}}\right) \\
\bar{g}_{3}^{\prime 2}= & g_{3}^{\prime 2}, \\
\bar{\mu}_{h, 3}^{2}= & \mu_{h, 3}^{2}-\frac{1}{4 \pi}\left(3 h_{3} m_{\mathrm{D}}+h_{3}^{\prime} m_{\mathrm{D}}^{\prime}+2 N_{\mathrm{c}} C_{\mathrm{F}} \delta_{3} m_{\mathrm{D}}^{\prime \prime}\right) \\
& +\frac{1}{(4 \pi)^{2}}\left(3 g_{3}^{2} h_{3}-3 h_{3}^{2}-{h_{3}^{\prime}}^{2}-\frac{3}{2} h_{3}^{\prime \prime 2}\right. \\
& -\left(\frac{3}{4} g_{3}^{4}-12 g_{3}^{2} h_{3}\right) \ln \left(\frac{\Lambda_{3 \mathrm{~d}}}{2 m_{\mathrm{D}}}\right)-6 h_{3}^{2} \ln \left(\frac{\Lambda_{3 \mathrm{~d}}}{2 m_{\mathrm{D}}}\right) \\
& \left.-2{h_{3}^{\prime}}^{2} \ln \left(\frac{\Lambda_{3 \mathrm{~d}}}{2 m_{\mathrm{D}}^{\prime}}\right)-3 h_{3}^{\prime \prime 2} \ln \left(\frac{\Lambda_{3 \mathrm{~d}}}{m_{\mathrm{D}}+m_{\mathrm{D}}^{\prime}}\right)\right), \\
\bar{\lambda}_{3}= & \lambda_{3}-\frac{1}{2(4 \pi)}\left(\frac{3 h_{3}^{2}}{m_{\mathrm{D}}}+\frac{h_{3}^{\prime 2}}{m_{\mathrm{D}}^{\prime}}+\frac{h_{3}^{\prime \prime 2}}{m_{\mathrm{D}}+m_{\mathrm{D}}^{\prime}}\right) .
\end{aligned}
$$

In general the ultrasoft Higgs self-energy $\bar{\mu}_{h, 3}^{2}$ receives contributions from interactions with singlet and temporal scalars. Even though these are two-loop topologies, we discard them due to the suppression of $x_{3}, x_{3}^{\prime}, y_{3}, y_{3}^{\prime}$ in analogy with discarding contributions with quartic self-interactions of temporal scalars. This is apparent for $\kappa_{3}, \kappa_{3}^{\prime}, \kappa_{3}^{\prime \prime}$ that lack a tree-level contribution $\mathcal{O}\left(g^{2}\right)$ and consequently their leading contribution is $\mathcal{O}\left(g^{4}\right)$. For simplicity, we drop corrections from temporal scalars to marginal operators due to their numerical insignificance.

These relations complete our construction of the high- $T$ 3d EFT of the SM augmented with a real scalar singlet. As an effective theory, it can be used to examine the thermodynamics of the electroweak phase transition of the fundamental model (cf. refs. [172, 173]). In particular, ref. [172] showcases the computation of the two-loop thermal effective potential in the $3 \mathrm{~d}$ EFT of the xSM constructed in this section. This is analogous to our section 3.4.

\section{Discussion}

The pipeline between collider phenomenology of BSM theories and their implications to early universe cosmology and the potential birth of stochastic GW background convolves multiple complicated stages. One goal of this article is to take steps towards that, on theoretical grounds, uncertainties related to the prediction of the thermodynamics are not the largest in this pipeline.

Concretely, we gave a fresh qualitative review of the thermodynamics of the electroweak phase transition and focused on scalar extensions of the SM. In particular, we concentrated on the framework of high-temperature dimensional reduction. As an automatic all-order resummation scheme it perturbatively defers the infrared problem of thermal field theory. Thereafter, the IR sensitive physics is encoded in a dimensionally reduced EFT that can be studied non-perturbatively on the lattice. However, the constructed 3d EFT is powerful already in perturbative studies. The tutorial-styled computations explicated in section 3 aim 
to make this technique more accessible with emphasis on a scientific community studying the thermodynamics of the EWPT for a wide variety of BSM theories.

The majority of studies of the electroweak phase transition in BSM setups are restricted to perturbative computations of the thermal effective potential. They are often limited to one-loop order and naive leading order resummation usually of Arnold-Espinosa type. Therefrom, a straightforward extension to a non-perturbative treatment is less apparent and the IR problem remains in its core. On perturbative grounds, important contributions in the weak coupling expansion are missed which roots in a misalignment of loop and coupling (power counting) expansions. This omission causes a residual, artificial RG scale dependence which cannot be compensated by RG-improvement at one-loop. A recent study [159] concludes that this kind of leftover artificial renormalisation scale dependence can pose a dramatic two to three orders-of-magnitude theoretical uncertainty for subsequent analyses of the cosmic gravitational wave background originating from cosmic phase transitions. Such an uncertainty for thermodynamic parameters can compromise predictions for e.g. the signal-to-noise ratio for LISA and other future GW experiments.

Tools to automate dimensional reduction are much needed to handle large numbers of Feynman diagrams that arise at multi-loop orders. By adopting sophisticated tool from zero temperature, developments towards such automation have been taken recently [159, 171]. As a concrete application of dimensional reduction, we derived for the first time the high-temperature 3d EFT of the real-singlet extended Standard Model (with a dynamical singlet) - one of the most widely studied BSM models in particle cosmology. This poses the main technical part of our investigation displayed in section 4. Perturbative studies of this 3d EFT to scrutinise the EWPT in this model are presented in refs. [172, 173].

We conclude by envisioning specific but also model-independent future avenues:

(i) The derived 3d EFT of the xSM is indispensable for subsequent studies. Lattice simulations can probe its equilibrium thermodynamics and in particular expose the character of the phase transition and determine parameter regions that admit SFOPT. Additionally, out-of-equilibrium properties of the phase transition such as bubble nucleation rate can be investigated by non-perturbative studies of the $3 \mathrm{~d}$ EFT.

A leftover gauge dependence indicates an incomplete basis of higher dimensional operators in the dimensionally reduced theory. In general, it is interesting how their effects influence the IR dynamics of the system [225].

(ii) The real singlet scalar model (not coupled to SM) offers a testing platform for different approaches. Implications could be drawn for dark sector phase transitions, by determining the mapping of $4 \mathrm{~d}$ parameters and temperature to its $3 \mathrm{~d}$ phase structure. The latter was comprehensively analysed in ref. [160].

Since a real scalar theory is purely bosonic, it evades problems of discretising chiral fermions on lattice. Hence, a comparison between 3d EFT and full 4d lattice simulations is feasible (see related ref. [150]) also when including higher dimensional operators. In this context, its lattice-continuum relations with higher dimensional operators are needed. It remains to be determined which lattice measurements and 
extrapolations are required to extract the continuum physics from simulations in presence of higher dimensional operators.

(iii) It would be worth investigating how large parameter space scans of past EWPT studies (using a one-loop thermal potential) are affected when complete $\mathcal{O}\left(g^{4}\right)$ effects are included. We advocate pre-existing software to implement the perturbative dimensionally reduced 3d EFT approach. An example are parameter space scans using CosmoTransitions [215], BSMPT [214], PhaseTracer [216] to examine the phase structure of individual BSM models.

\section{Acknowledgments}

Over the years of this work, the authors would like to thank Jens O. Andersen, Tomáš Brauner, Djuna Croon, Daniel Cutting, Ioan Ghişoiu, Tyler Gorda, Oliver Gould, Mark Hindmarsh, Keijo Kajantie, Thomas Konstandin, Jonathan Kozaczuk, Mikko Laine, Lauri Niemi, Jose Miguel No, Hiren Patel, Michael J. Ramsey-Musolf, Arttu Rajantie, Kari Rummukainen, York Schröder, Anders Tranberg, Aleksi Vuorinen, Jorinde van de Vis, David J. Weir and Graham White for enlightening discussions. TT thanks Sara Tähtinen for crosschecking many individual Feynman diagrams, and correcting multiple related symmetry and combinatorial factors. This work was partly supported by the Swiss National Science Foundation (SNF) under grant 200020B-188712. PS has been supported by the European Research Council, grant no. 725369, and by the Academy of Finland, grant no. 1322507. JÖ acknowledges financial support from the Vilho, Yrjö and Kalle Väisälä Foundation of the Finnish Academy of Science and Letters.

\section{A Detailed computation in the $\mathrm{xSM}$}

This appendix collects details of the dimensional reduction computation in the xSM from section 4 and extends our results of the $3 \mathrm{~d}$ parameters to marginal operators defined in eq. (4.7).

\section{A.1 Counterterms and $\beta$-functions of the $4 d$ theory}

One-loop counterterms and $\beta$-functions are listed e.g. in section 3.2 in ref. [151]. Using field renormalisations $Z_{\phi}$ for the Higgs, $Z_{q}$ the left handed quark doublet and $Z_{t}$ the top quark, we define the bare top Yukawa parameter

$$
g_{Y(b)} \equiv Z_{\phi}^{-\frac{1}{2}} Z_{q}^{-\frac{1}{2}} Z_{t}^{-\frac{1}{2}} \Lambda^{\epsilon}\left(g_{Y}+\delta g_{Y}\right) .
$$

This convention for $g_{Y}$ and its counterterm $\delta g_{Y}$ align with eqs. (C.22) and (C.29) in ref. [157] in contrast to eqs. (2.20) and (3.38) of ref. [151]. Since these references use Landau gauge, 
we merely display the $\xi$-dependent counterterms in general covariant gauge:

$$
\begin{aligned}
\delta Z_{\phi} & =\frac{1}{(4 \pi)^{2}} \frac{1}{\epsilon}\left(\frac{3}{4}\left(3-\xi_{2}\right) g^{2}+\frac{1}{4}\left(3-\xi_{1}\right) g^{\prime 2}-N_{\mathrm{c}} g_{Y}^{2}\right) \\
\delta Z_{A} & =\frac{1}{(4 \pi)^{2}} \frac{1}{\epsilon} g^{2}\left(\frac{25}{6}-\frac{1}{3} n_{\mathrm{f}}\left(1+N_{\mathrm{c}}\right)-\xi_{2}\right) \\
\delta Z_{B} & =-\frac{1}{(4 \pi)^{2}} \frac{1}{\epsilon} g^{\prime 2} \frac{1}{6}\left(Y_{\phi}^{2}+Y_{2 \mathrm{f}} n_{\mathrm{f}}\right) \\
\delta Z_{q} & =-\frac{1}{(4 \pi)^{2}} \frac{1}{\epsilon}\left(\frac{1}{2} g_{Y}^{2}+\frac{3}{4} g^{2} \xi_{2}+\frac{1}{4} g^{\prime 2} Y_{q}^{2} \xi_{1}+C_{\mathrm{F}} g_{\mathrm{s}}^{2} \xi_{3}\right), \\
\delta Z_{u} & =-\frac{1}{(4 \pi)^{2}} \frac{1}{\epsilon}\left(g_{Y}^{2}+\frac{1}{4} g^{\prime 2} Y_{u}^{2} \xi_{1}+C_{\mathrm{F}} g_{\mathrm{s}}^{2} \xi_{3}\right), \\
\delta g_{Y} & =-\frac{1}{(4 \pi)^{2}} \frac{1}{\epsilon}\left(\frac{3 Y_{q} Y_{u}}{4} g^{\prime 2}+\frac{3}{4} g^{2} \xi_{2}+\frac{Y_{q} Y_{u}-Y_{\phi}\left(Y_{q}-Y_{u}\right)}{4} g^{\prime 2} \xi_{1}+C_{\mathrm{F}} g_{\mathrm{s}}^{2}\left(3+\xi_{3}\right)\right) \\
\delta \lambda_{h} & =\frac{1}{(4 \pi)^{2}} \frac{1}{\epsilon}\left(\frac{3}{16}\left(3 g^{4}+2 g^{2} g^{\prime 2}+g^{\prime 4}\right)-3 g_{Y}^{4}+12 \lambda_{h}+\frac{1}{4} \lambda_{m}-\frac{1}{2} \lambda_{h}\left(3 g^{2} \xi_{2}+g^{\prime 2} \xi_{1}\right)\right), \\
\delta \mu_{m} & =\frac{1}{(4 \pi)^{2}} \frac{1}{\epsilon}\left(6 \lambda_{h} \mu_{m}+2 \lambda_{m}\left(\mu_{m}+\mu_{3}\right)-\frac{1}{4} \mu_{m}\left(3 g^{2} \xi_{2}+g^{\prime 2} \xi_{1}\right)\right), \\
\delta \lambda_{m} & =\frac{1}{(4 \pi)^{2}} \frac{1}{\epsilon} \lambda_{m}\left(6 \lambda_{h}+2 \lambda_{m}+3 \lambda_{\sigma}-\frac{1}{4}\left(3 g^{2} \xi_{2}+g^{\prime 2} \xi_{1}\right)\right),
\end{aligned}
$$

where hypercharges are defined in eq. (4.16). Essentially also the unphysical gauge fixing parameter receives renormalisation: $\xi_{(b)}=\xi\left(1+\delta Z_{\xi}\right)$ with $\delta Z_{\xi_{1}}=\delta Z_{B}$ and $\delta Z_{\xi_{2}}=\delta Z_{A}$.

The two-loop computation of tadpole and mass parameters receives contributions that require new counterterms:

$$
\begin{aligned}
\delta \mu_{1}= & \frac{1}{(4 \pi)^{2}} \frac{1}{\epsilon}\left(\mu_{h}^{2} \mu_{m}+\mu_{\sigma}^{2} \mu_{3}\right)+\frac{1}{(4 \pi)^{4}}\left(\frac{1}{\epsilon^{2}}-\frac{1}{\epsilon}\right)\left(\frac{1}{8} \mu_{m}^{3}+\mu_{3}^{3}+\frac{1}{4} \mu_{3} \mu_{m}^{2}\right), \\
\delta \mu_{h}^{2}= & \frac{1}{(4 \pi)^{2}} \frac{1}{\epsilon} \frac{1}{4}\left(24 \lambda_{h} \mu_{h}^{2}+\mu_{m}^{2}+2 \lambda_{m} \mu_{\sigma}^{2}-\mu_{h}^{2}\left(g^{\prime 2} \xi_{1}+3 g^{2} \xi_{2}\right)\right) \\
& -\frac{1}{(4 \pi)^{4}} \frac{1}{\epsilon}\left(\frac{1}{2} \lambda_{m}\left(\mu_{3}^{2}+\mu_{3} \mu_{m}\right)-\mu_{m}^{2}\left(\frac{1}{32}\left(3 g^{2}+g^{\prime 2}\right)-\frac{N_{\mathrm{c}}}{8} g_{Y}^{2}-\frac{9}{4} \lambda_{h}-\frac{3}{8} \lambda_{m}\right)\right) \\
& +\frac{1}{(4 \pi)^{4}} \frac{1}{\epsilon^{2}}\left(\frac{1}{2} \lambda_{m}\left(\mu_{3}^{2}+\mu_{3} \mu_{m}\right)\right. \\
\delta \mu_{\sigma}^{2}= & \frac{1}{(4 \pi)^{2}} \frac{1}{\epsilon} \frac{1}{2}\left(6 \lambda_{\sigma} \mu_{\sigma}^{2}+4 \lambda_{m} \mu_{h}^{2}+4 \mu_{3}^{2}+\mu_{m}^{2}\right) \\
& -\frac{1}{(4 \pi)^{4}} \frac{1}{\epsilon}\left(15 \lambda_{\sigma} \mu_{3}^{2}+2 \lambda_{m} \mu_{3} \mu_{m}-\mu_{m}^{2}\left(\frac{1}{2}\left(3 g^{2}+g^{\prime 2}\right)-\frac{N_{\mathrm{c}}}{2} g_{Y}^{2}-\frac{3}{4} \lambda_{\sigma}-\frac{5}{4} \lambda_{m}\right)\right) \\
& +\frac{1}{(4 \pi)^{4}} \frac{1}{\epsilon^{2}}\left(21 \lambda_{\sigma} \mu_{3}^{2}+4 \lambda_{m} \mu_{3} \mu_{m}-\mu_{m}^{2}\left(\frac{3}{8}\left(3 g^{2}+g^{\prime 2}\right)-\frac{N_{\mathrm{c}}}{2} g_{Y}^{2}-\frac{3}{4} \lambda_{\sigma}-\frac{5}{4} \lambda_{m}-3 \lambda_{h}\right)\right),
\end{aligned}
$$


and their corresponding $\beta$-functions are

$$
\begin{aligned}
\Lambda \frac{\mathrm{d}}{\mathrm{d} \Lambda} \mu_{1}= & \frac{1}{(4 \pi)^{2}} 2\left(\mu_{h}^{2} \mu_{m}+\mu_{\sigma}^{2} \mu_{3}\right)-\frac{1}{(4 \pi)^{4}}\left(\frac{1}{2} \mu_{m}^{3}+4 \mu_{3}^{3}+\mu_{3} \mu_{m}^{2}\right) \\
\Lambda \frac{\mathrm{d}}{\mathrm{d} \Lambda} \mu_{h}^{2}= & \frac{1}{(4 \pi)^{2}} 2\left(\frac{1}{4} \mu_{m}^{2}+\frac{1}{2} \lambda_{m} \mu_{\sigma}^{2}-\mu_{h}^{2}\left(\frac{3}{4}\left(3 g^{2}+g^{\prime 2}\right)-N_{\mathrm{c}} g_{Y}^{2}-6 \lambda_{h}\right)\right) \\
& -\frac{1}{(4 \pi)^{4}}\left(2 \lambda_{m} \mu_{3}^{2}+2 \lambda_{m} \mu_{3} \mu_{m}-\mu_{m}^{2}\left(\frac{1}{8}\left(3 g^{2}+g^{\prime 2}\right)-\frac{N_{\mathrm{c}}}{2} g_{Y}^{2}-9 \lambda_{h}-\frac{3}{2} \lambda_{m}\right)\right), \\
\Lambda \frac{\mathrm{d}}{\mathrm{d} \Lambda} \mu_{\sigma}^{2}= & \frac{1}{(4 \pi)^{2}} 2\left(2 \mu_{3}^{2}+\frac{1}{2} \mu_{m}^{2}+3 \lambda_{\sigma} \mu_{\sigma}^{2}+2 \lambda_{m} \mu_{h}^{2}\right) \\
& -\frac{1}{(4 \pi)^{4}} 2\left(30 \lambda_{\sigma} \mu_{3}^{2}+4 \lambda_{m} \mu_{3} \mu_{m}-\mu_{m}^{2}\left(\left(3 g^{2}+g^{\prime 2}\right)-N_{\mathrm{c}} g_{Y}^{2}-\frac{3}{2} \lambda_{\sigma}-\frac{5}{2} \lambda_{m}\right)\right),
\end{aligned}
$$

which are necessarily gauge-independent. The remaining $\beta$-functions are listed in section 3.2 of ref. [151].

\section{A.2 Correlators from the one-loop effective potential in general covariant gauge}

In the background field method, the scalar fields are shifted by $\phi_{i} \rightarrow \phi_{i}+\delta_{i, 2} v / \sqrt{2}$ for $i=1,2$ and $\sigma \rightarrow \sigma+s$, around real background fields $v$ and $s$. We can read the scalar correlators from the effective potential expanded in these background fields

$$
\begin{aligned}
V_{\mathrm{eff}}= & V_{0,0}+\frac{1}{2} V_{2,0} v^{2}+\frac{1}{4} V_{4,0} v^{4}+V_{0,1} s+V_{0,2} s^{2} \\
& +V_{0,3} s^{3}+V_{0,4} s^{4}+\frac{1}{2} V_{2,1} v^{2} s+\frac{1}{2} V_{2,2} v^{2} s^{2} \\
& +V_{0,5} s^{5}+\frac{1}{2} V_{2,3} v^{2} s^{3}+\frac{1}{4} V_{4,1} v^{4} s \\
& +\frac{1}{8} V_{6,0} v^{3}+V_{0,6} s^{6}+\frac{1}{4} V_{4,2} v^{4} s^{2}+\frac{1}{2} V_{2,4} v^{2} s^{4},
\end{aligned}
$$

up to dimension- 6 terms. In our convention, the coefficients $V$ relate to correlators appearing in the matching relations of eqs. (4.19)-(4.30) as

$$
\Gamma_{\left(\phi^{\dagger} \phi\right)^{2}}=8 V_{4,0}, \quad \Gamma_{\sigma^{4}}=24 V_{0,4}, \quad \Gamma_{\phi^{\dagger} \phi \sigma^{2}}=4 V_{2,2}, \quad \Pi_{\phi^{\dagger} \phi \sigma}=2 V_{2,1}, \quad \Pi_{\sigma^{3}}=6 V_{4,0},
$$

and similarly for 1- and 2-point correlators and marginal operators.

In Landau gauge, the background-dependent mass eigenvalues can be solved from the mass matrix constructed from the coefficients of the bilinear parts of the $\phi_{i^{-}}$and $\sigma$-fields that mix in the broken phase. By employing the shorthand notation for the parameters of the shifted theory

$$
\begin{aligned}
\tilde{\mu}_{h}^{2} & \equiv \mu_{h}^{2}+\lambda_{h} v^{2}+\frac{1}{2} \mu_{m} s+\frac{1}{2} \lambda_{m} s^{2}, \\
\tilde{\mu}_{\sigma}^{2} & \equiv \mu_{\sigma}^{2}+2 \mu_{3} s+3 \lambda_{\sigma} s^{2}+\frac{1}{2} \lambda_{m} v^{2}, \\
\tilde{\mu}_{3} & \equiv \mu_{3}+3 \lambda_{\sigma} s, \\
\tilde{\mu}_{m} & \equiv \mu_{m}+2 \lambda_{m} s,
\end{aligned}
$$


the scalar mass eigenvalues read

$$
\begin{aligned}
& m_{G}^{2}=\tilde{\mu}_{h}^{2}, \\
& m_{ \pm}^{2}=\frac{1}{2}\left(\tilde{\mu}_{h}^{2}+\tilde{\mu}_{\sigma}^{2}\right)+\lambda_{h} v^{2} \pm \sqrt{\left(\frac{1}{2}\left(-\tilde{\mu}_{h}^{2}+\tilde{\mu}_{\sigma}^{2}\right)-\lambda_{h} v^{2}\right)^{2}+v^{2}\left(\frac{1}{2} \mu_{m}+\lambda_{m} s\right)^{2}},
\end{aligned}
$$

where the Goldstone mass eigenvalue $m_{G}^{2}$ is triple degenerate. Since the singlet does not couple to the gauge fields or top quark, their mass eigenvalues align with the SM

$$
\begin{aligned}
m_{W^{ \pm}}^{2} & =\frac{1}{4} g^{2} v^{2}, & m_{Z}^{2} & =\frac{1}{4}\left(g^{2}+g^{\prime 2}\right) v^{2}, \\
m_{\gamma}^{2} & =0, & m_{t}^{2} & =\frac{1}{2} g_{Y}^{2} v^{2} .
\end{aligned}
$$

In general covariant gauge, the three Goldstone mass eigenvalues are replaced by $[170,236]$

$$
\begin{aligned}
& {m_{1}^{ \pm 2}}^{2}=\frac{1}{2}\left(m_{G}^{2} \pm \sqrt{m_{G}^{2}\left(m_{G}^{2}-\xi_{2} g^{2} v^{2}-\xi_{1} g^{\prime 2} v^{2}\right)}\right) \\
& m_{2}^{ \pm 2}=\frac{1}{2}\left(m_{G}^{2} \pm \sqrt{m_{G}^{2}\left(m_{G}^{2}-\xi_{2} g^{2} v^{2}\right)}\right)
\end{aligned}
$$

where $m_{2}^{ \pm 2}$ is double degenerate. Based on these background field dependent mass eigenvalues, the one-loop effective potential becomes

$$
\begin{aligned}
V_{\text {eff }}^{\text {lloop }}= & J_{b}\left(m_{1}^{+}\right)+J_{b}\left(m_{1}^{-}\right)+2 J_{b}\left(m_{2}^{+}\right)+2 J_{b}\left(m_{2}^{-}\right)+J_{b}\left(m_{+}\right)+J_{b}\left(m_{-}\right) \\
& +d\left(2 J_{b}\left(m_{W}^{ \pm}\right)+J_{b}\left(m_{Z}\right)\right)-4 N_{\mathrm{c}} J_{f}\left(m_{t}\right) .
\end{aligned}
$$

And by comparing to the expansion (A.17), we can solve for the desired correlators. For a crosscheck, we also compute all one-loop correlators directly in the unbroken phase.

\section{A.3 Matching of marginal operators}

Marginal operators of eq. (4.7) arise at $\mathcal{O}\left(g^{5}\right)$ and $\mathcal{O}\left(g^{6}\right)$, at which contributions from the field normalisations are absent in their matching. We get

$$
\begin{aligned}
c_{6,0}=T^{2}\left[\frac { \zeta _ { 3 } } { ( 4 \pi ) ^ { 4 } T ^ { 2 } } \left(\frac{3}{8} g^{6}+\frac{3}{8} g^{4} g^{\prime 2}+\frac{3}{8} g^{2} g^{\prime 4}+\frac{1}{8} g^{\prime 6}-\frac{28}{3} N_{\mathrm{c}} g_{Y}^{6}+80 \lambda_{h}^{3}+\frac{1}{3} \lambda_{m}^{3}\right.\right. \\
\left.\quad-2 \lambda_{h}^{2}\left(3 g^{2} \xi_{2}+g^{\prime 2} \xi_{1}\right)\right) \\
\quad-\frac{\zeta_{5}}{(4 \pi)^{6} T^{4}} \mu_{m}^{2}\left(36 \lambda_{h}^{2}+6 \lambda_{h} \lambda_{m}+\lambda_{m}^{2}\right) \\
\left.\quad+\frac{\zeta_{7}}{(4 \pi)^{8} T^{6}} \frac{5}{4}\left(6 \lambda_{h}+\lambda_{m}\right) \mu_{m}^{4}-\frac{\zeta_{9}}{(4 \pi)^{10} T^{8}} \frac{7}{12} \mu_{m}^{6}\right], \\
c_{0,6}=T^{2}\left[\frac{\zeta_{3}}{(4 \pi)^{4} T^{2}}\left(\frac{1}{6} \lambda_{m}^{3}+9 \lambda_{\sigma}^{3}\right)-\frac{\zeta_{5}}{(4 \pi)^{6} T^{4}} \frac{3}{4}\left(144 \lambda_{\sigma}^{2} \mu_{3}^{2}+\lambda_{m}^{2} \mu_{m}^{2}\right)\right. \\
\left.\quad+\frac{\zeta_{7}}{(4 \pi)^{8} T^{6}}\left(240 \lambda_{\sigma} \mu_{3}^{4}+\frac{5}{8} \lambda_{m} \mu_{m}^{4}\right)-\frac{\zeta_{9}}{(4 \pi)^{10} T^{8}} \frac{7}{48}\left(1024 \mu_{3}^{6}+\mu_{m}^{6}\right)\right],
\end{aligned}
$$




$$
\begin{aligned}
& c_{4,2}=T^{2}\left[\frac{\zeta_{3}}{(4 \pi)^{4} T^{2}} \lambda_{m}\left(24 \lambda_{h}^{2}+2 \lambda_{m}^{2}+\lambda_{m}\left(12 \lambda_{h}+3 \lambda_{\sigma}\right)-\lambda_{h}\left(3 g^{2} \xi_{2}+g^{\prime 2} \xi_{1}\right)\right)\right. \\
& -\frac{\zeta_{5}}{(4 \pi)^{6} T^{4}}\left(6 \lambda_{m} \mu_{m}\left(8 \lambda_{h} \mu_{3}+5 \lambda_{h} \mu_{m}+\lambda_{\sigma} \mu_{m}\right)+\lambda_{m}^{2}\left(12 \mu_{3}^{2}+16 \mu_{3} \mu_{m}+\frac{17}{2} \mu_{m}^{2}\right)\right. \\
& \left.+\mu_{m}^{2}\left(36 \lambda_{h}^{2}+18 \lambda_{h} \lambda_{\sigma}-\frac{3}{2} \lambda_{h}\left(3 g^{2} \xi_{2}+g^{\prime 2} \xi_{1}\right)+\frac{1}{16}\left(3 g^{4} \xi_{2}^{2}+2 g^{2} g^{\prime 2} \xi_{2} \xi_{1}+g^{\prime 4} \xi_{1}^{2}\right)\right)\right) \\
& +\frac{\zeta_{7}}{(4 \pi)^{8} T^{6}} \frac{5}{4} \mu_{m}^{2}\left(24\left(2 \lambda_{h}+\lambda_{m}\right) \mu_{3}^{2}+4\left(6 \lambda_{h}+5 \lambda_{m}\right) \mu_{3} \mu_{m}+\left(9 \lambda_{h}+5 \lambda_{m}+3 \lambda_{\sigma}\right) \mu_{m}^{2}\right) \\
& \left.-\frac{\zeta_{9}}{(4 \pi)^{10} T^{8}} \frac{7}{16} \mu_{m}^{4}\left(48 \mu_{3}^{2}+16 \mu_{3} \mu_{3}+3 \mu_{m}^{2}\right)\right], \\
& c_{2,4}=\left[\frac{\zeta_{3}}{(4 \pi)^{4} T^{2}}\left(\lambda_{m}^{3}+9 \lambda_{m} \lambda_{\sigma}^{2}+3 \lambda_{m}^{2}\left(\lambda_{h}+2 \lambda_{\sigma}\right)-\frac{1}{8} \lambda_{m}^{2}\left(3 g^{2} \xi_{2}+g^{\prime 2} \xi_{1}\right)\right)\right. \\
& -\frac{\zeta_{5}}{(4 \pi)^{6} T^{4}}\left(9 \lambda_{\sigma}^{2} \mu_{m}^{2}+\frac{1}{4} \lambda_{m}^{2}\left(64 \mu_{3}^{2}+32 \mu_{3} \mu_{m}+13 \mu_{m}^{2}\right)\right. \\
& \left.+\frac{3}{8} \lambda_{m}\left(4 \lambda_{\sigma}\left(4 \mu_{3}+\mu_{m}\right)\left(12 \mu_{3}+5 \mu_{m}\right)+24 \mu_{m}^{2} \lambda_{h}\right)-\frac{3}{8} \lambda_{m}\left(3 g^{2} \xi_{2}+g^{\prime 2} \xi_{1}\right)\right) \\
& +\frac{\zeta_{7}}{(4 \pi)^{8} T^{6}} \frac{5}{32} \mu_{m}^{2}\left(12 \lambda_{\sigma} \mu_{m}^{2}\left(48 \mu_{3}^{3}+8 \mu_{3} \mu_{m}+\mu_{m}^{2}\right)\right. \\
& +2 \lambda_{m}\left(256 \mu_{3}^{4}+256 \mu_{3}^{3} \mu_{m}+80 \mu_{3}^{2} \mu_{m}^{2}+24 \mu_{3} \mu_{m}^{3}+7 \mu_{m}^{4}\right) \\
& \left.+24 \lambda_{h} \mu_{m}^{4}-\mu_{m}^{4}\left(g^{\prime 2} \xi_{1}+g^{2} \xi_{2}\right)\right) \\
& \left.-\frac{\zeta_{9}}{(4 \pi)^{10} T^{8}} \frac{7}{16} \mu_{m}^{2}\left(256 \mu_{3}^{4}+64 \mu_{3}^{3} \mu_{m}+16 \mu_{3}^{2} \mu_{m}^{2}+4 \mu_{3} \mu_{m}^{3}+\mu_{m}^{4}\right)\right], \\
& c_{0,5}=T^{\frac{3}{2}}\left[\frac{\zeta_{3}}{(4 \pi)^{4} T^{2}}\left(18 \lambda_{\sigma}^{2} \mu_{3}+\frac{1}{2} \lambda_{m}^{2} \mu_{m}\right)-\frac{\zeta_{5}}{(4 \pi)^{6} T^{4}}\left(48 \lambda_{\sigma} \mu_{3}^{3}+\frac{1}{2} \lambda_{m} \mu_{m}^{3}\right)\right. \\
& \left.+\frac{\zeta_{7}}{(4 \pi)^{8} T^{6}}\left(32 \mu_{3}^{5}+\frac{1}{8} \mu_{m}^{5}\right)\right] \\
& c_{2,3}=T^{\frac{3}{2}}\left[\frac{\zeta_{3}}{(4 \pi)^{4} T^{2}} \frac{\lambda_{m}}{4}\left(8\left(\lambda_{m}+3 \lambda_{\sigma}\right)\left(2 \mu_{3}+\mu_{m}\right)+24 \lambda_{h} \mu_{m}-\mu_{m}\left(3 g^{2} \xi_{2}+g^{\prime 2} \xi_{1}\right)\right)\right. \\
& -\frac{\zeta_{5}}{(4 \pi)^{6} T^{4}} \frac{1}{8}\left(4 \lambda_{m}\left(4 \mu_{3}+3 \mu_{m}\right)\left(8 \mu_{3}^{2}+2 \mu_{3} \mu_{m}+\mu_{m}^{2}\right)\right. \\
& \left.+12 \mu_{m}^{2}\left(\lambda_{\sigma}\left(8 \mu_{3}+\mu_{m}\right)+2 \mu_{m} \lambda_{h}\right)-\mu_{m}^{3}\left(3 g^{2} \xi_{2}+g^{\prime 2} \xi_{1}\right)\right) \\
& \left.+\frac{\zeta_{7}}{(4 \pi)^{8} T^{6}} \frac{5}{16} \mu_{m}^{2}\left(4 \mu_{3}+\mu_{m}\right)\left(16 \mu_{3}^{2}+\mu_{m}^{2}\right)\right], \\
& c_{4,1}=T^{\frac{3}{2}}\left[\frac{\zeta_{3}}{(4 \pi)^{4} T^{2}}\left(12 \lambda_{h} \lambda_{m} \mu_{m}+2 \lambda_{m}^{2}\left(\mu_{3}+\mu_{m}\right)+24 \lambda_{h}^{2} \mu_{m}-\lambda_{h} \mu_{m}\left(3 g^{2} \xi_{2}+g^{\prime 2} \xi_{1}\right)\right)\right. \\
& -\frac{\zeta_{5}}{(4 \pi)^{6} T^{4}} \frac{1}{2} \mu_{m}^{2}\left(12 \lambda_{h}\left(2 \mu_{3}+\mu_{m}\right)+\lambda_{m}\left(8 \mu_{3}+5 \mu_{m}\right)\right) \\
& \left.+\frac{\zeta_{7}}{(4 \pi)^{8} T^{6}} \frac{5}{8} \mu_{m}^{4}\left(4 \mu_{3}+\mu_{m}\right)\right] \text {. }
\end{aligned}
$$

Notably, all coefficients related to operators with Higgs field are gauge dependent, in analogy to the Higgs-singlet portal coupling in eq. (4.35). The class of topologies responsible for 
this uncancelled contribution can be exemplified by the Higgs-singlet portal interaction and sextic Higgs marginal operator

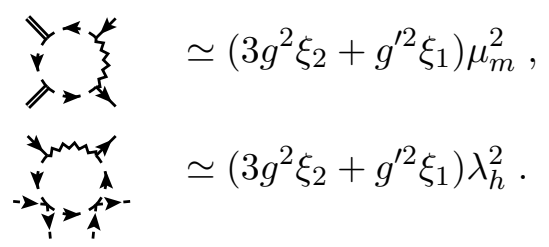

At zero external momenta the first diagram vanishes only in Landau gauge due its transversality, since it is proportional to $\mathscr{\&}_{P} P_{\mu} P_{\nu} D_{\mu \nu}(P) / P^{8}=\xi \mathscr{\&}_{P} 1 / P^{6}$. Where $D_{\mu \nu}(P)$ is the gauge field propagator in covariant gauge from eq. (A.38). Identically, the second diagram contributes to the Higgs 6-point correlator (A.29) where also a leftover gauge dependence remains. Similarly, other marginal operators with external Higgs legs are $\xi$-dependent. This leftover $\mathcal{O}\left(g^{6}\right)$ gauge dependence of the sextic correlator was pointed out in ref. [159]. Strikingly, the gauge dependence of the Higgs-singlet portal coupling $\lambda_{m}$ arises already at $\mathcal{O}\left(g^{4}\right)$. This underlines the subtlety of the power counting of the cubic portal coupling $\mu_{m}$ as discussed in section 4 .

Higher dimensional operators can be used to estimate the accuracy of the dimensional reduction by adding their effect at tree-level to $3 \mathrm{~d}$ effective potential. It remains to be understood how a gauge-invariant analysis is to be performed as some of these operators are explicitly gauge-dependent. We leave this endeavour as a future challenge, and note that at this stage these operators can be used as mere numerical estimates of the convergence of perturbation theory.

\section{A.4 Two-loop computation of correlators}

This appendix documents diagram-by-diagram the results for the two-loop correlators used in section 4.2 in terms of master sum-integrals of appendix B. Despite being an algorithmic loop-diagrammatic exercise, we believe that this explicit documentation can facilitate future endeavours of dimensionally reduced high- $T$ effective theories. Especially as one might find this to be the non-trivial part of a dimensional reduction computation. The computation was performed in general covariant gauge, where gauge parameters enter via gauge field propagators for $\mathrm{SU}(2)$

$$
\begin{aligned}
\left\langle A_{\mu}^{a}(P) A_{\nu}^{b}(-P)\right\rangle & =\delta^{a b} D_{\mu \nu}(P) \\
& =\frac{\delta^{a b}}{P^{2}}\left(\mathcal{P}_{\mu \nu}^{T}(P)+\xi \frac{P_{\mu} P_{\nu}}{P^{2}}\right)
\end{aligned}
$$

and similarly for other gauge propagators of $\mathrm{U}(1)$ and $\mathrm{SU}(3)$. The transverse projector is defined as $\mathcal{P}_{\mu \nu}^{T}(P) \equiv \delta_{\mu \nu}-P_{\mu} P_{\nu} / P^{2}$. This section displays results compactly employing Landau gauge $(\xi=0)$ where gauge propagators are transversal. This transversality decimates the number of integrals. For the Higgs self-energy, we only list new contributions of the singlet scalar.

The corresponding Feynman rules and conventions (in Landau gauge) are outlined in ref. [151]. The pure singlet diagrams are listed above in section 3.2.2, wherefore here we 
only include diagrams wherein the singlet couples to the SM. Note, that in this case also the pure singlet counterterm diagrams contains SM contributions, and similarly the Higgs counterterms have singlet contributions. Again the correlator is minus sum of Feynman diagrams, and at two-loop level (two-loop diagrams, one-loop counterterm diagrams) we employ massless propagators sufficient for a NLO dimensional reduction; see discussion at the end of section 3.2.2.

Singlet tadpole. Diagrammatically, the renormalised singlet tadpole correlator $\hat{\Gamma}_{\sigma}$ in Landau gauge composes of (excluding pure singlet terms eqs. (c.1)-(c.7))

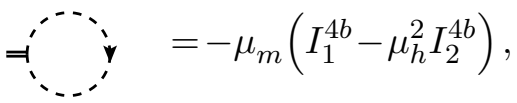

$$
\begin{aligned}
& =\text { ay }=6 \lambda_{h} \mu_{m} I_{2}^{4 b} I_{1}^{4 b} \\
& =\frac{1}{2} \lambda_{m} \mu_{m} I_{2}^{4 b} I_{1}^{4 b}, \\
& =0 . y=2 \lambda_{m} \mu_{3} I_{2}^{4 b} I_{1}^{4 b} \text {, }
\end{aligned}
$$

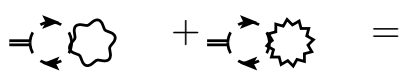

$$
\begin{aligned}
& +\frac{1}{4} \mu_{m}\left(3 g^{3}+g^{\prime 2}\right) d I_{2}^{4 b} I_{1}^{4 b}, \\
& =10=-2 \mu_{m} N_{\mathrm{c}} g_{Y}^{2} F_{5} \text {, } \\
& =\text { 디 } \\
& \stackrel{-\because}{\longleftarrow}=\mu_{m} \lambda_{m} S_{3}, \\
& \Rightarrow \stackrel{\text { : }}{\because}=-\delta \mu_{m} I_{1}^{4 b},
\end{aligned}
$$

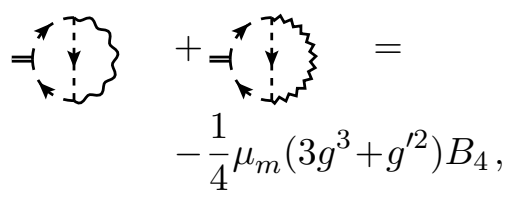

Singlet self-energy. The renormalised singlet self-energy $\hat{\Pi}_{\sigma \sigma}$ in Landau gauge composes of (excluding pure singlet terms eqs. (d.1)-(d.15))

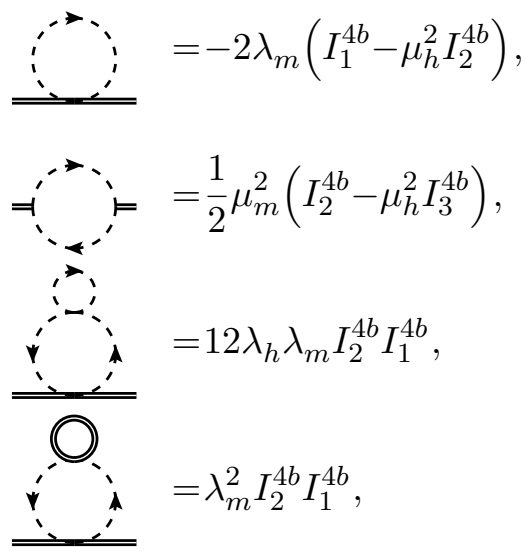

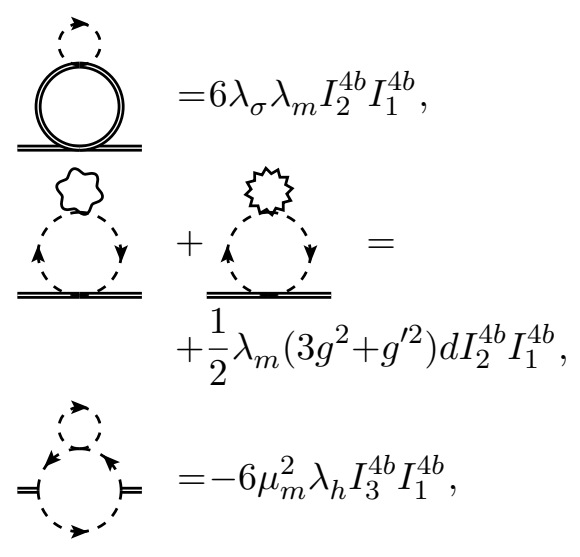




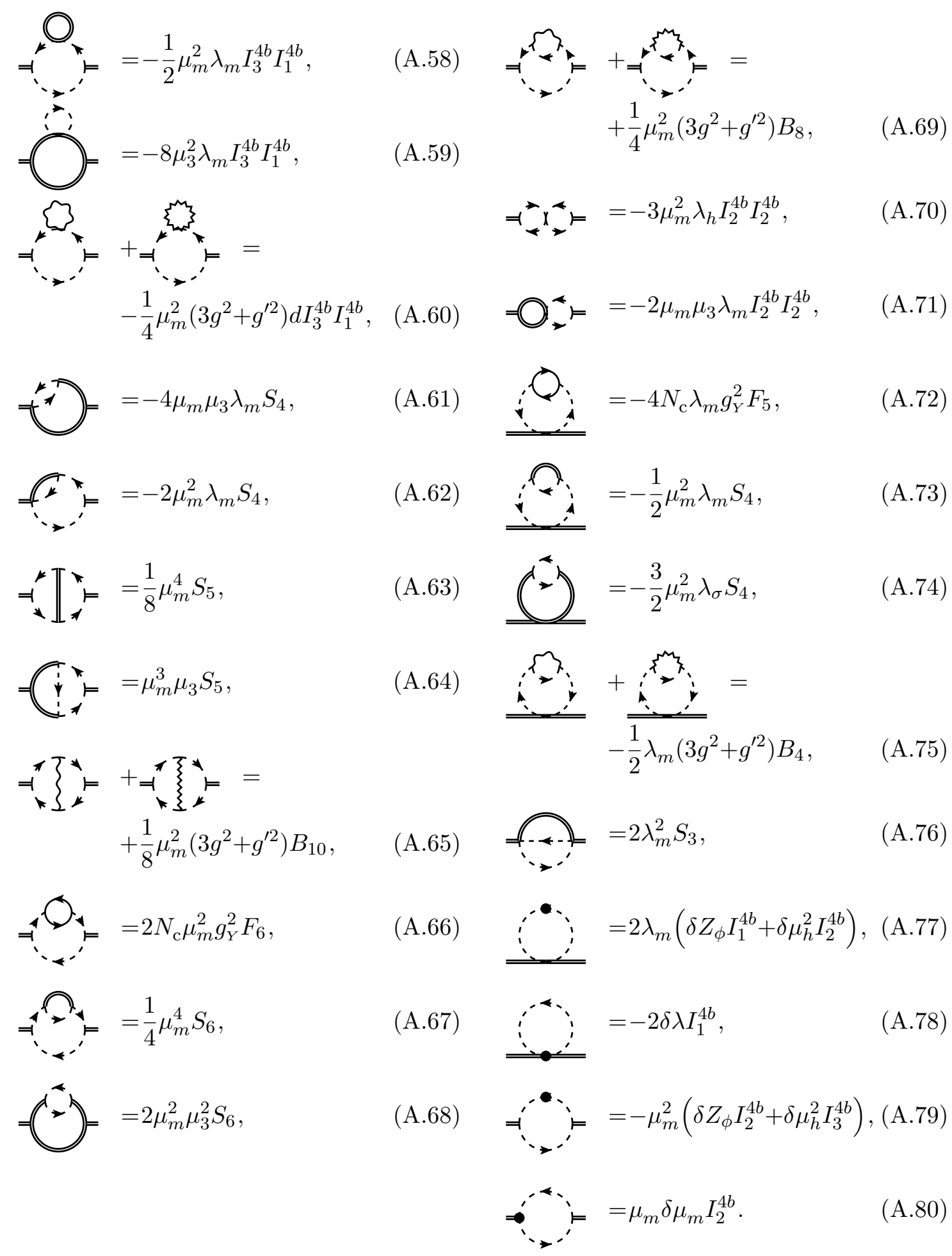

Higgs self-energy. The singlet contributions to the renormalised SM Higgs doublet selfenergy $\hat{\Pi}_{\phi^{\dagger} \phi}$ (in Landau gauge) read

$$
=-\frac{1}{2} \lambda_{m}\left(I_{1}^{4 b}-\mu_{\sigma}^{2} I_{2}^{4 b}\right),(\mathrm{A} .81) \quad=\frac{1}{4} \mu_{m}^{2}\left(I_{2}^{4 b}-\mu_{\sigma}^{2} I_{3}^{4 b}\right),
$$



ì $=3 \lambda_{h} \lambda_{m} I_{2}^{4 b} I_{1}^{4 b}$,
(A.83)
$=\lambda_{m}^{2} I_{2}^{4 b} I_{1}^{4 b}$
(A.84) $=\frac{1}{16} \mu_{m}^{4} S_{6}$,
$=\frac{3}{2} \lambda_{\sigma} \lambda_{m} I_{2}^{4 b} I_{1}^{4 b}$,
(A.85) $=\frac{1}{2} \mu_{m}^{2} \mu_{3}^{2} S_{6}$,
$\bigcup^{\pi}=-\frac{3}{2} \mu_{m}^{2} \lambda_{h} I_{3}^{4 b} I_{1}^{4 b}$,
(A.86)
$\overbrace{-1}^{\prime \prime}=-\frac{1}{2} \mu_{m}^{2} \lambda_{m} I_{3}^{4 b} I_{1}^{4 b}$

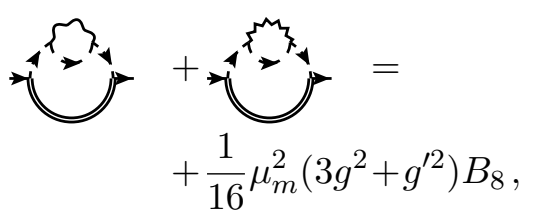
$\bigcup^{\pi}=-\frac{1}{8} \mu_{m}^{2} \lambda_{m} I_{3}^{4 b} I_{1}^{4 b}$,
(A.88)
$=-\frac{3}{4} \mu_{m}^{2} \lambda_{\sigma} I_{3}^{4 b} I_{1}^{4 b}$,
(A.89)
ר $=-\frac{1}{4} \mu_{m}^{2} \lambda_{m} I_{2}^{4 b} I_{2}^{4 b}$
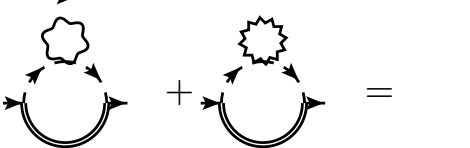$$
-\frac{1}{16} \mu_{m}^{2}\left(3 g^{2}+g^{\prime 2}\right) d I_{3}^{4 b} I_{1}^{4 b},
$$
(A.90)
$\because$
$\int^{4}=-\frac{1}{2} \mu_{m}^{2} \lambda_{m} S_{4}$,
ר:
$=-\frac{1}{4} \mu_{m}^{2} \lambda_{m} S_{4}$
入 $=-\mu_{m} \mu_{3} \lambda_{m} S_{4}$,
(A.93) $=-\frac{1}{2} \delta \lambda_{m} I_{1}^{4 b}$,
$\bigcirc_{-}^{2} \lambda_{m} S_{4}$
$\prod_{1}^{\pi}=\frac{1}{16} \mu_{m}^{4} S_{5}$
$\bigcap_{1}=\frac{1}{4} \mu_{m}^{3} \mu_{3} S_{5}$
唡 $=\frac{1}{2} N_{\mathrm{c}} \mu_{m}^{2} g_{Y}^{2} F_{6}$,

$$
\rightarrow=\frac{1}{2} \lambda_{m}^{2} S_{3},
$$$$
\bigcirc=\frac{1}{2} \lambda_{m}\left(\delta Z_{\sigma} I_{1}^{4 b}+\delta \mu_{\sigma}^{2} I_{2}^{4 b}\right),
$$
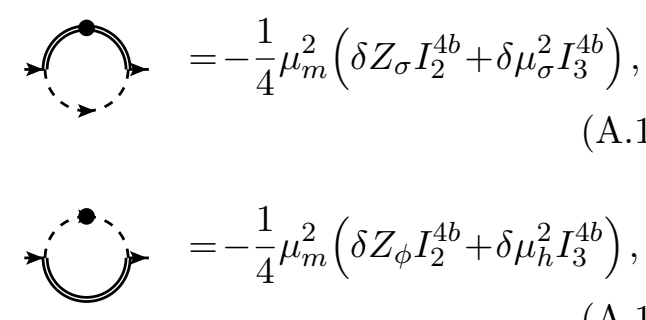

$$
\bigcap_{-1}=\frac{1}{2} \mu_{m} \delta \mu_{m} I_{2}^{4 b}
$$


Electroweak Debye masses and gauge couplings at two-loop. The singlet contributions to the gauge field self-energies are displayed in eqs. (4.55) and (4.56). Diagrammatically the $\mathrm{SU}(2)$ gauge field self-energy composes of

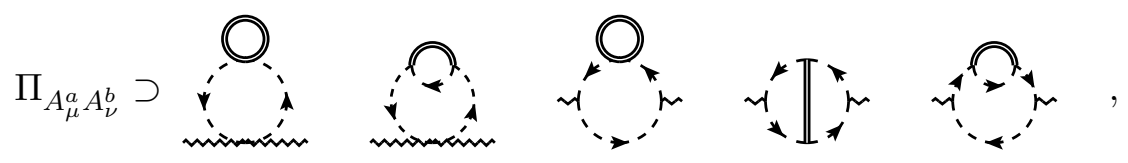

which is identical for $\mathrm{U}(1)$ when replacing the external legs: $\Pi_{A_{\mu}^{a} A_{\nu}^{b}} \rightarrow \Pi_{B_{\mu} B_{\nu}}$. Their corresponding SM contributions to the Debye mass align with refs. [129, 130]:

$$
\begin{aligned}
\left(m_{\mathrm{D}}^{\prime 2}\right)_{\mathrm{SM}}= & T^{2} g^{\prime 2} \frac{1}{24}\left(4 Y_{\phi}^{2}+Y_{2 \mathrm{f}} n_{\mathrm{f}}\right) \\
-\frac{T^{2}}{(4 \pi)^{2}}[ & \left(\frac{2 L_{b}-5}{72} Y_{\phi}^{4}+\frac{9 Y_{4 \mathrm{f}}+\left(L_{b}+4 L_{f}-2\right) Y_{2 \mathrm{f}} Y_{\phi}^{2}}{144} n_{\mathrm{f}}+\frac{\left(L_{f}-1\right)}{144}\left(Y_{2 \mathrm{f}} n_{\mathrm{f}}\right)^{2}\right) g^{\prime 4} \\
& -\frac{3}{8}\left(Y_{\phi}^{2}-\left(Y_{\ell}^{2}+N_{\mathrm{c}} Y_{q}^{2}\right) n_{\mathrm{f}}\right) g^{\prime 2} g^{2}-g^{\prime 2} \lambda_{h} Y_{\phi}^{2} \\
& \left.+\frac{2 Y_{q}^{2}+Y_{u}^{2}+Y_{d}^{2}}{4} C_{\mathrm{F}} N_{\mathrm{c}} n_{\mathrm{f}} g^{\prime 2} g_{\mathrm{s}}^{2}+\frac{6\left(Y_{q} Y_{u}-Y_{\phi}\left(Y_{q}-Y_{u}\right)\right)-5 Y_{\phi}^{2}}{12} N_{\mathrm{c}} g^{\prime 2} g_{Y}^{2}\right] \\
\left(m_{\mathrm{D}}^{2}\right)_{\mathrm{SM}}=T^{2} g^{2} \frac{1}{3}\left(\frac{5}{2}+\frac{N_{\mathrm{c}}+1}{4} n_{\mathrm{f}}\right) & \\
+\frac{T^{2}}{(4 \pi)^{2}}[ & \left(\frac{430 L_{b}+207}{72}+\frac{\left(N_{\mathrm{c}}+1\right)\left(43 L_{b}-20 L_{f}+11\right)}{72} n_{\mathrm{f}}\right. \\
& \left.-\frac{\left(N_{\mathrm{c}}+1\right)^{2}\left(L_{f}-1\right)}{36} n_{\mathrm{f}}^{2}\right) g^{4}+\frac{1}{8}\left(Y_{\phi}^{2}-\left(Y_{\ell}^{2}+N_{\mathrm{c}} Y_{q}^{2}\right) n_{\mathrm{f}}\right) g^{\prime 2} g^{2} \\
& \left.+g^{2} \lambda_{h} Y_{\phi}^{2}-\frac{1}{2} C_{\mathrm{F}} N_{\mathrm{c}} n_{\mathrm{f}} g^{2} g_{\mathrm{s}}^{2}-\frac{N_{\mathrm{c}}}{12} g^{2} g_{Y}^{2}\right] .
\end{aligned}
$$

Additionally, we show the two-loop singlet contributions to the gauge couplings:

$$
\begin{aligned}
g_{3}^{\prime 2} & =g^{\prime 4} T \frac{\zeta_{3}}{(4 \pi)^{4}} \frac{1}{36}\left[\lambda_{m}-\frac{1}{(4 \pi)^{2} T^{2}}\left(6 L_{b}+11\right) \mu_{m}^{2}\right]+(\text { SM terms }) \\
g_{3}^{2} & =g^{4} T \frac{\zeta_{3}}{(4 \pi)^{4}} \frac{1}{36}\left[\lambda_{m}-\frac{1}{(4 \pi)^{2} T^{2}}\left(6 L_{b}+11\right) \mu_{m}^{2}\right]+(\text { SM terms })
\end{aligned}
$$

These contributions are formally of higher order, i.e. $\mathcal{O}\left(g^{6}\right)$ in our power counting.

\section{B Collection of integrals}

This appendix collects definitions and results of sum-integrals encountered in our computation. Ref. [231] and references therein further showcase many explicit derivations. We use dimensional regularisation in $D=d+1=4-2 \epsilon$ dimensions in the $\overline{\mathrm{MS}}$-scheme with renormalisation scales $\Lambda$ in $4 \mathrm{~d}$ and $\Lambda_{3 \mathrm{~d}}$ in $3 \mathrm{~d}$. Euclidean four-momenta are denoted as $P \equiv\left(\omega_{n}, \mathbf{p}\right)$ with the bosonic Matsubara frequency $\omega_{n}=2 \pi n T$. We define the $d$-dimensional integral measure as

$$
\int_{p} \equiv\left(\frac{\Lambda^{2} e^{\gamma_{\mathrm{E}}}}{4 \pi}\right)^{\epsilon} \int \frac{\mathrm{d}^{d} p}{(2 \pi)^{d}}
$$


and bosonic sum-integrals as

$$
\mathcal{F}_{P} \equiv T \sum_{\omega_{n}} \int_{p}, \quad \mathcal{f}_{P}^{\prime} \equiv T \sum_{\omega_{n} \neq 0} \int_{p}
$$

where a primed integral denotes the absence of a zero mode. For fermionic counterparts, we employ the definition of ref. [151].

In pure $3 \mathrm{~d}$, we encounter the following integrals

$$
\begin{aligned}
J_{\text {soft }} & \equiv \frac{1}{2} \int_{p} \ln \left(p^{2}+m^{2}\right)=-\frac{1}{2}\left(\frac{\Lambda_{3 \mathrm{~d}}^{2} e^{\gamma_{\mathrm{E}}}}{4 \pi}\right)^{\epsilon} \frac{\left.{ }^{\epsilon} m^{2}\right]^{\frac{d}{2}}}{(4 \pi)^{\frac{d}{2}}} \frac{\Gamma\left(-\frac{d}{2}\right)}{\Gamma(1)} \\
& =-\frac{\left(m^{2}\right)^{\frac{3}{2}}}{12 \pi}+\mathcal{O}(\epsilon), \\
I_{\alpha}^{3}(m) & \equiv \int_{p} \frac{1}{\left[p^{2}+m^{2}\right]^{\alpha}}=\left(\frac{\Lambda_{3 \mathrm{~d}}^{2} e^{\gamma_{\mathrm{E}}}}{4 \pi}\right)^{\epsilon} \frac{\left[m^{2}\right]^{\frac{d}{2}-\alpha}}{(4 \pi)^{\frac{d}{2}}} \frac{\Gamma\left(\alpha-\frac{d}{2}\right)}{\Gamma(\alpha)}, \\
S_{3}^{3}\left(m_{1}, m_{2}, m_{3}\right) & \equiv \int_{p, q} \frac{1}{\left[p^{2}+m_{1}^{2}\right]\left[q^{2}+m_{2}^{2}\right]\left[(p+q)^{2}+m_{3}^{2}\right]} \\
& =\frac{1}{(4 \pi)^{2}}\left(\frac{1}{4 \epsilon}+\frac{1}{2}+\ln \left(\frac{\Lambda_{3 \mathrm{~d}}}{m_{1}+m_{2}+m_{3}}\right)\right)+\mathcal{O}(\epsilon),
\end{aligned}
$$

where we define the shorthand notation

$$
\begin{aligned}
\mathcal{D}_{S S}^{3 \mathrm{~d}}\left(m_{1}, m_{2}\right) & \equiv-I_{1}^{3}\left(m_{1}\right) I_{1}^{3}\left(m_{2}\right), \\
\mathcal{D}_{S S S}^{3 \mathrm{~d}}\left(m_{1}, m_{2}, m_{3}\right) & \equiv S_{3}^{3}\left(m_{1}, m_{2}, m_{3}\right) .
\end{aligned}
$$

In the $4 \mathrm{~d}$ computation, we encounter sum-integrals parameterised by

$$
\begin{aligned}
& Z_{s_{1} ; \sigma_{1}}^{\alpha_{1}} \equiv \oiint_{P\left\{\sigma_{1}\right\}} \frac{p_{0}^{\alpha_{1}}}{\left[P^{2}\right]^{s_{1}}}, \\
& Z_{s_{1} s_{2} s_{3} ; \sigma_{1} \sigma_{2}} \equiv \varlimsup_{P\left\{\sigma_{1}\right\} Q\left\{\sigma_{2}\right\}} \frac{1}{\left[P^{2}\right]^{s_{1}}\left[Q^{2}\right]^{s_{2}}\left[(P+Q)^{2}\right]^{s_{3}}} \text {, }
\end{aligned}
$$

where the Matsubara four-momenta have implicit fermion signature $P^{2}=\left[\left(2 n+\sigma_{i}\right) \pi T\right]^{2}+$ $\mathbf{p}^{2}$ with $\sigma_{i}=0(1)$ for bosons(fermions). Below we list some of the recurring integrals in 
$d=3-2 \epsilon$

$$
\begin{aligned}
& Z_{\alpha ; 0}=I_{\alpha}^{4 b} \equiv \oiint_{P}^{\prime} \frac{1}{\left[P^{2}\right]^{\alpha}}=\left(\frac{\Lambda^{2} e^{\gamma_{\mathrm{E}}}}{4 \pi}\right)^{\epsilon} 2 T \frac{[2 \pi T]^{d-2 \alpha}}{(4 \pi)^{\frac{d}{2}}} \frac{\Gamma\left(\alpha-\frac{d}{2}\right)}{\Gamma(\alpha)} \zeta_{2 \alpha-d}, \\
& Z_{\alpha ; 1}=I_{\alpha}^{4 f} \equiv \oiint_{\{P\}} \frac{1}{\left[P^{2}\right]^{\alpha}}=\left(2^{2 \alpha-d}-1\right) I_{\alpha}^{4 b}, \\
& Z_{111 ; 00}=S_{3} \equiv \varlimsup_{P, Q} \frac{1}{P^{2} Q^{2}(P+Q)^{2}}=0, \\
& Z_{211 ; 00}=S_{4} \equiv \oiint_{P, Q} \frac{1}{P^{4} Q^{2}(P+Q)^{2}}=-\frac{1}{(d-5)(d-2)} I_{2}^{4 b} I_{2}^{4 b}, \\
& Z_{221 ; 00}=S_{5} \equiv \oiint_{P, Q} \frac{1}{P^{4} Q^{4}(P+Q)^{2}}=0 \text {, } \\
& Z_{311 ; 00}=S_{6} \equiv \oiint_{P, Q} \frac{1}{P^{6} Q^{2}(P+Q)^{2}}=-\frac{4}{(d-7)(d-2)} I_{3}^{4 b} I_{2}^{4 b} \\
& Z_{121 ; 10}=\mathcal{F} \equiv \varlimsup_{\{P\}, Q} \frac{1}{P^{2} Q^{4}(P+Q)^{2}}=\frac{1}{(d-5)(d-2)}\left(I_{2}^{4 f} I_{2}^{4 f}-2 I_{2}^{4 f} I_{2}^{4 b}\right) \text {, } \\
& F_{5} \equiv \varlimsup_{\{P\}, Q} \frac{P^{2}+P \cdot Q}{P^{2} Q^{4}(P+Q)^{2}}=\left(2^{2-d}-1\right) I_{2}^{4 b} I_{1}^{4 b} \text {, } \\
& F_{6} \equiv \mathcal{F}_{\{P\}, Q} \frac{P^{2}+P \cdot Q}{P^{2} Q^{6}(P+Q)^{2}}=I_{1}^{4 f} I_{3}^{4 b}-\frac{1}{2} \mathcal{F} \text {, } \\
& B_{4} \equiv \varlimsup_{P, Q} \frac{1}{P^{4}(P+Q)^{2}}(2 P+Q)_{\mu}(2 P+Q)_{\nu} D_{\mu \nu}(Q)=0, \\
& B_{8} \equiv \oiint_{P, Q} \frac{1}{P^{6} Q^{2}}(P-Q)_{\mu}(P-Q)_{\nu} D_{\mu \nu}(P+Q)=I_{2}^{4 b} I_{2}^{4 b}+S_{4}, \\
& B_{10} \equiv \oiint_{P, Q} \frac{1}{P^{4} Q^{4}}(P-Q)_{\mu}(P-Q)_{\nu} D_{\mu \nu}(P+Q)=4 S_{4}-3 I_{2}^{4 b} I_{2}^{4 b} .
\end{aligned}
$$

Here the power of integration-by-parts reduction (cf. ref. [224] and in particular section 3.4 of ref. [171]) is obvious. All massless two-loop sum-integrals reduce to one-loop masters. Similar sum-integral structures are listed in appendix C of ref. [157] and can be used to compute pure SM contributions to the Higgs self energy at two-loop. In broken phase computations, we need massive sum-integrals (cf. ref. [111]), expanded at high- $T$ setting 
$m / T \ll 1$

$$
\begin{aligned}
& J_{b, \text { hard }}(m) \equiv \frac{1}{2} \oint_{P}^{\prime} \ln \left(P^{2}+m^{2}\right) \\
& \simeq m^{2} \frac{1}{2} I_{1}^{4 b}-\frac{1}{4} m^{4} I_{2}^{4 b}+\frac{1}{6} m^{6} I_{3}^{4 b}-\frac{1}{8} m^{8} I_{4}^{4 b}+\frac{1}{10} m^{10} I_{5}^{4 b}-\frac{1}{12} m^{12} I_{6}^{4 b} \\
& =\frac{T^{2}}{24} m^{2}-\frac{1}{(4 \pi)^{2}} \frac{m^{4}}{4}\left(\frac{1}{\epsilon}+L_{b}\right)+\frac{\zeta_{3}}{3(4 \pi)^{4}} \frac{m^{6}}{T^{2}}-\frac{\zeta_{5}}{2(4 \pi)^{6}} \frac{m^{8}}{T^{4}} \\
& +\frac{\zeta_{7}}{(4 \pi)^{8}} \frac{m^{10}}{T^{6}}-\frac{7}{3} \frac{\zeta_{9}}{(4 \pi)^{10}} \frac{m^{12}}{T^{8}}+\mathcal{O}(\epsilon), \\
& J_{f}(m) \equiv \frac{1}{2} \sum_{\{P\}} \ln \left(P^{2}+m^{2}\right) \\
& \simeq \frac{1}{2} m^{2} I_{1}^{4 f}-\frac{1}{4} m^{4} I_{2}^{4 f}+\frac{1}{6} m^{6} I_{3}^{4 f} \\
& =-\frac{T^{2}}{48} m^{2}-\frac{1}{(4 \pi)^{2}} \frac{m^{4}}{4}\left(\frac{1}{\epsilon}+L_{b}\right)+\frac{7}{3} \frac{\zeta_{3}}{(4 \pi)^{4}} \frac{m^{6}}{T^{2}}+\mathcal{O}(\epsilon), \\
& \mathcal{D}_{S S}\left(m_{1}, m_{2}\right) \equiv-\oiint_{P} \frac{1}{P^{2}+m_{1}^{2}} \Varangle_{Q} \frac{1}{Q^{2}+m_{2}^{2}} \\
& =-\left(T^{2} I_{1}^{3}\left(m_{1}\right) I_{1}^{3}\left(m_{2}\right)+I_{1}^{4 b} I_{1}^{4 b}\right. \\
& +I_{1}^{4 b}\left(-\left(m_{1}^{2}+m_{2}^{2}\right) I_{2}^{4 b}+T I_{1}^{3}\left(m_{1}\right)+T I_{1}^{3}\left(m_{2}\right)\right) \\
& \left.-I_{2}^{4 b} T\left(m_{1}^{2} I_{1}^{3}\left(m_{2}\right)+m_{2}^{2} I_{1}^{3}\left(m_{1}\right)\right)+m_{1}^{2} m_{1}^{2} I_{2}^{4 b} I_{2}^{4 b}+\left(m_{1}^{4}+m_{2}^{4}\right) I_{1}^{4 b} I_{3}^{4 b}\right) \\
& +\mathcal{O}\left(m^{5}\right) \text {, } \\
& \mathcal{D}_{S S S}\left(m_{1}, m_{2}, m_{3}\right) \equiv \oiint_{P, Q} \frac{1}{\left[P^{2}+m_{1}^{2}\right]\left[Q^{2}+m_{2}^{2}\right]\left[(P+Q)^{2}+m_{3}^{2}\right]} \\
& =T^{2} S_{3}^{3}\left(m_{1}, m_{2}, m_{3}\right)+T\left(I_{1}^{3}\left(m_{1}\right)+I_{1}^{3}\left(m_{2}\right)+I_{1}^{3}\left(m_{3}\right)\right) I_{2}^{4 b} \\
& -\left(m_{1}^{2}+m_{2}^{2}+m_{3}^{2}\right) S_{4}+\left(m_{1}^{4}+m_{2}^{4}+m_{3}^{4}\right)\left(S_{5}+S_{6}\right) \\
& +\mathcal{O}\left(m^{3}\right)+\mathcal{O}\left(m^{5}\right) \\
& \mathcal{D}_{S}\left(m, \delta m^{2}, \delta Z\right) \equiv \oiint_{P} \frac{\left(\delta m^{2}+P^{2} \delta Z\right)}{P^{2}+m^{2}} \\
& =\left(\delta m^{2}-m^{2} \delta Z\right)\left(T I_{1}^{3}(m)+I_{1}^{4 b}-m^{2} I_{2}^{4 b}\right)+\mathcal{O}\left(m^{4}\right) .
\end{aligned}
$$

Note that if one uses resummed propagators, all $3 \mathrm{~d}$ integrals in these expressions obtain a resummed $3 \mathrm{~d}$ mass instead of a $4 \mathrm{~d}$ mass. In $\mathcal{D}_{S S}$ and $\mathcal{D}_{S S S}$ all mixed soft/hard terms (that are non-analytic in $m^{2}$ ) will be cancelled in resummation at $\mathcal{O}\left(g^{4}\right)$. For the latter integral, we do not write down terms of $\mathcal{O}\left(\mathrm{m}^{3}\right)$ explicitly since these are absent in resummation at $\mathcal{O}\left(g^{4}\right)$. Whereas the $\mathcal{O}\left(m^{4}\right)$ hard contribution is required for the matching of $\mu_{3}^{4}$ terms. Indeed, in addition to $\mathcal{O}\left(\mathrm{m}^{2}\right)$ terms we need $\mathcal{O}\left(\mathrm{m}^{4}\right)$ terms due to cubic interaction $\mu_{3}$ which can be compared eqs. (81) and (88) of the classic ref. [111]. 
Sunset sum-integrals. Finally, let us inspect the high- $T$ expansion of the massive twoloop sunset sum-integral. See also ref. [237]. The result given in (B.24) shows almost surprising cleanliness in the terms of the mass expansion. In particular, it is very pleasant that the coefficients of even powers of mass are given in terms of full sum-integral structures, as opposed to linear combinations of (massless) mixed soft/hard and hard sum-integral structures. Essentially, this can be shown to take place by carefully expanding separately the mixed modes and hard modes of the original massive sum-integral. The arithmetic challenge arises from a proper treatment of the mixed modes, as a naive mass expansion can only occur in propagators with non-vanishing Matsubara index (hard scale contribution). Hence, the proper order of the mass expansion is chosen to be isolated with an iterative approach. This operation is explicitly described below for the special case with three degenerate masses. This simplifies the book keeping of the computation without any loss of information, due to the obvious symmetry between the three masses.

It is well-motivated to symmetrise the computation as far as possible. Thus, we choose to consider the hard modes by setting each propagator structure of the sunset integral identical, with non-vanishing thermal component:

$$
D_{S S S}^{\text {hard }}=\oiint_{P, Q} \frac{\left(1-\delta_{P_{0}}\right)\left(1-\delta_{Q_{0}}\right)\left(1-\delta_{P_{0}+Q_{0}}\right)}{\left(P^{2}+m^{2}\right)\left(Q^{2}+m^{2}\right)\left[(P+Q)^{2}+m^{2}\right]} .
$$

We can expand this in a trivial manner up to $\mathcal{O}\left(\mathrm{m}^{2}\right)$ to find the following non-vanishing contributions:

$$
-3 T \int_{p} \oiint_{Q} \frac{1}{p^{2} Q^{2}(p+Q)^{2}}-3 m^{2}\left[\oiint_{P Q} \frac{\left(1-\delta_{P_{0}}\right)\left(1-\delta_{Q_{0}}\right)}{P^{4} Q^{2}(P+Q)^{2}}-T \int_{p} \oiint_{Q} \frac{\left(1-\delta_{Q_{0}}\right)}{p^{2} Q^{4}(p+Q)^{2}}\right],
$$

where we took note that all scaleless spatial integrals vanish, and more notably the massless sunset integral $S_{3}$ (B.11) vanishes [230, 238]. The first integral expression mixes soft and hard modes. This naturally follows from the symmetrisation of the propagators, which essentially adds contributions from the mixed part of the expansion. Hence, we reinsert it in the second structure of interest.

$$
D_{S S S}^{\mathrm{mix}}=3 T \int_{p} \oiint_{Q} \frac{1-\delta_{Q_{0}}}{\left(p^{2}+m^{2}\right)\left(Q^{2}+m^{2}\right)\left[(p+Q)^{2}+m^{2}\right]}-3 T \int_{p} \oiint_{Q} \frac{1}{p^{2} Q^{2}(p+Q)^{2}}
$$

Let us start the expansion towards $\mathcal{O}\left(\mathrm{m}^{2}\right)$ by solely considering the propagators with nonvanishing thermal scale, which yields

$$
\begin{aligned}
& 3 T \int_{p} \mathcal{F}_{Q} \frac{\left(1-\delta_{Q_{0}}\right)}{\left(p^{2}+m^{2}\right) Q^{2}(p+Q)^{2}}-3 T \int_{p} \mathcal{F}_{Q} \frac{1}{p^{2} Q^{2}(p+Q)^{2}}-6 T m^{2} \int_{p} \mathcal{F}_{Q} \frac{1-\delta_{Q_{0}}}{\left(p^{2}+m^{2}\right) Q^{4}(p+Q)^{2}} \\
& =-3 T m^{2} \int_{p} \mathcal{F}_{Q}\left[\frac{1-\delta_{Q_{0}}}{p^{2}\left(p^{2}+m^{2}\right) Q^{2}(p+Q)^{2}}+\frac{2\left(1-\delta_{Q_{0}}\right)}{\left(p^{2}+m^{2}\right) Q^{4}(p+Q)^{2}}\right] .
\end{aligned}
$$

In both of these sum-integral terms it suffices to remove the mass terms inside the integral, as we only wish to find contributions exactly at $\mathcal{O}\left(\mathrm{m}^{2}\right)$. Thus, we find the quadratic 
coefficient of the expansion as

$$
\begin{aligned}
D_{S S S}^{m^{2}} & =-3\left[\int_{P Q} \frac{\left(1-\delta_{P_{0}}\right)\left(1-\delta_{Q_{0}}\right)}{P^{4} Q^{2}(P+Q)^{2}}+T \int_{p} \mathcal{\gamma}_{Q} \frac{1-\delta_{Q_{0}}}{p^{4} Q^{2}(p+Q)^{2}}+T \int_{p} \gamma_{Q} \frac{2\left(1-\delta_{Q_{0}}\right)}{\left[p^{2}+m^{2}\right] Q^{4}(p+Q)^{2}}\right] \\
& \equiv-3 S_{4},
\end{aligned}
$$

where $S_{4}$ is defined by eq. (B.12).

We follow a similar procedure to find the coefficient of $\mathcal{O}\left(\mathrm{m}^{4}\right)$, with the slight difference of using the results found above as the iterative subtraction element for the mixed element expansion. This time, the hard expansion yields

$$
\begin{aligned}
& 3 m^{4} \oiint_{P Q}\left[\frac{\left(1-\delta_{P_{0}}\right)\left(1-\delta_{Q_{0}}\right)\left(1-\delta_{P_{0}+Q_{0}}\right)}{P^{6} Q^{2}(P+Q)^{2}}+\frac{\left(1-\delta_{P_{0}}\right)\left(1-\delta_{Q_{0}}\right)\left(1-\delta_{P_{0}+Q_{0}}\right)}{P^{4} Q^{4}(P+Q)^{2}}\right] \\
& =3 m^{4}\left[\oiint_{P Q} \frac{\left(1-\delta_{P_{0}}\right)\left(1-\delta_{Q_{0}}\right)}{P^{6} Q^{2}(P+Q)^{2}}-T \int_{p} \oiint_{Q} \frac{1-\delta_{Q_{0}}}{p^{2} Q^{6}(p+Q)^{2}}\right] \\
& +3 m^{4}\left[\oint_{P Q} \frac{\left(1-\delta_{P_{0}}\right)\left(1-\delta_{Q_{0}}\right)}{P^{4} Q^{2}(P+Q)^{4}}-T \int_{p} \oiint_{Q} \frac{1-\delta_{Q_{0}}}{p^{4} Q^{2}(p+Q)^{4}}\right] .
\end{aligned}
$$

In order to fully extract the mixed contributions, we again expand the suitable propagators and follow-up with a removal of the results of previous orders. This results in three separate computations:

$$
\begin{aligned}
3 T \int_{p} \oiint_{Q}\left[\frac{\left(1-\delta_{Q_{0}}\right)}{\left(p^{2}+m^{2}\right) Q^{2}(p+Q)^{2}}-\frac{\left(1-\delta_{Q_{0}}\right)}{p^{2} Q^{2}(p+Q)^{2}}+\frac{m^{2}}{p^{4} Q^{2}(p+Q)^{2}}\right] \\
\mapsto 3 m^{4} T \int_{p} \oiint_{Q} \frac{\left(1-\delta_{Q_{0}}\right)}{p^{6} Q^{2}(p+Q)^{2}}, \\
\quad-6 T m^{2} \int_{p} \oiint_{Q}\left[\frac{\left(1-\delta_{Q_{0}}\right)}{\left(p^{2}+m^{2}\right) Q^{4}(p+Q)^{2}}-\frac{\left(1-\delta_{Q_{0}}\right)}{p^{2} Q^{4}(p+Q)^{2}}\right] \\
\mapsto 6 T m^{4} \int_{p} \oiint_{Q} \frac{\left(1-\delta_{Q_{0}}\right)}{p^{4} Q^{2}(p+Q)^{4}}, \\
3 T m^{4} \int_{p} \oiint_{Q}\left[\frac{2\left(1-\delta_{Q_{0}}\right)}{\left(p^{2}+m^{2}\right) Q^{6}(p+Q)^{2}}+\frac{\left(1-\delta_{Q_{0}}\right)}{\left(p^{2}+m^{2}\right) Q^{4}(p+Q)^{4}}\right] \\
\mapsto 3 m^{4} T \int_{p} \oiint_{Q}\left[\frac{2\left(1-\delta_{Q_{0}}\right)}{p^{2} Q^{6}(p+Q)^{2}}+\frac{\left(1-\delta_{Q_{0}}\right)}{p^{2} Q^{4}(p+Q)^{4}}\right] .
\end{aligned}
$$

These contributions combine to the $\mathcal{O}\left(\mathrm{m}^{4}\right)$ coefficient of the mass expansion as

$$
D_{S S S}^{m^{4}}=3\left[\oiint_{P Q} \frac{1}{P^{6} Q^{2}(P+Q)^{2}}+\frac{1}{P^{4} Q^{2}(P+Q)^{4}}\right] \equiv 3\left[S_{5}+S_{6}\right],
$$

where $S_{5}$ is defined in eq. (B.13) and $S_{6}$ in (B.14).

Open Access. This article is distributed under the terms of the Creative Commons Attribution License (CC-BY 4.0), which permits any use, distribution and reproduction in any medium, provided the original author(s) and source are credited. 


\section{References}

[1] R. Apreda, M. Maggiore, A. Nicolis and A. Riotto, Gravitational waves from electroweak phase transitions, Nucl. Phys. B 631 (2002) 342 [gr-qc/0107033] [InSPIRE].

[2] C. Grojean and G. Servant, Gravitational waves from phase transitions at the electroweak scale and beyond, Phys. Rev. D 75 (2007) 043507 [hep-ph/0607107] [INSPIRE].

[3] D.J. Weir, Gravitational waves from a first order electroweak phase transition: a brief review, Phil. Trans. Roy. Soc. Lond. A 376 (2018) 20170126 [arXiv:1705.01783] [INSPIRE].

[4] C. Caprini and D.G. Figueroa, Cosmological backgrounds of gravitational waves, Class. Quant. Grav. 35 (2018) 163001 [arXiv:1801.04268] [INSPIRE].

[5] M.B. Hindmarsh, M. Lüben, J. Lumma and M. Pauly, Phase transitions in the early universe, SciPost Phys. Lect. Notes 24 (2021) 1 [arXiv:2008.09136] [InSPIRE].

[6] LIGO SCIENTIFIC and VIRGO collaborations, Observation of gravitational waves from a binary black hole merger, Phys. Rev. Lett. 116 (2016) 061102 [arXiv:1602.03837] [INSPIRE].

[7] LIGO ScIEnTIFIC and VIRGo collaborations, GW151226: observation of gravitational waves from a 22-solar-mass binary black hole coalescence, Phys. Rev. Lett. 116 (2016) 241103 [arXiv:1606.04855] [INSPIRE].

[8] LIGO SCIENTIFIC and VIRGO collaborations, GW170104: observation of a 50-solar-mass binary black hole coalescence at redshift 0.2, Phys. Rev. Lett. 118 (2017) 221101 [Erratum ibid. 121 (2018) 129901] [arXiv:1706.01812] [INSPIRE].

[9] LIGO SCIENTIFIC and VIRGo collaborations, GW170817: observation of gravitational waves from a binary neutron star inspiral, Phys. Rev. Lett. 119 (2017) 161101 [arXiv:1710.05832] [INSPIRE].

[10] Ligo Scientific, Virgo, Fermi GBM, integral, IceCube, Astrosat Cadmium Zinc Telluride Imager Team, IPN, Insight-Hxmt, ANTARES, Swift, AGile Team, 1M2H Team, Dark Energy Camera GW-EM, DES, DLT40, GRAWITA, Fermi-LAT, ATCA, ASKAP, Las Cumbres Observatory Group, OzGrav, DWF (Deeper Wider Faster Program), ASt3, CAAstro, Vinrouge, MASter, J-GEM, GROWTH, JAGWAR, CALTEChNRAO, TTU-NRAO, NuSTAR, PAn-STARrS, MAXI Team, TZAC Consortium, KU, Nordic Optical Telescope, ePESSTO, GROND, Texas Tech University, SALt Group, TOROS, BOOTES, MWA, CALET, IKI-GW Follow-UP, H.E.S.S., LOFAR, LWA, HAWC, Pierre Auger, Alma, Euro Vlbi Team, Pi of Sky, Chandra Team at McGill University, DFN, ATlas Telescopes, High Time Resolution Universe Survey, RIMAS, RATIR and SKA South AFRICA/MEERKAT collaborations, Multi-messenger observations of a binary neutron star merger, Astrophys. J. Lett. 848 (2017) L12 [arXiv:1710.05833] [INSPIRE].

[11] LigO Scientific, Virgo, Fermi-GBM and INTEGRAL collaborations, Gravitational waves and gamma-rays from a binary neutron star merger: GW170817 and GRB 170817 A, Astrophys. J. Lett. 848 (2017) L13 [arXiv:1710.05834] [INSPIRE].

[12] LISA collaboration, Laser Interferometer Space Antenna, arXiv:1702.00786 [INSPIRE]. 
[13] G.M. Harry, P. Fritschel, D.A. Shaddock, W. Folkner and E.S. Phinney, Laser interferometry for the big bang observer, Class. Quant. Grav. 23 (2006) 4887 [Erratum ibid. 23 (2006) 7361] [INSPIRE].

[14] W.-H. Ruan, Z.-K. Guo, R.-G. Cai and Y.-Z. Zhang, Taiji program: gravitational-wave sources, Int. J. Mod. Phys. A 35 (2020) 2050075 [arXiv:1807.09495] [INSPIRE].

[15] S. Kawamura et al., The Japanese space gravitational wave antenna: DECIGO, Class. Quant. Grav. 28 (2011) 094011 [inSPIRE].

[16] A. Ashoorioon and T. Konstandin, Strong electroweak phase transitions without collider traces, JHEP 07 (2009) 086 [arXiv:0904.0353] [INSPIRE].

[17] A. Alves, T. Ghosh, H.-K. Guo, K. Sinha and D. Vagie, Collider and gravitational wave complementarity in exploring the singlet extension of the Standard Model, JHEP 04 (2019) 052 [arXiv: 1812.09333] [INSPIRE].

[18] A. Mazumdar and G. White, Review of cosmic phase transitions: their significance and experimental signatures, Rept. Prog. Phys. 82 (2019) 076901 [arXiv:1811.01948] [INSPIRE].

[19] K. Hashino, R. Jinno, M. Kakizaki, S. Kanemura, T. Takahashi and M. Takimoto, Selecting models of first-order phase transitions using the synergy between collider and gravitational-wave experiments, Phys. Rev. D 99 (2019) 075011 [arXiv:1809.04994] [INSPIRE].

[20] K. Kajantie, M. Laine, K. Rummukainen and M.E. Shaposhnikov, Is there a hot electroweak phase transition at $m_{H} \gtrsim m_{W}$ ?, Phys. Rev. Lett. 77 (1996) 2887 [hep-ph/9605288] [INSPIRE].

[21] K. Kajantie, M. Laine, K. Rummukainen and M.E. Shaposhnikov, A nonperturbative analysis of the finite $T$ phase transition in $\mathrm{SU}(2) \times \mathrm{U}(1)$ electroweak theory, Nucl. Phys. B 493 (1997) 413 [hep-lat/9612006] [INSPIRE].

[22] M. Gürtler, E.-M. Ilgenfritz and A. Schiller, Where the electroweak phase transition ends, Phys. Rev. D 56 (1997) 3888 [hep-lat/9704013] [INSPIRE].

[23] F. Csikor, Z. Fodor and J. Heitger, Endpoint of the hot electroweak phase transition, Phys. Rev. Lett. 82 (1999) 21 [hep-ph/9809291] [INSPIRE].

[24] M. D'Onofrio and K. Rummukainen, Standard Model cross-over on the lattice, Phys. Rev. D 93 (2016) 025003 [arXiv:1508.07161] [InSPIRE].

[25] ATLAS collaboration, Observation of a new particle in the search for the Standard Model Higgs boson with the ATLAS detector at the LHC, Phys. Lett. B 716 (2012) 1 [arXiv:1207.7214] [INSPIRE].

[26] CMS collaboration, Observation of a new boson at a mass of $125 \mathrm{GeV}$ with the CMS experiment at the LHC, Phys. Lett. B $\mathbf{7 1 6}$ (2012) 30 [arXiv:1207.7235] [INSPIRE].

[27] V.A. Kuzmin, V.A. Rubakov and M.E. Shaposhnikov, On the anomalous electroweak baryon number nonconservation in the early universe, Phys. Lett. B 155 (1985) 36 [INSPIRE].

[28] M. Trodden, Electroweak baryogenesis, Rev. Mod. Phys. 71 (1999) 1463 [hep-ph/9803479] [INSPIRE].

[29] D.E. Morrissey and M.J. Ramsey-Musolf, Electroweak baryogenesis, New J. Phys. 14 (2012) 125003 [arXiv:1206.2942] [INSPIRE]. 
[30] M.J. Ramsey-Musolf, The electroweak phase transition: a collider target, JHEP 09 (2020) 179 [arXiv: 1912.07189] [INSPIRE].

[31] J.M. No and M. Ramsey-Musolf, Probing the Higgs portal at the LHC through resonant di-Higgs production, Phys. Rev. D 89 (2014) 095031 [arXiv:1310.6035] [INSPIRE].

[32] G.C. Dorsch, S.J. Huber, K. Mimasu and J.M. No, Echoes of the electroweak phase transition: discovering a second Higgs doublet through $A_{0} \rightarrow Z H_{0}$, Phys. Rev. Lett. 113 (2014) 211802 [arXiv:1405.5537] [INSPIRE].

[33] N. Craig, H.K. Lou, M. McCullough and A. Thalapillil, The Higgs portal above threshold, JHEP 02 (2016) 127 [arXiv: 1412.0258] [INSPIRE].

[34] A.V. Kotwal, M.J. Ramsey-Musolf, J.M. No and P. Winslow, Singlet-catalyzed electroweak phase transitions in the $100 \mathrm{TeV}$ frontier, Phys. Rev. D 94 (2016) 035022 [arXiv: 1605.06123] [INSPIRE].

[35] T. Huang et al., Resonant di-Higgs boson production in the $b \bar{b} W W$ channel: probing the electroweak phase transition at the LHC, Phys. Rev. D 96 (2017) 035007 [arXiv: 1701.04442] [INSPIRE].

[36] C.-Y. Chen, J. Kozaczuk and I.M. Lewis, Non-resonant collider signatures of a singlet-driven electroweak phase transition, JHEP 08 (2017) 096 [arXiv:1704.05844] [INSPIRE].

[37] N.F. Bell, M.J. Dolan, L.S. Friedrich, M.J. Ramsey-Musolf and R.R. Volkas, Two-step electroweak symmetry-breaking: theory meets experiment, JHEP 05 (2020) 050 [arXiv: 2001.05335] [INSPIRE].

[38] Y. Kondo, I. Umemura and K. Yamamoto, First order phase transition in the singlet Majoron model, Phys. Lett. B 263 (1991) 93 [INSPIRE].

[39] K. Enqvist, K. Kainulainen and I. Vilja, Phase transitions in the singlet Majoron model, Nucl. Phys. B 403 (1993) 749 [INSPIRE].

[40] J.R. Espinosa and M. Quirós, The electroweak phase transition with a singlet, Phys. Lett. B 305 (1993) 98 [hep-ph/9301285] [INSPIRE].

[41] J. Choi and R.R. Volkas, Real Higgs singlet and the electroweak phase transition in the Standard Model, Phys. Lett. B 317 (1993) 385 [hep-ph/9308234] [InSPIRE].

[42] S. Profumo, M.J. Ramsey-Musolf and G. Shaughnessy, Singlet Higgs phenomenology and the electroweak phase transition, JHEP 08 (2007) 010 [arXiv:0705.2425] [INSPIRE].

[43] A. Ahriche, What is the criterion for a strong first order electroweak phase transition in singlet models?, Phys. Rev. D 75 (2007) 083522 [hep-ph/0701192] [INSPIRE].

[44] J.R. Espinosa, T. Konstandin and F. Riva, Strong electroweak phase transitions in the Standard Model with a singlet, Nucl. Phys. B 854 (2012) 592 [arXiv:1107.5441] [INSPIRE].

[45] J.M. Cline and K. Kainulainen, Electroweak baryogenesis and dark matter from a singlet Higgs, JCAP 01 (2013) 012 [arXiv:1210.4196] [INSPIRE].

[46] J.M. Cline, K. Kainulainen, P. Scott and C. Weniger, Update on scalar singlet dark matter, Phys. Rev. D 88 (2013) 055025 [Erratum ibid. 92 (2015) 039906] [arXiv:1306.4710] [INSPIRE]. 
[47] T. Alanne, K. Tuominen and V. Vaskonen, Strong phase transition, dark matter and vacuum stability from simple hidden sectors, Nucl. Phys. B $\mathbf{8 8 9}$ (2014) 692 [arXiv: 1407.0688] [INSPIRE].

[48] S. Profumo, M.J. Ramsey-Musolf, C.L. Wainwright and P. Winslow, Singlet-catalyzed electroweak phase transitions and precision Higgs boson studies,

Phys. Rev. D 91 (2015) 035018 [arXiv:1407.5342] [InSPIRE].

[49] D. Curtin, P. Meade and C.-T. Yu, Testing electroweak baryogenesis with future colliders, JHEP 11 (2014) 127 [arXiv:1409.0005] [INSPIRE].

[50] M. Kakizaki, S. Kanemura and T. Matsui, Gravitational waves as a probe of extended scalar sectors with the first order electroweak phase transition, Phys. Rev. D 92 (2015) 115007 [arXiv: 1509.08394] [INSPIRE].

[51] A. Beniwal, M. Lewicki, J.D. Wells, M. White and A.G. Williams, Gravitational wave, collider and dark matter signals from a scalar singlet electroweak baryogenesis, JHEP 08 (2017) 108 [arXiv: 1702.06124] [INSPIRE].

[52] G. Kurup and M. Perelstein, Dynamics of electroweak phase transition in singlet-scalar extension of the Standard Model, Phys. Rev. D 96 (2017) 015036 [arXiv:1704.03381] [INSPIRE].

[53] C.-W. Chiang, M.J. Ramsey-Musolf and E. Senaha, Standard Model with a complex scalar singlet: cosmological implications and theoretical considerations, Phys. Rev. D 97 (2018) 015005 [arXiv: 1707.09960] [InSPIRE].

[54] C.-W. Chiang, Y.-T. Li and E. Senaha, Revisiting electroweak phase transition in the Standard Model with a real singlet scalar, Phys. Lett. B $\mathbf{7 8 9}$ (2019) 154 [arXiv: 1808.01098] [INSPIRE].

[55] T. Alanne, T. Hugle, M. Platscher and K. Schmitz, A fresh look at the gravitational-wave signal from cosmological phase transitions, JHEP 03 (2020) 004 [arXiv: 1909.11356] [INSPIRE].

[56] N. Chen, T. Li, Y. Wu and L. Bian, Complementarity of the future $e^{+} e^{-}$colliders and gravitational waves in the probe of complex singlet extension to the Standard Model, Phys. Rev. D 101 (2020) 075047 [arXiv:1911.05579] [InSPIRE].

[57] T. Alanne et al., Pseudo-Goldstone dark matter: gravitational waves and direct-detection blind spots, JHEP 10 (2020) 080 [arXiv: 2008.09605] [INSPIRE].

[58] A. Papaefstathiou and G. White, The electro-weak phase transition at colliders: confronting theoretical uncertainties and complementary channels, JHEP 05 (2021) 099 [arXiv: 2010.00597] [INSPIRE].

[59] K. Funakubo, A. Kakuto and K. Takenaga, The effective potential of electroweak theory with two massless Higgs doublets at finite temperature, Prog. Theor. Phys. 91 (1994) 341 [hep-ph/9310267] [INSPIRE].

[60] A.T. Davies, C.D. froggatt, G. Jenkins and R.G. Moorhouse, Baryogenesis constraints on two Higgs doublet models, Phys. Lett. B 336 (1994) 464 [INSPIRE].

[61] J.M. Cline, K. Kainulainen and A.P. Vischer, Dynamics of two Higgs doublet CP-violation and baryogenesis at the electroweak phase transition, Phys. Rev. D 54 (1996) 2451 [hep-ph/9506284] [INSPIRE]. 
[62] L. Fromme, S.J. Huber and M. Seniuch, Baryogenesis in the two-Higgs doublet model, JHEP 11 (2006) 038 [hep-ph/0605242] [INSPIRE].

[63] J.M. Cline, K. Kainulainen and M. Trott, Electroweak baryogenesis in two Higgs doublet models and B meson anomalies, JHEP 11 (2011) 089 [arXiv:1107.3559] [INSPIRE].

[64] G.C. Dorsch, S.J. Huber and J.M. No, A strong electroweak phase transition in the 2HDM after LHC8, JHEP 10 (2013) 029 [arXiv: 1305.6610] [INSPIRE].

[65] G.C. Dorsch, S.J. Huber, T. Konstandin and J.M. No, A second Higgs doublet in the early universe: baryogenesis and gravitational waves, JCAP 05 (2017) 052 [arXiv:1611.05874] [INSPIRE].

[66] P. Basler, M. Krause, M. Mühlleitner, J. Wittbrodt and A. Wlotzka, Strong first order electroweak phase transition in the CP-conserving 2HDM revisited, JHEP 02 (2017) 121 [arXiv: 1612.04086] [INSPIRE].

[67] P. Basler, M. Mühlleitner and J. Wittbrodt, The CP-violating 2HDM in light of a strong first order electroweak phase transition and implications for Higgs pair production, JHEP 03 (2018) 061 [arXiv:1711.04097] [INSPIRE].

[68] J. Bernon, L. Bian and Y. Jiang, A new insight into the phase transition in the early universe with two Higgs doublets, JHEP 05 (2018) 151 [arXiv: 1712.08430] [INSPIRE].

[69] H.H. Patel and M.J. Ramsey-Musolf, Stepping into electroweak symmetry breaking: phase transitions and Higgs phenomenology, Phys. Rev. D 88 (2013) 035013 [arXiv:1212.5652] [INSPIRE].

[70] M. Chala, M. Ramos and M. Spannowsky, Gravitational wave and collider probes of a triplet Higgs sector with a low cutoff, Eur. Phys. J. C 79 (2019) 156 [arXiv:1812.01901] [INSPIRE].

[71] S.S. AbdusSalam and T.A. Chowdhury, Scalar representations in the light of electroweak phase transition and cold dark matter phenomenology, JCAP 05 (2014) 026 [arXiv:1310.8152] [INSPIRE].

[72] M. Chala, G. Nardini and I. Sobolev, Unified explanation for dark matter and electroweak baryogenesis with direct detection and gravitational wave signatures, Phys. Rev. D 94 (2016) 055006 [arXiv: 1605.08663] [INSPIRE].

[73] P. Basler, M. Mühlleitner and J. Müller, Electroweak phase transition in non-minimal Higgs sectors, JHEP 05 (2020) 016 [arXiv:1912.10477] [INSPIRE].

[74] C. Grojean, G. Servant and J.D. Wells, First-order electroweak phase transition in the Standard Model with a low cutoff, Phys. Rev. D 71 (2005) 036001 [hep-ph/0407019] [INSPIRE].

[75] D. Bödeker, L. Fromme, S.J. Huber and M. Seniuch, The baryon asymmetry in the Standard Model with a low cut-off, JHEP 02 (2005) 026 [hep-ph/0412366] [INSPIRE].

[76] C. Delaunay, C. Grojean and J.D. Wells, Dynamics of non-renormalizable electroweak symmetry breaking, JHEP 04 (2008) 029 [arXiv:0711.2511] [INSPIRE].

[77] B. Grinstein and M. Trott, Electroweak baryogenesis with a pseudo-Goldstone Higgs, Phys. Rev. D 78 (2008) 075022 [arXiv:0806.1971] [InSPIRE]. 
[78] F.P. Huang, Y. Wan, D.-G. Wang, Y.-F. Cai and X. Zhang, Hearing the echoes of electroweak baryogenesis with gravitational wave detectors, Phys. Rev. D 94 (2016) 041702 [arXiv: 1601.01640] [INSPIRE].

[79] R.-G. Cai, M. Sasaki and S.-J. Wang, The gravitational waves from the first-order phase transition with a dimension-six operator, JCAP 08 (2017) 004 [arXiv:1707.03001] [INSPIRE].

[80] J. de Vries, M. Postma, J. van de Vis and G. White, Electroweak baryogenesis and the Standard Model effective field theory, JHEP 01 (2018) 089 [arXiv:1710.04061] [InSPIRE].

[81] M. Chala, C. Krause and G. Nardini, Signals of the electroweak phase transition at colliders and gravitational wave observatories, JHEP 07 (2018) 062 [arXiv: 1802.02168] [INSPIRE].

[82] M. Postma and G. White, Cosmological phase transitions: is effective field theory just a toy?, JHEP 03 (2021) 280 [arXiv: 2012.03953] [INSPIRE].

[83] M.E. Shaposhnikov, Structure of the high temperature gauge ground state and electroweak production of the baryon asymmetry, Nucl. Phys. B 299 (1988) 797 [INSPIRE].

[84] G.R. Farrar and M.E. Shaposhnikov, Baryon asymmetry of the universe in the minimal Standard Model, Phys. Rev. Lett. 70 (1993) 2833 [Erratum ibid. 71 (1993) 210] [hep-ph/9305274] [INSPIRE].

[85] G.R. Farrar and M.E. Shaposhnikov, Baryon asymmetry of the universe in the standard electroweak theory, Phys. Rev. D 50 (1994) 774 [hep-ph/9305275] [INSPIRE].

[86] M.B. Gavela, P. Hernández, J. Orloff and O. Pène, Standard Model CP-violation and baryon asymmetry, Mod. Phys. Lett. A 9 (1994) 795 [hep-ph/9312215] [InSPIRE].

[87] M.B. Gavela, M. Lozano, J. Orloff and O. Pène, Standard Model CP-violation and baryon asymmetry. Part 1. Zero temperature, Nucl. Phys. B 430 (1994) 345 [hep-ph/9406288] [INSPIRE].

[88] T. Brauner, O. Taanila, A. Tranberg and A. Vuorinen, Temperature dependence of Standard Model CP-violation, Phys. Rev. Lett. 108 (2012) 041601 [arXiv:1110.6818] [INSPIRE].

[89] T. Brauner, O. Taanila, A. Tranberg and A. Vuorinen, Computing the temperature dependence of effective CP-violation in the Standard Model, JHEP 11 (2012) 076 [arXiv: 1208.5609] [INSPIRE].

[90] D. Land and E.D. Carlson, Two stage phase transition in two Higgs models, Phys. Lett. B 292 (1992) 107 [hep-ph/9208227] [INSPIRE].

[91] S. Inoue, G. Ovanesyan and M.J. Ramsey-Musolf, Two-step electroweak baryogenesis, Phys. Rev. D 93 (2016) 015013 [arXiv:1508.05404] [INSPIRE].

[92] N. Blinov, J. Kozaczuk, D.E. Morrissey and C. Tamarit, Electroweak baryogenesis from exotic electroweak symmetry breaking, Phys. Rev. D 92 (2015) 035012 [arXiv:1504.05195] [INSPIRE].

[93] D. Croon and G. White, Exotic gravitational wave signatures from simultaneous phase transitions, JHEP 05 (2018) 210 [arXiv: 1803.05438] [INSPIRE].

[94] P. Schwaller, Gravitational waves from a dark phase transition, Phys. Rev. Lett. 115 (2015) 181101 [arXiv:1504.07263] [INSPIRE].

[95] W.-C. Huang, M. Reichert, F. Sannino and Z.-W. Wang, Testing the dark confined landscape: from lattice to gravitational waves, arXiv:2012.11614 [INSPIRE]. 
[96] D. Croon, V. Sanz and G. White, Model discrimination in gravitational wave spectra from dark phase transitions, JHEP 08 (2018) 203 [arXiv: 1806.02332] [INSPIRE].

[97] D. Croon, T.E. Gonzalo and G. White, Gravitational waves from a Pati-Salam phase transition, JHEP 02 (2019) 083 [arXiv:1812.02747] [INSPIRE].

[98] W.-C. Huang, F. Sannino and Z.-W. Wang, Gravitational waves from Pati-Salam dynamics, Phys. Rev. D 102 (2020) 095025 [arXiv: 2004.02332] [InSPIRE].

[99] E. Hall, T. Konstandin, R. McGehee, H. Murayama and G. Servant, Baryogenesis from a dark first-order phase transition, JHEP 04 (2020) 042 [arXiv: 1910.08068] [INSPIRE].

[100] D. Croon, A. Kusenko, A. Mazumdar and G. White, Solitosynthesis and gravitational waves, Phys. Rev. D 101 (2020) 085010 [arXiv:1910.09562] [INSPIRE].

[101] D. Croon, R. Houtz and V. Sanz, Dynamical axions and gravitational waves, JHEP 07 (2019) 146 [arXiv: 1904.10967] [INSPIRE].

[102] A.D. Linde, Infrared problem in thermodynamics of the Yang-Mills gas, Phys. Lett. B 96 (1980) 289 [inSPIRE].

[103] E. Braaten and R.D. Pisarski, Simple effective Lagrangian for hard thermal loops, Phys. Rev. D 45 (1992) R1827 [INSPIRE].

[104] P.B. Arnold and O. Espinosa, The effective potential and first order phase transitions: beyond leading-order, Phys. Rev. D 47 (1993) 3546 [Erratum ibid. 50 (1994) 6662] [hep-ph/9212235] [INSPIRE].

[105] K. Farakos, K. Kajantie, K. Rummukainen and M.E. Shaposhnikov, 3D physics and the electroweak phase transition: a framework for lattice Monte Carlo analysis, Nucl. Phys. B 442 (1995) 317 [hep-lat/9412091] [INSPIRE].

[106] K. Kajantie, M. Laine, K. Rummukainen and M.E. Shaposhnikov, The electroweak phase transition: a nonperturbative analysis, Nucl. Phys. B 466 (1996) 189 [hep-lat/9510020] [INSPIRE].

[107] P.H. Ginsparg, First order and second order phase transitions in gauge theories at finite temperature, Nucl. Phys. B 170 (1980) 388 [INSPIRE].

[108] T. Appelquist and R.D. Pisarski, High-temperature Yang-Mills theories and three-dimensional quantum chromodynamics, Phys. Rev. D 23 (1981) 2305 [InSPIRE].

[109] S. Nadkarni, Dimensional reduction in hot QCD, Phys. Rev. D 27 (1983) 917 [INSPIRE].

[110] N.P. Landsman, Limitations to dimensional reduction at high temperature, Nucl. Phys. B 322 (1989) 498 [INSPIRE].

[111] K. Kajantie, M. Laine, K. Rummukainen and M.E. Shaposhnikov, Generic rules for high temperature dimensional reduction and their application to the Standard Model, Nucl. Phys. B 458 (1996) 90 [hep-ph/9508379] [INSPIRE].

[112] E. Braaten and A. Nieto, Effective field theory approach to high temperature thermodynamics, Phys. Rev. D 51 (1995) 6990 [hep-ph/9501375] [INSPIRE].

[113] E. Braaten and A. Nieto, Free energy of QCD at high temperature, Phys. Rev. D 53 (1996) 3421 [hep-ph/9510408] [INSPIRE].

[114] K. Kajantie, M. Laine, K. Rummukainen and M.E. Shaposhnikov, $3 D \mathrm{SU}(N)+$ adjoint Higgs theory and finite temperature QCD, Nucl. Phys. B 503 (1997) 357 [hep-ph/9704416] [INSPIRE]. 
[115] K. Kajantie, M. Laine, J. Peisa, A. Rajantie, K. Rummukainen and M.E. Shaposhnikov, Nonperturbative Debye mass in finite temperature QCD, Phys. Rev. Lett. 79 (1997) 3130 [hep-ph/9708207] [INSPIRE].

[116] J.O. Andersen, On effective field theories at finite temperature, Ph.D. thesis, University of Oslo, Oslo, Norway (1997) [hep-ph/9709331] [INSPIRE].

[117] K. Kajantie, M. Laine, A. Rajantie, K. Rummukainen and M. Tsypin, The phase diagram of three-dimensional SU(3) + adjoint Higgs theory, JHEP 11 (1998) 011 [hep-lat/9811004] [INSPIRE].

[118] K. Kajantie, M. Laine, K. Rummukainen and Y. Schröder, How to resum long distance contributions to the QCD pressure?, Phys. Rev. Lett. 86 (2001) 10 [hep-ph/0007109] [INSPIRE].

[119] K. Kajantie, M. Laine, K. Rummukainen and Y. Schröder, The pressure of hot QCD up to $g^{6} \ln (1 / g)$, Phys. Rev. D 67 (2003) 105008 [hep-ph/0211321] [INSPIRE].

[120] K. Kajantie, M. Laine, K. Rummukainen and Y. Schröder, Four loop vacuum energy density of the $\mathrm{SU}\left(N_{c}\right)+$ adjoint Higgs theory, JHEP 04 (2003) 036 [hep-ph/0304048] [INSPIRE].

[121] M. Laine and M. Vepsäläinen, Mesonic correlation lengths in high temperature QCD, JHEP 02 (2004) 004 [hep-ph/0311268] [INSPIRE].

[122] A. Hietanen, K. Kajantie, M. Laine, K. Rummukainen and Y. Schröder, Plaquette expectation value and gluon condensate in three dimensions, JHEP 01 (2005) 013 [hep-lat/0412008] [INSPIRE].

[123] M. Vepsäläinen, Mesonic screening masses at high temperature and finite density, JHEP 03 (2007) 022 [hep-ph/0701250] [INSPIRE].

[124] A. Hietanen, K. Kajantie, M. Laine, K. Rummukainen and Y. Schröder, Three-dimensional physics and the pressure of hot QCD, Phys. Rev. D 79 (2009) 045018 [arXiv:0811.4664] [INSPIRE].

[125] J. Ghiglieri, A. Kurkela, M. Strickland and A. Vuorinen, Perturbative thermal QCD: formalism and applications, Phys. Rept. 880 (2020) 1 [arXiv:2002.10188] [INSPIRE].

[126] M. Gürtler, E.-M. Ilgenfritz, J. Kripfganz, H. Perlt and A. Schiller, Three-dimensional lattice studies of the electroweak phase transition at $M_{\mathrm{Higgs}}$ approximates $70 \mathrm{GeV}$, Nucl. Phys. B 483 (1997) 383 [hep-lat/9605042] [INSPIRE].

[127] K. Kajantie, M. Laine, K. Rummukainen and M.E. Shaposhnikov, High temperature dimensional reduction and parity violation, Phys. Lett. B 423 (1998) 137 [hep-ph/9710538] [INSPIRE].

[128] K. Rummukainen, M. Tsypin, K. Kajantie, M. Laine and M.E. Shaposhnikov, The universality class of the electroweak theory, Nucl. Phys. B 532 (1998) 283 [hep-lat/9805013] [INSPIRE].

[129] A. Gynther and M. Vepsäläinen, Pressure of the Standard Model at high temperatures, JHEP 01 (2006) 060 [hep-ph/0510375] [INSPIRE].

[130] A. Gynther and M. Vepsäläinen, Pressure of the Standard Model near the electroweak phase transition, JHEP 03 (2006) 011 [hep-ph/0512177] [INSPIRE].

[131] M. Vepsäläinen, Applications of dimensional reduction to electroweak and QCD matter, Ph.D. thesis, Helsinki U., Helsinki, Finland (2007) [arXiv:0709.2773] [INSPIRE]. 
[132] M. Losada, High temperature dimensional reduction of the MSSM and other multiscalar models, Phys. Rev. D 56 (1997) 2893 [hep-ph/9605266] [INSPIRE].

[133] M. Losada, The electroweak phase transition in the minimal supersymmetric Standard Model, Ph.D. thesis, Rutgers U., Piscataway, NJ, U.S.A. (1996) [hep-ph/9612337] [INSPIRE].

[134] G.R. Farrar and M. Losada, SUSY and the electroweak phase transition, Phys. Lett. B 406 (1997) 60 [hep-ph/9612346] [INSPIRE].

[135] J.M. Cline and K. Kainulainen, Supersymmetric electroweak phase transition: beyond perturbation theory, Nucl. Phys. B 482 (1996) 73 [hep-ph/9605235] [INSPIRE].

[136] J.M. Cline and K. Kainulainen, Supersymmetric electroweak phase transition: dimensional reduction versus effective potential, Nucl. Phys. B 510 (1998) 88 [hep-ph/9705201] [INSPIRE].

[137] D. Bödeker, P. John, M. Laine and M.G. Schmidt, The two loop MSSM finite temperature effective potential with stop condensation, Nucl. Phys. B 497 (1997) 387 [hep-ph/9612364] [INSPIRE].

[138] M. Laine and K. Rummukainen, A strong electroweak phase transition up to $m_{H}$ is about $105 \mathrm{GeV}$, Phys. Rev. Lett. 80 (1998) 5259 [hep-ph/9804255] [INSPIRE].

[139] M. Laine and K. Rummukainen, The MSSM electroweak phase transition on the lattice, Nucl. Phys. B 535 (1998) 423 [hep-lat/9804019] [INSPIRE].

[140] M. Laine and K. Rummukainen, Higgs sector CP-violation at the electroweak phase transition, Nucl. Phys. B 545 (1999) 141 [hep-ph/9811369] [INSPIRE].

[141] M. Laine and M. Losada, Two loop dimensional reduction and effective potential without temperature expansions, Nucl. Phys. B 582 (2000) 277 [hep-ph/0003111] [INSPIRE].

[142] M. Laine, G. Nardini and K. Rummukainen, Lattice study of an electroweak phase transition at $m_{h} \simeq 126 \mathrm{GeV}, J C A P 01$ (2013) 011 [arXiv:1211.7344] [INSPIRE].

[143] J.O. Andersen, Dimensional reduction of the two Higgs doublet model at high temperature, Eur. Phys. J. C 11 (1999) 563 [hep-ph/9804280] [inSPIRE].

[144] M. Laine and K. Rummukainen, Two Higgs doublet dynamics at the electroweak phase transition: a nonperturbative study, Nucl. Phys. B 597 (2001) 23 [hep-lat/0009025] [INSPIRE].

[145] M. Karjalainen and J. Peisa, Dimensionally reduced U(1) + Higgs theory in the broken phase, Z. Phys. C 76 (1997) 319 [hep-lat/9607023] [INSPIRE].

[146] K. Kajantie, M. Karjalainen, M. Laine and J. Peisa, Masses and phase structure in the Ginzburg-Landau model, Phys. Rev. B 57 (1998) 3011 [cond-mat/9704056] [INSPIRE].

[147] K. Kajantie, M. Karjalainen, M. Laine and J. Peisa, Three-dimensional U(1) gauge + Higgs theory as an effective theory for finite temperature phase transitions,

Nucl. Phys. B 520 (1998) 345 [hep-lat/9711048] [INSPIRE].

[148] J.O. Andersen, 3D effective field theory for finite temperature scalar electrodynamics, Phys. Rev. D 59 (1999) 065015 [hep-ph/9709418] [INSPIRE].

[149] A. Rajantie, $\mathrm{SU}(5)+$ adjoint Higgs model at finite temperature, Nucl. Phys. B 501 (1997) 521 [hep-ph/9702255] [INSPIRE]. 
[150] K. Jansen and M. Laine, Inverse symmetry breaking with $4 D$ lattice simulations, Phys. Lett. B 435 (1998) 166 [hep-lat/9805024] [INSPIRE].

[151] T. Brauner, T.V.I. Tenkanen, A. Tranberg, A. Vuorinen and D.J. Weir, Dimensional reduction of the Standard Model coupled to a new singlet scalar field, JHEP 03 (2017) 007 [arXiv: 1609.06230] [INSPIRE].

[152] O. Gould, J. Kozaczuk, L. Niemi, M.J. Ramsey-Musolf, T.V.I. Tenkanen and D.J. Weir, Nonperturbative analysis of the gravitational waves from a first-order electroweak phase transition, Phys. Rev. D 100 (2019) 115024 [arXiv:1903.11604] [InSPIRE].

[153] L. Niemi, H.H. Patel, M.J. Ramsey-Musolf, T.V.I. Tenkanen and D.J. Weir, Electroweak phase transition in the real triplet extension of the SM: dimensional reduction, Phys. Rev. D 100 (2019) 035002 [arXiv:1802.10500] [InSPIRE].

[154] L. Niemi, M.J. Ramsey-Musolf, T.V.I. Tenkanen and D.J. Weir, Thermodynamics of a two-step electroweak phase transition, Phys. Rev. Lett. 126 (2021) 171802 [arXiv:2005.11332] [INSPIRE].

[155] A. Helset, Dimensional reduction of the two-Higgs doublet model with a softly broken $Z_{2}$ symmetry at one-loop, master's thesis, Norwegian U. Sci. Tech., Trondheim, Norway (2017).

[156] J.O. Andersen et al., Nonperturbative analysis of the electroweak phase transition in the two Higgs doublet model, Phys. Rev. Lett. 121 (2018) 191802 [arXiv:1711.09849] [INSPIRE].

[157] T. Gorda, A. Helset, L. Niemi, T.V.I. Tenkanen and D.J. Weir, Three-dimensional effective theories for the two Higgs doublet model at high temperature, JHEP 02 (2019) 081 [arXiv: 1802.05056] [INSPIRE].

[158] K. Kainulainen, V. Keus, L. Niemi, K. Rummukainen, T.V.I. Tenkanen and V. Vaskonen, On the validity of perturbative studies of the electroweak phase transition in the two Higgs doublet model, JHEP 06 (2019) 075 [arXiv: 1904.01329] [INSPIRE].

[159] D. Croon, O. Gould, P. Schicho, T.V.I. Tenkanen and G. White, Theoretical uncertainties for cosmological first-order phase transitions, JHEP 04 (2021) 055 [arXiv: 2009.10080] [INSPIRE].

[160] O. Gould, Real scalar phase transitions: a nonperturbative analysis, JHEP 04 (2021) 057 [arXiv: 2101.05528] [INSPIRE].

[161] L. Dolan and R. Jackiw, Symmetry behavior at finite temperature, Phys. Rev. D 9 (1974) 3320 [InSPIRE].

[162] M.E. Carrington, The effective potential at finite temperature in the Standard Model, Phys. Rev. D 45 (1992) 2933 [InSPIRE].

[163] R.R. Parwani, Resummation in a hot scalar field theory, Phys. Rev. D 45 (1992) 4695 [Erratum ibid. 48 (1993) 5965] [hep-ph/9204216] [INSPIRE].

[164] W. Buchmüller, Z. Fodor, T. Helbig and D. Walliser, The weak electroweak phase transition, Annals Phys. 234 (1994) 260 [hep-ph/9303251] [INSPIRE].

[165] Z. Fodor and A. Hebecker, Finite temperature effective potential to order $g^{4}, \lambda^{2}$ and the electroweak phase transition, Nucl. Phys. B 432 (1994) 127 [hep-ph/9403219] [INSPIRE].

[166] J. Kripfganz, A. Laser and M.G. Schmidt, The high temperature two loop effective potential of the electroweak theory in a general 't Hooft background gauge, Phys. Lett. B 351 (1995) 266 [hep-ph/9501317] [INSPIRE]. 
[167] M. Laine, M. Meyer and G. Nardini, Thermal phase transition with full 2-loop effective potential, Nucl. Phys. B 920 (2017) 565 [arXiv:1702.07479] [InSPIRE].

[168] A. Ekstedt and J. Löfgren, A critical look at the electroweak phase transition, JHEP 12 (2020) 136 [arXiv:2006.12614] [INSPIRE].

[169] K. Farakos, K. Kajantie, K. Rummukainen and M.E. Shaposhnikov, 3D physics and the electroweak phase transition: perturbation theory, Nucl. Phys. B 425 (1994) 67 [hep-ph/9404201] [INSPIRE].

[170] M. Laine, The two loop effective potential of the $3 D \mathrm{SU}(2)$ Higgs model in a general covariant gauge, Phys. Lett. B 335 (1994) 173 [hep-ph/9406268] [INSPIRE].

[171] P. Schicho, Multi-loop investigations of strong interactions at high temperatures, Ph.D. thesis, U. Bern, Bern, Switzerland (2020).

[172] L. Niemi, P. Schicho and T.V.I. Tenkanen, Singlet-assisted electroweak phase transition at two loops, arXiv: 2103.07467 [INSPIRE].

[173] O. Gould and T.V.I. Tenkanen, On renormalisation scale dependence of the effective potential at high temperature, forthcoming, (2021).

[174] M. Lüscher, Chiral gauge theories revisited, Subnucl. Ser. 38 (2002) 41 [hep-th/0102028] [INSPIRE].

[175] M. Laine, Comparison of $4 D$ and $3 D$ lattice results for the electroweak phase transition, Phys. Lett. B 385 (1996) 249 [hep-lat/9604011] [INSPIRE].

[176] C. Caprini et al., Detecting gravitational waves from cosmological phase transitions with LISA: an update, JCAP 03 (2020) 024 [arXiv:1910.13125] [INSPIRE].

[177] K. Schmitz, LISA sensitivity to gravitational waves from sound waves, Symmetry 12 (2020) 1477 [arXiv: 2005.10789] [INSPIRE].

[178] B.M. Dillon, B.K. El-Menoufi, S.J. Huber and J.P. Manuel, Rapid holographic phase transition with brane-localized curvature, Phys. Rev. D 98 (2018) 086005 [arXiv: 1708.02953] [INSPIRE].

[179] E. Megías, G. Nardini and M. Quirós, Cosmological phase transitions in warped space: gravitational waves and collider signatures, JHEP 09 (2018) 095 [arXiv:1806.04877] [INSPIRE].

[180] F.R. Ares, M. Hindmarsh, C. Hoyos and N. Jokela, Gravitational waves from a holographic phase transition, JHEP 04 (2021) 100 [arXiv:2011.12878] [INSPIRE].

[181] J.R. Espinosa, B. Gripaios, T. Konstandin and F. Riva, Electroweak baryogenesis in non-minimal composite Higgs models, JCAP 01 (2012) 012 [arXiv:1110.2876] [INSPIRE].

[182] S. Bruggisser, B. Von Harling, O. Matsedonskyi and G. Servant, Electroweak phase transition and baryogenesis in composite Higgs models, JHEP 12 (2018) 099 [arXiv: 1804.07314] [INSPIRE].

[183] L. Bian, Y. Wu and K.-P. Xie, Electroweak phase transition with composite Higgs models: calculability, gravitational waves and collider searches, JHEP 12 (2019) 028 [arXiv:1909.02014] [INSPIRE].

[184] A. Katz and M. Perelstein, Higgs couplings and electroweak phase transition, JHEP 07 (2014) 108 [arXiv: 1401.1827] [INSPIRE].

[185] J.S. Langer, Theory of the condensation point, Annals Phys. 41 (1967) 108 [INSPIRE]. 
[186] J.S. Langer, Statistical theory of the decay of metastable states, Annals Phys. 54 (1969) 258 [INSPIRE].

[187] S.R. Coleman, The fate of the false vacuum. 1. Semiclassical theory, Phys. Rev. D 15 (1977) 2929 [Erratum ibid. 16 (1977) 1248] [InSPIRE].

[188] A.D. Linde, Decay of the false vacuum at finite temperature, Nucl. Phys. B 216 (1983) 421 [Erratum ibid. 223 (1983) 544] [INSPIRE].

[189] A. Kosowsky, M.S. Turner and R. Watkins, Gravitational waves from first order cosmological phase transitions, Phys. Rev. Lett. 69 (1992) 2026 [INSPIRE].

[190] M. Kamionkowski, A. Kosowsky and M.S. Turner, Gravitational radiation from first order phase transitions, Phys. Rev. D 49 (1994) 2837 [astro-ph/9310044] [INSPIRE].

[191] J. Ignatius, K. Kajantie, H. Kurki-Suonio and M. Laine, The growth of bubbles in cosmological phase transitions, Phys. Rev. D 49 (1994) 3854 [astro-ph/9309059] [INSPIRE].

[192] H. Kurki-Suonio and M. Laine, Supersonic deflagrations in cosmological phase transitions, Phys. Rev. D 51 (1995) 5431 [hep-ph/9501216] [InSPIRE].

[193] S.J. Huber and T. Konstandin, Gravitational wave production by collisions: more bubbles, JCAP 09 (2008) 022 [arXiv:0806.1828] [inSPIRE].

[194] F. Giese, T. Konstandin and J. van de Vis, Model-independent energy budget of cosmological first-order phase transitions - a sound argument to go beyond the bag model, JCAP 07 (2020) 057 [arXiv: 2004.06995] [INSPIRE].

[195] D. Bödeker and G.D. Moore, Can electroweak bubble walls run away?, JCAP 05 (2009) 009 [arXiv: 0903.4099] [INSPIRE].

[196] J. Kozaczuk, Bubble expansion and the viability of singlet-driven electroweak baryogenesis, JHEP 10 (2015) 135 [arXiv: 1506.04741] [INSPIRE].

[197] D. Bödeker and G.D. Moore, Electroweak bubble wall speed limit, JCAP 05 (2017) 025 [arXiv: 1703.08215] [INSPIRE].

[198] G.C. Dorsch, S.J. Huber and T. Konstandin, Bubble wall velocities in the Standard Model and beyond, JCAP 12 (2018) 034 [arXiv: 1809.04907] [INSPIRE].

[199] S. Höche, J. Kozaczuk, A.J. Long, J. Turner and Y. Wang, Towards an all-orders calculation of the electroweak bubble wall velocity, JCAP 03 (2021) 009 [arXiv:2007.10343] [INSPIRE].

[200] R.-G. Cai and S.-J. Wang, Effective picture of bubble expansion, JCAP 03 (2021) 096 [arXiv:2011.11451] [INSPIRE].

[201] G.D. Moore and K. Rummukainen, Electroweak bubble nucleation, nonperturbatively, Phys. Rev. D 63 (2001) 045002 [hep-ph/0009132] [INSPIRE].

[202] G.D. Moore, K. Rummukainen and A. Tranberg, Nonperturbative computation of the bubble nucleation rate in the cubic anisotropy model, JHEP 04 (2001) 017 [hep-lat/0103036] [INSPIRE].

[203] M. Hindmarsh, S.J. Huber, K. Rummukainen and D.J. Weir, Gravitational waves from the sound of a first order phase transition, Phys. Rev. Lett. 112 (2014) 041301 [arXiv: 1304.2433] [INSPIRE]. 
[204] M. Hindmarsh, S.J. Huber, K. Rummukainen and D.J. Weir, Numerical simulations of acoustically generated gravitational waves at a first order phase transition, Phys. Rev. D 92 (2015) 123009 [arXiv:1504.03291] [InSPIRE].

[205] M. Hindmarsh, S.J. Huber, K. Rummukainen and D.J. Weir, Shape of the acoustic gravitational wave power spectrum from a first order phase transition, Phys. Rev. D 96 (2017) 103520 [Erratum ibid. 101 (2020) 089902] [arXiv:1704.05871] [INSPIRE].

[206] D. Cutting, M. Hindmarsh and D.J. Weir, Gravitational waves from vacuum first-order phase transitions: from the envelope to the lattice, Phys. Rev. D 97 (2018) 123513 [arXiv: 1802.05712] [INSPIRE].

[207] D. Cutting, M. Hindmarsh and D.J. Weir, Vorticity, kinetic energy, and suppressed gravitational wave production in strong first order phase transitions, Phys. Rev. Lett. 125 (2020) 021302 [arXiv: 1906.00480] [INSPIRE].

[208] D. Cutting, E.G. Escartin, M. Hindmarsh and D.J. Weir, Gravitational waves from vacuum first order phase transitions II: from thin to thick walls, Phys. Rev. D 103 (2021) 023531 [arXiv: 2005.13537] [INSPIRE].

[209] G.A. White, A pedagogical introduction to electroweak baryogenesis, Morgan \& Claypool Publishers, (2016) [INSPIRE].

[210] M. Laine, $3 D$ effective theories for the Standard Model and extensions, in $2^{\text {nd }}$ international conference on strong and electroweak matter, (1997), pg. 160 [hep-ph/9707415] [INSPIRE].

[211] J.O. Andersen and M. Strickland, Resummation in hot field theories, Annals Phys. 317 (2005) 281 [hep-ph/0404164] [INSPIRE].

[212] M. Laine and A. Vuorinen, Basics of thermal field theory, Lect. Notes Phys. 925 (2016) 1 [arXiv: 1701.01554] [INSPIRE].

[213] T. Matsubara, A new approach to quantum statistical mechanics, Prog. Theor. Phys. 14 (1955) 351 [inSPIRE].

[214] P. Basler and M. Mühlleitner, BSMPT (Beyond the Standard Model Phase Transitions): a tool for the electroweak phase transition in extended Higgs sectors, Comput. Phys. Commun. 237 (2019) 62 [arXiv:1803.02846] [INSPIRE].

[215] C.L. Wainwright, CosmoTransitions: computing cosmological phase transition temperatures and bubble profiles with multiple fields, Comput. Phys. Commun. 183 (2012) 2006 [arXiv:1109.4189] [INSPIRE].

[216] P. Athron, C. Balázs, A. Fowlie and Y. Zhang, PhaseTracer: tracing cosmological phases and calculating transition properties, Eur. Phys. J. C 80 (2020) 567 [arXiv:2003.02859] [INSPIRE].

[217] H.H. Patel and M.J. Ramsey-Musolf, Baryon washout, electroweak phase transition, and perturbation theory, JHEP 07 (2011) 029 [arXiv:1101.4665] [INSPIRE].

[218] M. Laine, Gauge dependence of the high temperature two loop effective potential for the Higgs field, Phys. Rev. D 51 (1995) 4525 [hep-ph/9411252] [INSPIRE].

[219] A.K. Rajantie, Feynman diagrams to three loops in three-dimensional field theory, Nucl. Phys. B 480 (1996) 729 [Erratum ibid. 513 (1998) 761] [hep-ph/9606216] [INSPIRE]. 
[220] M. Laine, Exact relation of lattice and continuum parameters in three-dimensional SU(2) + Higgs theories, Nucl. Phys. B 451 (1995) 484 [hep-lat/9504001] [INSPIRE].

[221] M. Laine and A. Rajantie, Lattice continuum relations for $3 D \mathrm{SU}(N)+$ Higgs theories, Nucl. Phys. B 513 (1998) 471 [hep-lat/9705003] [INSPIRE].

[222] H.J. Rothe, Lattice gauge theories: an introduction, World Sci. Lect. Notes Phys. 43 (1992) 1.

[223] P. Athron, C. Balázs, M. Bardsley, A. Fowlie, D. Harries and G. White, BubbleProfiler: finding the field profile and action for cosmological phase transitions, Comput. Phys. Commun. 244 (2019) 448 [arXiv: 1901.03714] [InSPIRE].

[224] M. Nishimura and Y. Schröder, IBP methods at finite temperature, JHEP 09 (2012) 051 [arXiv: 1207.4042] [INSPIRE].

[225] M. Laine, P. Schicho and Y. Schröder, Soft thermal contributions to 3-loop gauge coupling, JHEP 05 (2018) 037 [arXiv: 1803.08689] [INSPIRE].

[226] G.D. Moore, Measuring the broken phase sphaleron rate nonperturbatively, Phys. Rev. D 59 (1999) 014503 [hep-ph/9805264] [INSPIRE].

[227] B. Ruijl, T. Ueda and J. Vermaseren, FORM version 4.2, arXiv:1707.06453 [InSPIRE].

[228] P. Nogueira, Automatic Feynman graph generation, J. Comput. Phys. 105 (1993) 279.

[229] S. Laporta, High precision calculation of multiloop Feynman integrals by difference equations, Int. J. Mod. Phys. A 15 (2000) 5087 [hep-ph/0102033] [inSPIRE].

[230] P.B. Arnold and C.-X. Zhai, The three loop free energy for high temperature QED and QCD with fermions, Phys. Rev. D 51 (1995) 1906 [hep-ph/9410360] [INSPIRE].

[231] J. Österman, Evaluation of master integrals in thermal field theory, master's thesis, University of Helsinki, Helsinki, Finland (2019).

[232] E.E. Jenkins, A.V. Manohar and M. Trott, Renormalization group evolution of the Standard Model dimension six operators. Part I. Formalism and $\lambda$ dependence, JHEP 10 (2013) 087 [arXiv: 1308.2627] [INSPIRE].

[233] J.C. Collins and J.A.M. Vermaseren, Axodraw version 2, arXiv:1606.01177 [INSPIRE].

[234] E.E. Jenkins, A.V. Manohar and M. Trott, Renormalization group evolution of the Standard Model dimension six operators. Part II. Yukawa dependence, JHEP 01 (2014) 035 [arXiv:1310.4838] [INSPIRE].

[235] R. Alonso, E.E. Jenkins, A.V. Manohar and M. Trott, Renormalization group evolution of the Standard Model dimension six operators. Part III. Gauge coupling dependence and phenomenology, JHEP 04 (2014) 159 [arXiv:1312.2014] [INSPIRE].

[236] A. Andreassen, Gauge dependence of the quantum field theory effective potential, master's thesis, Norwegian U. Sci. Tech., Trondheim, Norway (2013).

[237] A. Ekstedt and J. Löfgren, The high-temperature expansion of the thermal sunset, arXiv: 2006.02179 [INSPIRE].

[238] P.B. Arnold and C.-X. Zhai, The three loop free energy for pure gauge QCD, Phys. Rev. D 50 (1994) 7603 [hep-ph/9408276] [InSPIRE]. 